\section{EVALUACIÓN DE LA}

TRANSFERENCIA DE CALOR EN

ESTADO ESTACIONARIOY

DINÁMICO DE UN LECHO

QUASI-ADIABÁTICO Y CON

CALENTAMIENTO EN LA PARED

CON UNA BAJA RELACIÓN DE

$d_{d} / d_{p}$
En la Ciudad de México, se presentaron a las 11:00 horas del día 29 del mes de agosto del año 2017 en la Unidad Iztapalapa de la Universidad Autónoma Metropolitana, los suscritos miembros del jurado:

DR. RICHARD STEVE RUIZ MARTINEZ

DR. ELISEO HERNÁNDEZ MARTÍNEZ

DR. CARLOS OMAR CASTILLO ARAIZA

Bajo la Presidencia del primero y con carácter de Secretario el último, se reunieron para proceder al Examen de Grado cuya denominación aparece al margen, para la obtención del grado de:

MAESTRO EN CIENCIAS (INGENIERIA QUIMICA)

DE: ALEJANDRO ROMERO LIMONES

y de acuerdo con el artículo 78 fracción III del Reglamento de Estudios Superiores de la Universidad Autónoma Metropolitana, los miembros del jurado resolvieron:

\title{
APROBAR
}

Acto continuo, el presidente del jurado comunicó al interesado el resultado de la evaluación $y$, en caso aprobatorio, le fue tomada la protesta.

LIC. JULIO CES LADA LARA ISASSI

DIRECTOR DE SIS KENASAS ESCQLARES

(1)
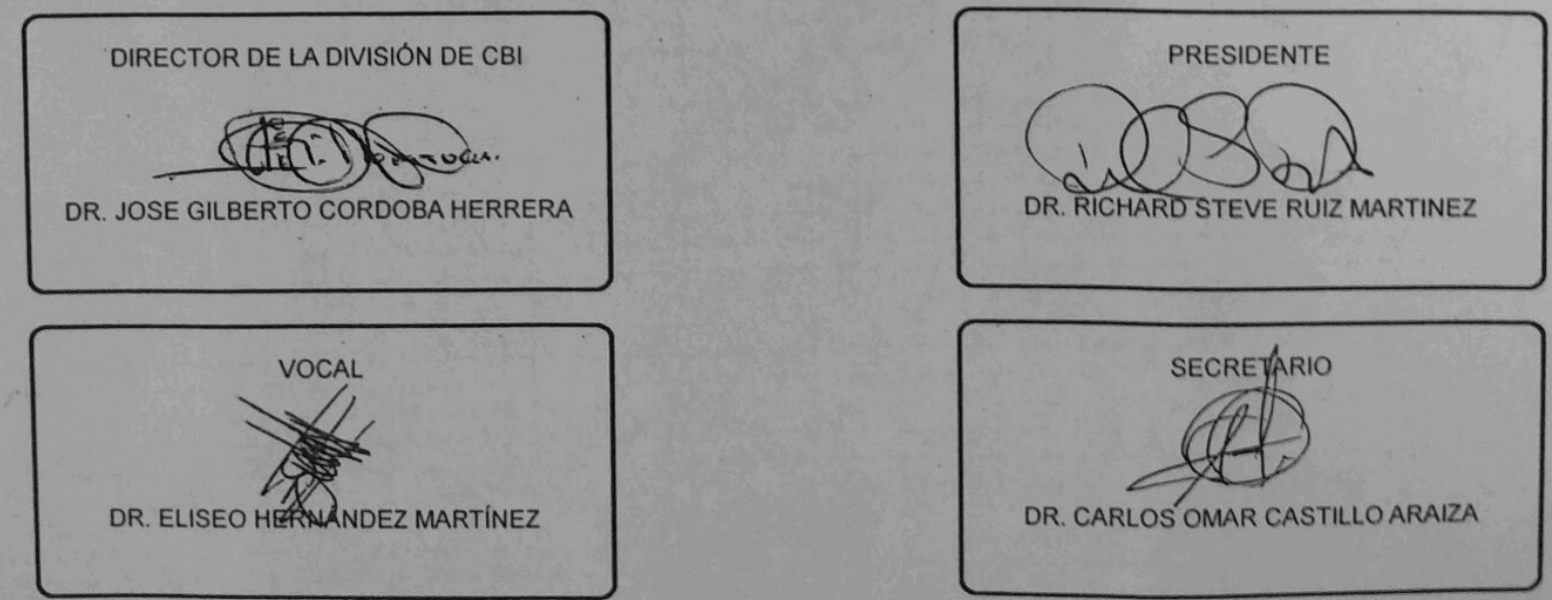
“EVALUACIÓN DE LA TRANSFERENCIA DE

CALOR EN ESTADO ESTACIONARIO Y

DINÁMICO DE UN LECHO QUASI-ADIABÁTICO

Y CON CALENTAMIENTO EN LA PARED CON

UNA BAJA RELACIÓN DE $\mathrm{d}_{\mathrm{t}} / \mathrm{d}_{\mathrm{p}}$ "

TESIS PARA OBTENER EL GRADO DE MAESTRO EN CIENCIAS EN INGENIERIA QUÍMICA

PRESENTA:

I. Q. ALEJANDRO ROMERO LIMONES

ASESOR:

Dr. CARLOS OMAR CASTILLO ARAIZA

SINODALES:

Dr. RICHARD STEVE RUIZ MARTÍNEZ

Dr. ELISEO HERNÁNDEZ MARTÍNEZ

AGOSTO 2017 


\section{Agradecimientos}

Agradezco y dedico principalmente este trabajo a mi papá y a mi mamá, Alejandro y Sandra. Estoy aquí gracias a sus sacrificios y a su apoyo incondicional. Este trabajo también es de ustedes.

A mis compañeros y amigos de la UAM-I, quienes trabajan en el grupo de investigación, quienes han estado desde que empezó la maestría, y sobre todo a aquellos con quienes realizamos convivios y actividades deportivas, gracias por hacer muy agradable el ambiente en el posgrado.

A mi asesor, Omar, y a Gus, por sus observaciones y enseñanzas en este proceso formativo llamado maestría.

A CONACyT por el apoyo económico brindado para poder realizar mi proyecto de investigación de maestría.

Y a Gaby, porque simplemente vives mejor que nadie el día a día conmigo. Estás ahí para apoyarme cuando las cosas están muy difíciles y también compartes las alegrías cuando nos toca celebrar, pero sobre todo, gracias porque contigo no sólo he crecido como profesionista sino también como persona. 


\section{Resumen General}

En la búsqueda de nuevos procesos que tengan un bajo impacto ambiental y energético para la producción de etileno, se ha propuesto la deshidrogenación oxidativa de etano a etileno (DHO). No obstante, en la actualidad no se tiene una unidad industrial para llevar a cabo esta oxidación selectiva. El reactor de lecho empacado con baja relación diámetro de tubo diámetro de partícula $\left(\mathrm{d}_{\mathrm{t}} / \mathrm{d}_{\mathrm{p}}<10\right)$ se ha propuesto como la principal tecnología para llevar a cabo esta reacción. En este tipo de reactores también se llevan a cabo una amplia gama de oxidaciones selectivas de hidrocarburos, como la oxidación parcial de o-xileno a anhídrido ftálico, la oxidación de etileno a óxido de etileno, entre otras.

A pesar de que este tipo de reacciones han sido estudiadas desde el principio del siglo XX, hoy en día no existe un modelo generalizado capaz de describir el comportamiento de estas reacciones en un reactor industrial de lecho empacado con calentamiento en la pared. En la literatura, lo anterior se le atribuye a la mala caracterización de los mecanismos de transferencia de calor y reacción, así como también a la no consideración de perfiles de fracción vacía y velocidad, los cuales interactúan de manera compleja con los mecanismos mencionados.

Diversos autores en la literatura señalan que para tener un entendimiento sobre la transferencia de calor en un sistema es indispensable conocer los parámetros que caracterizan sus mecanismos: conductividad térmica, coeficiente de transferencia de calor y/o el coeficiente de transferencia de calor global, según sea el caso. Para la determinación de los parámetros de transferencia de calor en un reactor industrial de lecho empacado con baja relación $\mathrm{d}_{\mathrm{t}} / \mathrm{d}_{\mathrm{p}}$, la metodología convencional es estudiar el transporte de calor en ausencia de reacción en sistemas experimentales que manejan condiciones de operación y características geométricas similares a las del reactor industrial. Sin embargo, en la literatura existen grandes diferencias y poca certidumbre sobre la obtención de dichos parámetros debido a que se han obtenido mediante diferentes condiciones que implican diferentes sistemas, geometrías, materiales, número de Reynolds de partícula y relación $\mathrm{d}_{\mathrm{t}} / \mathrm{d}_{\mathrm{p}}$, entre otras.

El objetivo de este trabajo es caracterizar la transferencia de calor en un lecho empacado en ausencia de reacción, considerando la hidrodinámica, para posteriormente emplear esta información en el diseño conceptual de un reactor industrial de lecho empacado donde se 
tenga lugar una oxidación selectiva. Implícitamente, dentro de este objetivo general, se tienen objetivos particulares como la estimación de parámetros efectivos de la transferencia de calor en estado estacionario y transitorio.

Primero se estudió el clásico sistema de un lecho empacado quasi-adiabático en donde se caracterizó la transferencia de calor axial mediante la estimación de la conductividad térmica efectiva axial. Después se estudió el sistema de un lecho empacado con calentamiento en la pared para caracterizar la transferencia de calor axial y radial mediante la estimación de la conductividad térmica efectiva radial y el coeficiente de transferencia de calor de pared.

El papel de la hidrodinámica y los gradientes de temperatura en la transferencia de calor se evaluaron en este lecho. Las observaciones experimentales de temperatura en estado transitorio y estacionario, con las cuales se validaron las distintas aproximaciones propuestas en este trabajo, se obtuvieron de trabajos anteriores dentro del grupo de investigación.

Las contribuciones principales de este trabajo se pueden resumir de la siguiente manera:

- Se elucida la importancia de la contribución sólida (estática) sobre la transferencia de calor, esencialmente cuando el lecho adiabático o no adiabático opera en estado transitorio.

- La hidrodinámica influye significativamente en los mecanismos radiales de transferencia de calor.

- Se desarrollaron correlaciones para evaluar los parámetros de transferencia de calor tomando en cuenta las contribuciones estática y dinámica.

- Se hace notar la diferencia de los valores de las keff,r utilizando diferentes aproximaciones. 
Índice

$\begin{array}{ll}\text { Resumen General } & \text { iii }\end{array}$

Índice $\quad \mathrm{V}$

Índice de Figuras $\quad$ vii

Índice de Tablas $\quad$ xi

Capítulo I - Introducción General 1

$\begin{array}{ll}\text { Objetivos } & 4\end{array}$

Capítulo II - Transferencia de calor lechos empacados 5

2.1 Generalidades 5

2.2 Transporte de calor axial $\quad 8$

2.3 Transporte de calor radial $\quad 8$

2.4 Limitantes históricas para la caracterización de la transferencia de calor en $\quad 11$ lechos empacados

2.5 Estudios realizados en lechos empacados 16

Capítulo III - Estudio de la transferencia de calor en el lecho empacado 24

3.1 Sistema experimental 24

3.1.1 Lecho quasi-adiabático 24

3.1.2 Lecho no isotérmico y no adiabático 25

3.2 Experimentos de transferencia de calor 26

3.2.1 Caso 1 - Estados estacionarios en el lecho quasi-adiabático 26

3.2.2 Caso 2 - Estados transitorios en el lecho quasi-adiabático, 27 experimento dinámico

3.2.3 Caso 3 - Estados estacionarios en el lecho no isotérmico no adiabático. 28

3.3 Modelos de transferencia de calor en el lecho empacado 28

3.3.1 Modelo adiabático pseudo-homogéneo en estado estacionario 28 
3.3.2 Modelo adiabático pseudo-homogéneo en estado transitorio

3.3.3 Modelo adiabático pseudo-heterogéneo en estado transitorio

3.3.4 Modelo no isotérmico no adiabático pseudo-homogéneo en estado transitorio

3.4 Modelo de Hidrodinámica en el lecho empacado

Capítulo IV - Resultados y discusión 34

4.1 Lecho quasi-adiabático $\quad 34$

4.1.1 Parámetros térmicos efectivos axiales en estado estacionario 34

4.1.2 Parámetros térmicos efectivos axiales en estado transitorio 39

4.1.3 Transferencia de calor interfacial y capacidad térmica específica 43

4.2 Lecho no adiabático no isotérmico 48

4.2.1 Aproximación clásica $\quad 48$

4.2.2 Aproximación con hidrodinámica $\quad 50$

4.2.3 Aproximación considerando $\mathrm{v}_{\mathrm{z}} \mathrm{y} \mathrm{v}_{\mathrm{r}}$

4.2.4 Velocidad radial 10\% de la velocidad axial, estimación con datos del 61 centro del lecho

4.2.5 Comparaciones entre las aproximaciones 63

4.2.6 Evaluación de la transferencia de calor a $\operatorname{Re}_{\mathrm{p}}=40.83$ y $81.67 \quad 67$

$\begin{array}{ll}\text { 4.2.7 Correlación de keff,r } & 70\end{array}$

$\begin{array}{ll}\text { Capítulo V - Conclusiones } & 71\end{array}$

$\begin{array}{ll}\text { Referencias Bibliográficas } & 76\end{array}$

$\begin{array}{ll}\text { Nomenclatura } & 82\end{array}$ 


\section{Índice de Figuras}

Figura 2.1. Mecanismos de transporte de calor que engloba la conductividad térmica 7 efectiva, $k_{\text {eff,z. }}$.

Figura 2.2. Correlaciones para obtener el parámetro $k_{\mathrm{eff}, \mathrm{z}}$ a diferentes $\mathrm{Re}_{\mathrm{p}}$. 8

Figura 2.3. Correlaciones para obtener el parámetro $h_{w}$ a diferentes $R_{p}$. 10

Figura 2.4. Correlaciones para obtener el parámetro $\mathrm{k}_{\mathrm{eff}, \mathrm{r}}$ a diferentes $\mathrm{Re}_{\mathrm{p} .} \quad 10$

Figura 2.5. Superficie de error tridimensional $\left(\mathrm{F}, \mathrm{h}_{\mathrm{w}} \mathrm{y} \mathrm{k}_{\mathrm{eff}, \mathrm{r}}\right)$ en un lecho empacado. 11

Figura 2.6. Perfiles de temperatura en un experimento con reacción de oxidación 12 de o-Xileno a $\operatorname{Re}_{\mathrm{p}}=630, \mathrm{~T}_{\text {entrada }}=463, \mathrm{~T}_{\text {baño }}=665 \mathrm{~K}$ : flujo pistón $\mathbf{\square}$, hidrodinámica $\Delta$ (componente axial), datos experimentales $\bullet$.

Figura 2.7. Perfiles de velocidad axial de aire dentro de un lecho empacado con relación $\mathrm{d}_{\mathrm{t}} / \mathrm{d}_{\mathrm{p}}=3.175, \mathrm{Re}_{\mathrm{p}}=163.36$, considerando la hidrodinámica (componente axial) $(\bullet)$, considerando flujo pistón $(-)$.

Figura 2.8. Perfil de temperatura de 2 experimentos de oxidación de o-Xileno a $\left.\operatorname{Re}_{\mathrm{p}}=630, \mathrm{~T}_{0}=463 \mathrm{~K}, \mathrm{~T}_{\mathrm{b}}=665 \mathrm{~K}(\bullet), \mathrm{T}_{\mathrm{b}}=649 \mathrm{~K}(\boldsymbol{\Delta}), \mathrm{b}\right)$ Perfiles de temperatura en un lecho adiabático.

Figura 2.9. Propuestas de distribución de velocidad axial en función del radio, Lerou y Froment ${ }^{50}$

Figura 2.10. Perfil de temperaturas en un lecho empacado, modelo que considera la hidrodinámica (---), modelo con flujo pistón (-).

Figura 3.1. Sistema experimental, lecho quasi-adiabático.

Figura 3.2. Sistema experiemental, lecho no isotérmico no adiabático

Figura 3.3. Ilustración de la región central y de pared en un lecho empacado ${ }^{3}$.

Figura 4.1. Perfiles axiales de temperatura a diferentes $\mathrm{Re}_{\mathrm{p}}$ en el lecho quasi-adiabático, modelo pseudo-homogéneo, el sombreado indica el $\pm 2.5 \mathrm{~K}$ de error.

Figura 4.2. Perfiles axiales de temperatura a diferentes $\mathrm{Re}_{\mathrm{p}}$ en el lecho quasi-adiabático, modelo pseudo-homogéneo, distintas correlaciones, el sombreado indica el $\pm 2.5 \mathrm{~K}$ de error.

Figura 4.3. Perfiles axiales de temperatura a diferentes $\mathrm{Re}_{\mathrm{p}}$ en el lecho quasi-adiabático, modelo pseudo-heterogéneo, fase sólida (-) y fase fluida (---), región sombreada representan $\operatorname{los} \pm 2.5 \mathrm{~K}$ de error. 
Figura 4.4. Perfil dinámico de temperatura en $\mathrm{z}=0 \mathrm{~cm}$ y $5 \mathrm{~cm}$ en el lecho

quasi-adiabático, modelo pseudo-homogéneo (-), dato experimental

$(\Delta)$, la región sombreada representan los $\pm 2.5 \mathrm{~K}$ de error experimental.

Figura 4.5. Perfil dinámico de temperatura en $\mathrm{z}=0 \mathrm{~cm}$ y $5 \mathrm{~cm}$ en el lecho quasi-adiabático, modelo pseudo-heterogéneo (-), la región sombreada representan los $\pm 2.5 \mathrm{~K}$ de error experimental.

Figura 4.6. Perfil dinámico de temperatura a $R_{p}=288.60$ con diferentes $h_{g}$ en $z=044$ cm en el lecho quasi-adiabático, modelo pseudo-heterogéneo, fase sólida.

Figura 4.7. Perfil dinámico de temperatura a $R_{p}=288.60$ con diferentes $h_{g}$ en $z=045$ $\mathrm{cm}$ en el lecho quasi-adiabático, modelo pseudo-heterogéneo, fase fluida.

Figura 4.8. Perfil dinámico de temperatura a $R_{p}=288.60$ con diferentes $h_{g}$ en $z=045$ $\mathrm{cm}$ en el lecho quasi-adiabático, temperatura promedio entre ambas fases.

Figura 4.9. Perfil dinámico de temperatura a $\mathrm{Re}_{\mathrm{p}}=288.60$ con diferentes $\mathrm{Cp}_{\mathrm{s}}$, en $\mathrm{z}=0 \mathrm{~cm}$ en el lecho quasi-adiabático, fase sólida.

Figura 4.10. Perfil dinámico de temperatura a $\operatorname{Re}_{\mathrm{p}}=288.60$ con diferentes $\mathrm{Cp}_{\mathrm{s}}$, en $\mathrm{z}=0 \mathrm{~cm}$ en el lecho quasi-adiabático, fase fluida.

Figura 4.11. Perfil axial de temperatura en el lecho no isotérmico no adiabático utilizando la aproximación clásica con un $\mathrm{Re}_{\mathrm{p}}=163.36$ a 3 posiciones radiales diferentes; $(\boldsymbol{\Delta})$ dato experimental, $(-)$ modelo.

Figura 4.12. Perfil radial de temperatura en el lecho no isotérmico no adiabático utilizando la aproximación clásica con un $\operatorname{Re}_{\mathrm{p}}=163.36$ a 7 posiciones axiales diferentes; $(\bullet)$ dato experimental, $(-)$ modelo.

Figura 4.13. Perfil axial de temperatura en el lecho no isotérmico no adiabático con hidrodinámica considerando $\mathrm{h}_{\mathrm{w}}$ a $\mathrm{Re}_{\mathrm{p}}=163.36,3$ posiciones radiales diferentes; $(\bullet)$ dato experimental, (-) modelo.

Figura 4.14. Perfil radial de temperatura en el lecho no isotérmico no adiabático con hidrodinámica considerando $h_{w}$ a $\operatorname{Re}_{p}=163.36,7$ posiciones axiales diferentes; $(\boldsymbol{\Delta})$ dato experimental, (-) modelo.

Figura 4.15. Perfil axial de temperatura en el lecho no isotérmico no adiabático con aproximación de la capa límite a $\mathrm{Re}_{\mathrm{p}}=163.36,3$ posiciones radiales diferentes; ( $\boldsymbol{\Delta})$ dato experimental, (-) modelo.

Figura 4.16. Perfil radial de temperatura en el lecho no isotérmico no adiabático 54 con aproximación de la capa límite a $\mathrm{Re}_{\mathrm{p}}=163.36,7$ posiciones axiales diferentes; $(\boldsymbol{\Delta})$ dato experimental, $(-)$ modelo. 
Figura 4.17. Perfil axial de temperatura en el lecho no isotérmico no adiabático con aproximación de la capa límite y empleando el $10 \%$ de $\mathrm{v}_{\mathrm{z}}$ en $\mathrm{v}_{\mathrm{r}}$, $\mathrm{Re}_{\mathrm{p}}=163.36$, a 3 posiciones radiales diferentes; $(\boldsymbol{\Delta})$ dato experimental, (一) modelo.

Figura 4.18. Perfil radial de temperatura en el lecho no isotérmico no adiabático con aproximación de la capa límite y empleando el $10 \%$ de $\mathrm{v}_{\mathrm{z}}$ en $\mathrm{v}_{\mathrm{r}}$, $\mathrm{Re}_{\mathrm{p}}=163.36$, a 7 posiciones axiales diferentes; $(\boldsymbol{\Delta})$ dato experimental, (一) modelo.

Figura 4.19. Perfil axial de temperatura en el lecho no isotérmico no adiabático con aproximación de la capa límite y empleando el $15 \%$ de $\mathrm{v}_{\mathrm{z}}$ en $\mathrm{v}_{\mathrm{r}}$, $\mathrm{Re}_{\mathrm{p}}=163.36$, a 3 posiciones radiales diferentes; $(\boldsymbol{\Delta})$ dato experimental, (一) modelo.

Figura 4.20. Perfil radial de temperatura en el lecho no isotérmico no adiabático con 58 aproximación de la capa límite y empleando el $15 \%$ de $v_{z}$ en $v_{r}, \operatorname{Re}_{p}=163.36$, a 7 posiciones axiales diferentes; ( $\mathbf{\Delta})$ dato experimental, (-) modelo.

Figura 4.21. Perfil axial de temperatura en el lecho no isotérmico no adiabático con aproximación de la capa límite y empleando el $20 \%$ de $\mathrm{v}_{\mathrm{Z}}$ en $\mathrm{v}_{\mathrm{r}}$, $\mathrm{Re}_{\mathrm{p}}=163.36$, a 3 posiciones radiales diferentes; $(\boldsymbol{\Delta})$ dato experimental, (一) modelo.

Figura 4.22. Perfil radial de temperatura en el lecho no isotérmico no adiabático con aproximación de la capa límite y empleando el $20 \%$ de $\mathrm{v}_{\mathrm{z}}$ en $\mathrm{v}_{\mathrm{r}}$, $\mathrm{Re}_{\mathrm{p}}=163.36$, a 7 posiciones axiales diferentes; $(\boldsymbol{\Delta})$ dato experimental, (-) modelo.

Figura 4.23. Perfil axial de temperatura en el lecho no isotérmico no adiabático con aproximación de la capa límite, empleando el $10 \%$ de $\mathrm{v}_{\mathrm{Z}}$ en $\mathrm{v}_{\mathrm{r}}$, estimación con datos del centro del lecho, $\mathrm{Re}_{\mathrm{p}}=163.36$, a 3 posiciones radiales diferentes.

Figura 4.24. Perfil radial de temperatura en el lecho no isotérmico no adiabático con aproximación de la capa límite, empleando el $10 \%$ de $\mathrm{v}_{\mathrm{z}}$ en $\mathrm{v}_{\mathrm{r}}$, estimación con datos del centro del lecho, $\operatorname{Re}_{\mathrm{p}}=163.36$, a 7 posiciones axiales diferentes.

Figura 4.25. Comparación de los perfiles radiales de temperatura en el lecho no isotérmico no adiabático obtenidos con las diferentes aproximaciones, $\mathrm{Re}_{\mathrm{p}}=163.36, \mathrm{z}=2.5 \mathrm{~cm}$ y $5.61 \mathrm{~cm}$.

Figura 4.26. Comparación de los perfiles radiales de temperatura en el lecho no isotérmico no adiabático obtenidos con las diferentes aproximaciones, $\mathrm{Re}_{\mathrm{p}}=163.36, \mathrm{z}=20.70 \mathrm{~cm}$ y $23.42 \mathrm{~cm}$. 
Figura 4.27. Comparación de los perfiles axiales de temperatura en el lecho no isotérmico no adiabático obtenidos con las diferentes aproximaciones, $\mathrm{Re}_{\mathrm{p}}=163.36, \mathrm{r}=1.27 \mathrm{~cm}$.

Figura 4.28. Comparación de los perfiles axiales de temperatura en el lecho no isotérmico no adiabático obtenidos con las diferentes aproximaciones, $\operatorname{Re}_{\mathrm{p}}=163.36, \mathrm{r}=0 \mathrm{~cm}$.

Figura 4.29. Comparación de los perfiles axiales de temperatura en el lecho no isotérmico no adiabático obtenidos con las diferentes aproximaciones, $\mathrm{Re}_{\mathrm{p}}=163.36$, en $\mathrm{r}=0.3106 \mathrm{~cm}$.

Figura 4.30. Perfil axial de temperatura en el lecho no isotérmico no adiabático con aproximación de la capa límite y empleando el $10 \%$ de $\mathrm{v}_{\mathrm{z}}$ en $\mathrm{v}_{\mathrm{r}}$, $\mathrm{Re}_{\mathrm{p}}=40.83$, a 3 posiciones radiales diferentes; $(\boldsymbol{\Delta})$ dato experimental, (一) modelo.

Figura 4.31. Perfil radial de temperatura en el lecho no isotérmico no adiabático con 68 aproximación de la capa límite y empleando el $10 \%$ de $\mathrm{v}_{\mathrm{z}}$ en $\mathrm{v}_{\mathrm{r}}$, $\mathrm{Re}_{\mathrm{p}}=40.83$, a 7 posiciones axiales diferentes; $(\boldsymbol{\Delta})$ dato experimental, (一) modelo.

Figura 4.32. Perfil axial de temperatura en el lecho no isotérmico no adiabático con 69 aproximación de la capa límite y empleando el $10 \%$ de $\mathrm{v}_{\mathrm{z}}$ en $\mathrm{v}_{\mathrm{r}}$, $\mathrm{Re}_{\mathrm{p}}=81.67$, a 3 posiciones radiales diferentes; $(\mathbf{\Delta})$ dato experimental, (一) modelo.

Figura 4.33. Perfil radial de temperatura en el lecho no isotérmico no adiabático con aproximación de la capa límite y empleando el $10 \%$ de $\mathrm{v}_{\mathrm{z}}$ en $\mathrm{v}_{\mathrm{r}}$, $\mathrm{Re}_{\mathrm{p}}=40.83$, a 7 posiciones axiales diferentes; $(\boldsymbol{\Delta})$ dato experimental, (一) modelo. 
Índice de Tablas

Tabla 3.1. Condiciones de experimentación del caso 1.

Tabla 3.2. Condiciones de experimentación del caso 2.

Tabla 4.1 keff,z en el lecho quasi-adiabático, modelo pseudo-homogéneo.

Tabla 4.2 Parámetros térmicos efectivos en el lecho quasi-adiabático, modelo pseudo-heterogéneo.

Tabla 4.3. $\quad k_{\text {eff,z }}$ de experimento dinámico, modelo pseudo-homogéneo.

Tabla 4.4. Parámetros térmicos efectivos del experimento dinámico, modelo pseudo-heterogéneo. 


\section{Capítulo I - Introducción}

El craqueo térmico es un proceso industrial que ha predominado en la producción de etileno, una de las olefinas más utilizadas como intermediarias para la formación de diversos productos, tales como: plásticos, resinas, fibras, polímeros, entre otros compuestos importantes. Sin embargo, este proceso, al ser altamente endotérmico, requiere de altas temperaturas $\left(800^{\circ} \mathrm{C}\right)$ para llevar a cabo la descomposición térmica de hidrocarburos. No obstante, en esta tecnología se tiene también la producción no deseada de cantidades significativas de $\mathrm{CO}_{2}$ y $\mathrm{NO}_{\mathrm{x}}$, y un costo energético aproximado del $70 \%$ del costo total de la producción.

Dado el impacto ambiental y energético (económico) del craqueo térmico, ha surgido la necesidad de implementar nuevos procesos para la producción de etileno, en donde la deshidrogenación oxidativa de etano (DHO) es una opción prometedora, no sólo por ser un proceso exotérmico, el cual se podría llevar a cabo a temperaturas menores a $\operatorname{los} 500^{\circ} \mathrm{C}$, con los catalizadores adecuados, sino también porque termodinámicamente no está limitada ${ }^{1}$.

La DHO y otro tipo de oxidaciones parciales de hidrocarburos son procesos altamente exotérmicos, por lo que se ha propuesto que se lleven a cabo en reactores de lecho empacado con relaciones de diámetro de tubo diámetro de partícula menores a $10\left(\mathrm{~d}_{\mathrm{t}} / \mathrm{d}_{\mathrm{p}}<10\right)$. No obstante, esta relación geométrica hace que se generen perfiles de fracción vacía y velocidad que interactúan de manera compleja con los distintos mecanismos de transferencia de calor, masa y reacción ${ }^{1-3}$. Por otro lado, este tipo de configuración geométrica permite que el calor generado por las reacciones se disipe hacia el fluido enfriante, ya que la generación de puntos calientes podría afectar el rendimiento deseado de la reacción o inclusive desactivar el catalizador por un mecanismo de sinterizado.

Actualmente, la academia no ha podido desarrollar un modelo generalizado que describa el comportamiento de esta clase de reactores, señalando que las principales limitantes para el desarrollo de este modelo son la descripción inadecuada de la hidrodinámica y su efecto en la transferencia de calor.

Particularmente, a pesar de que exista en la literatura una cantidad significativa de estudios

de transferencia de calor en lechos empacados en ausencia de reacción, aún se tienen 
bastantes limitantes para describir los mecanismos correspondientes. Las principales limitaciones que se han encontrado en los distintos trabajos de transferencia de calor para la estimación de los parámetros efectivos, $\mathrm{k}_{\mathrm{eff}} \mathrm{y} \mathrm{h}_{\mathrm{w}}$, en este tipo de sistemas son: se tiene una alta correlación estadística entre los parámetros de transferencia de calor estimados, la hidrodinámica es despreciada, esencialmente la contribución de la velocidad radial, y no se tiene claro su papel en la transferencia de calor, no se ha evaluado apropiadamente la contribución estática y dinámica de los distintos mecanismos de transferencia de calor, se tienen mediciones experimentales insuficientes del campo de temperatura, se usan modelos pseudo-homogéneos que aparentemente no capturan los mecanismos de transferencia de calor del sólido, se utilizan observaciones donde los gradientes de temperaturas son menores a aquellos que se tienen cuando hay reacción, la estimación de parámetros se lleva a cabo en estado estacionario, se tiene incertidumbre en las diversas correlaciones reportadas en la literatura, y no se ha descrito adecuadamente la transitoriedad de la transferencia de calor.

Mencionado lo anterior, el presente estudio esclarece algunas de las limitantes históricas al estimar los parámetros efectivos de transferencia de calor en un lecho empacado quasiadiabático y un lecho empacado con calentamiento en la pared. Para lograr esto, se plantea una familia de modelos que permitan caracterizar la transferencia de calor en estado transitorio y estacionario para cada lecho, y se compara con datos experimentales obtenidos de trabajos anteriores del grupo de investigación ${ }^{4,5}$.

En el capítulo 2 del presente trabajo se describen brevemente los mecanismos de transferencia de calor que suceden en un lecho empacado, así también se hace mención a las limitantes históricas que se han tenido durante la determinación de los parámetros efectivos de transferencia de calor y se da una descripción general de los estudios teóricos y experimentales que se realizan en este tipo de sistemas.

El capítulo 3 está dividido en 3 secciones, en la primera sección se describen los sistemas experimentales que se estudiaron en este trabajo, en la segunda sección se describen las condiciones de operación empleadas en los sistemas experimentales, y en la última sección se describen los modelos empleados para predecir los perfiles de temperatura observados en los sistemas experimentales. 
Los resultados obtenidos en este trabajo se presentan en el capítulo 4. Primero se presenta la caracterización de la transferencia de calor en el lecho quasi-adiabático y después para el lecho no isotérmico y no adiabático. Por último, en este capítulo, se realiza una comparación y análisis sobre las aproximaciones empleadas para describir la transferencia de calor en el lecho con calentamiento en la pared.

En el capítulo 5 se presentan las conclusiones obtenidas del trabajo realizado en este proyecto. Finalmente, en el capítulo 6 se presentan apéndices de este trabajo. 


\section{Objetivos}

\section{Objetivo General}

Caracterizar la transferencia de calor en ausencia de reacción en un lecho empacado con baja relación de $d_{t} / d_{p}$ que se opera de manera quasi-adiabática y con calentamiento en la pared.

\section{Objetivos Particulares}

- Describir la transferencia de calor en estado estacionario y transitorio en el lecho empacado, quasi-adiabático y con calentamiento en la pared, considerando la pseudoheterogeneidad y la pseudohomogeneidad.

- Describir la transferencia de calor que se observa en experimentos dinámicos del lecho quasi-adiabático.

- Estimar los parámetros de transferencia de calor en el lecho, para el caso donde existan perfiles de temperatura similares a los que se tienen con reacción y se considere la las componentes axial y radial de la velocidad.

- Evaluar la contribución estática y dinámica para los modelos del lecho quasiadiabático y con calentamiento en la pared. 
Capítulo II - Transferencia de calor en lechos empacados

\section{$\underline{2.1 \text { Generalidades }}$}

La caracterización de la transferencia de calor, acoplada o no a otro tipo de mecanismos de transporte o reacción, es fundamental para la descripción de múltiples procesos unitarios en la industria, tales como: reactores químicos, secadores, torres de adsorción, entre otros. Es tal su importancia, que para caracterizar estos mecanismos de transferencia de calor, se realizan estudios independientes, en donde se aísla el transporte de calor de otros mecanismos que puedan perturbar su caracterización intrínseca. En el caso de los lechos empacados, se puede dividir en tres tipos de estudios de transferencia de calor, clasificándolos por la forma en la que se añade o remueve calor del sistema; en la entrada o salida, en las paredes, y dentro del lecho ${ }^{6}$. Cabe señalar que esta es una clasificación muy general, debido a que en cada estudio se debe de especificar la siguiente información:

- Si son experimentales y/o teóricos.

- Si consideran el equilibrio térmico entre ambas fases o no.

- Si la lectura realizada de los datos experimentales, temperatura, fue tomada específicamente para cada fase.

- Si fue realizado de manera transitoria o en estado estacionario.

- La relación de diámetro de tubo diámetro de partícula del sistema $\left(\mathrm{d}_{\mathrm{t}} / \mathrm{d}_{\mathrm{p}}\right)$.

- El fluido empleado.

- El material usado como empaque, así como su forma.

- El flujo manejado $\left(\operatorname{Re}_{\mathrm{p}}\right)$.

- Los gradientes de temperatura encontrados en el lecho.

- La consideración de la dispersión axial y/o radial, así como también el coeficiente de transferencia de calor interfacial y/o de pared.

- El tipo de campo de velocidad que se tomó en cuenta.

Por las especificaciones señaladas anteriormente y debido a que en la literatura se encuentran una gran cantidad de correlaciones para diferentes sistemas en donde, a veces, no se llega a encontrar información suficiente del sistema experimental empleado. Es de gran importancia 
señalar que cada sistema experimental debe de tener sus propias correlaciones para calcular con confianza los parámetros que caracterizan la transferencia de calor.

Para la descripción de la transferencia de calor en un sistema se utilizan modelos matemáticos, donde, por las ventajas de recursos computacionales, se emplean los modelos pseudo-continuos. Al ser modelos "pseudo", se emplean parámetros "efectivos" que describen fenomenológicamente los mecanismos de transferencia de calor y/o masa que sucedan dentro del sistema. Estos modelos pueden ser unidimensionales (dirección axial) o en dos dimensiones (dirección axial y radial).

Uno de los parámetros que definen la transferencia de calor en un lecho empacado se conoce como la conductividad térmica efectiva ( $\mathrm{k}_{\text {eff }}$ ), en donde el transporte de calor en la dirección axial o radial es posible, por lo que este parámetro se puede especificar como conductividad térmica efectiva axial $\left(\mathrm{k}_{\mathrm{eff}, \mathrm{z}}\right)$ y radial $\left(\mathrm{k}_{\mathrm{eff}, \mathrm{r}}\right)$. Es importante resaltar que existen casos en donde la transferencia de calor en una dirección predomina. El transporte de calor radial en un lecho empacado también tiene lugar en la pared del tubo, por lo que es necesario determinar un coeficiente de transporte de calor de pared $\left(\mathrm{h}_{\mathrm{w}}\right)$ en esa zona para la descripción adecuada del sistema, este coeficiente representa la conducción por contacto de una partícula y la pared, la radiación entre la partícula y la pared, y el transporte a través de una película libre ${ }^{7}$. Estos mecanismos tienen lugar también entre la pared del tubo y el fluido enfriante.

La conductividad térmica efectiva se ejemplifica por la dispersión térmica en el empaquetamiento de partículas sólidas discretas en un fluido que puede ser estático o en estado de flujo. Puede definirse como la densidad total de flujo de calor dividida por la diferencia de temperatura global en la dirección del flujo y, por lo tanto, es una propiedad que depende del estado físico del sistema.

En este sentido, la conductividad térmica efectiva engloba varios mecanismos de transporte de calor dependientes e independientes del flujo del fluido, ver Figura 2.1, descritos a continuación ${ }^{7,8}$ :

Mecanismos independientes del flujo del fluido:

1. Conducción a través del fluido

2. Conducción por contacto entre partículas 
3. Radiación entre los huecos del lecho (fracción vacía)

4. Radicación entre las superficies de los sólidos.

5. Conducción a través del sólido.

Mecanismos dependientes del flujo del fluido:

6. Convección por el fluido en dirección del flujo del fluido.

7. Conducción a través de la película de fluido cerca de la superficie de contacto de dos partículas.

8. Transferencia de calor por el mezclado transversal y longitudinal del fluido.

9. Transferencia de calor por convección sólido-fluido-sólido.

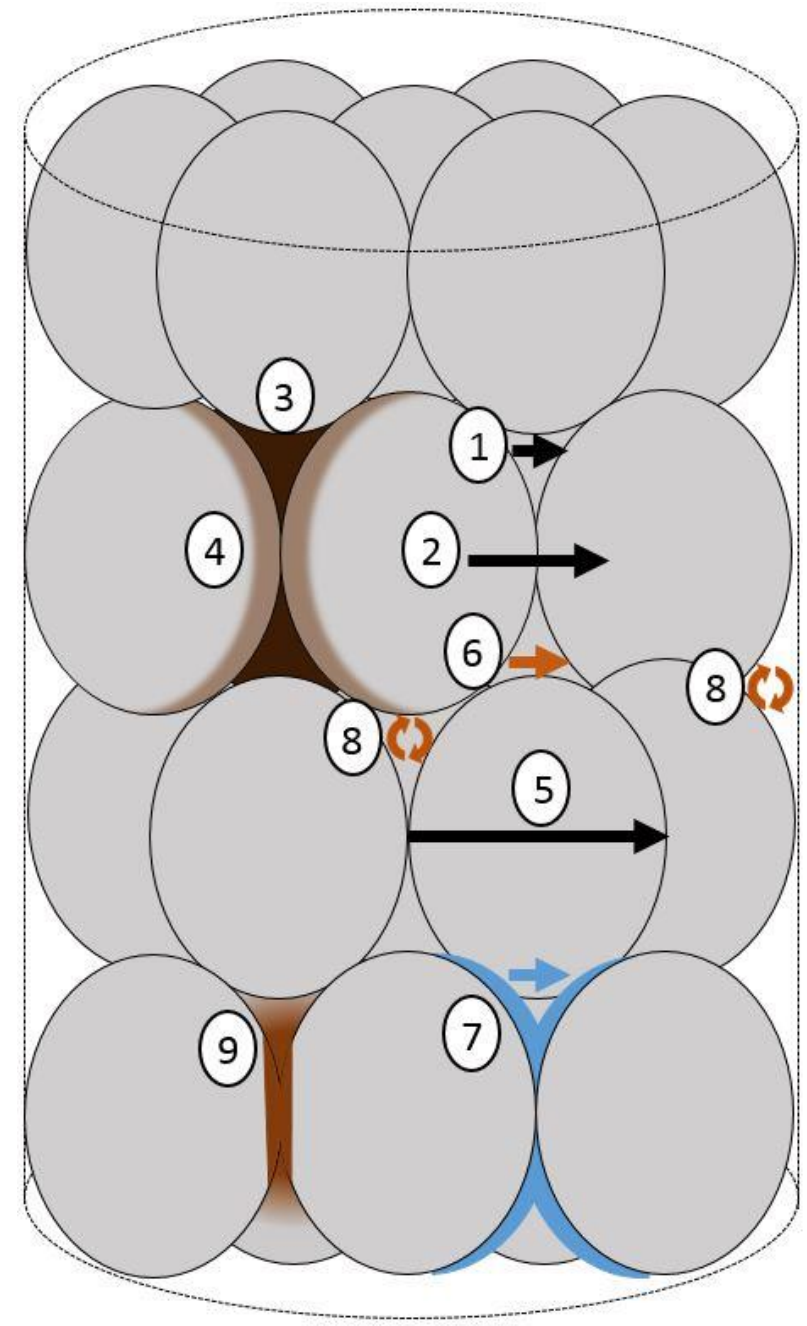

Figura 2.1. Mecanismos de transporte de calor que engloba la conductividad térmica efectiva, $\mathrm{k}_{\text {eff. }}$ 


\section{$\underline{\text { 2.2 Transporte de calor axial }}$}

Normalmente, el efecto de la conductividad térmica axial se ignora en los cálculos del diseño de reactores de lecho empacado, debido a que la transferencia de calor radial hacia las paredes del reactor (dirección radial) domina sobre la transferencia en dirección axial. Sin embargo, es deseable desarrollar un procedimiento que indique la importancia relativa de la conductividad axial y que pueda utilizarse para justificar su descuido, es por eso que existen diversos trabajos en la literatura en donde se realizan experimentos con paredes quasiadiabáticas para únicamente obtener la información de la transferencia de calor axial.,9-11 Sin embargo, como se mencionó anteriormente, existe una diversidad de correlaciones para distintos lechos empacados ${ }^{9,11-16}$ como se ilustra en la Figura 2.2, por lo que puede no ser correcto el emplear alguna correlación propuesta en la literatura para el sistema a estudiar, esto incluso es más evidente cuando se trabaja con lechos empacados que presentan una relación de $\mathrm{dt} / \mathrm{dp}$ menor a $10^{2,3,5,14,17,18}$.

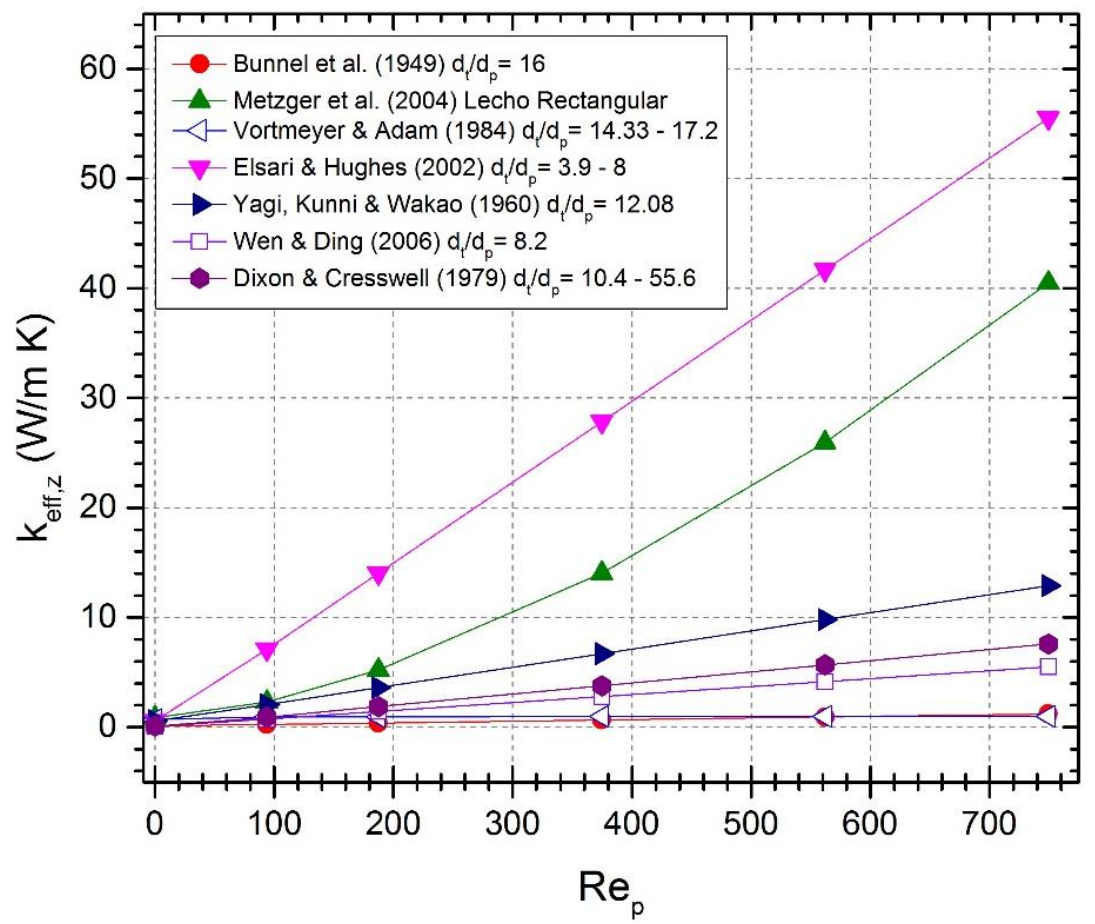

Figura 2.2. Correlaciones para obtener el parámetro $k_{\mathrm{eff}, \mathrm{z}}$ a diferentes $\operatorname{Re}_{\mathrm{p}}$.

\section{$\underline{\text { 2.3 Transporte de calor radial }}$}

Para describir el transporte de transferencia de calor en dirección radial históricamente se han empleado dos tipos de modelos ${ }^{7}$ : 
- Modelo unidimensional.- En este tipo de modelos se calcula un coeficiente global de transferencia de calor $(\mathrm{U})$, sin embargo, únicamente con esta aproximación se tiene resultados adecuados cuando no se presentan gradientes apreciables de temperatura, perfil radial plano, en la región central del lecho, esto debido a que toda la resistencia a la transferencia de calor se localiza en la región cercana a la pared, por lo que no se requiere de una $\mathrm{k}_{\mathrm{eff}, \mathrm{r}}$.

- Modelo bidimensional con $\mathrm{h}_{\mathrm{w}} \mathrm{y}$ keff,r-- Este tipo de modelos se utiliza cuando hay gradientes del temperatura apreciables entre el centro del lecho y la pared, más aún, cuando existe un gradiente de temperatura en la región cercana a la pared mayor que el gradiente de temperatura que se tiene en la zona central del lecho.

Cabe señalar que estas aproximaciones consideran parámetros efectivos. De manera general pareciera que el modelo bidimensional con $\mathrm{h}_{\mathrm{w}} \mathrm{y} \mathrm{k}_{\mathrm{eff}, \mathrm{r}}$ fuese la mejor opción, sin embargo, investigadores en la literatura ${ }^{19-23}$ afirman que la keff,r debería de estar en función de la posición radial en el lecho y que se debe de prescindir del $\mathrm{h}_{\mathrm{w}}$ en la condición de frontera de la pared y utilizar la condición de frontera donde la temperatura es la de la pared, $\mathrm{T}=\mathrm{T}_{\mathrm{w}}$, lo cual también sugiere una incorporación de la variación de fracción vacía y de la velocidad en su componente axial. No obstante, debido a que la hidrodinámica no se describe adecuadamente en esta zona del lecho, se puede llegar a utilizar la aproximación de la capa límite, la cual vuelve a incorporar un $\mathrm{h}_{\mathrm{w}}$ en la condición de frontera, de tal forma que ya no se necesita conocer la temperatura de la pared pero si la del baño de enfriamiento, sin embargo, este parámetro representa el transporte de calor a través de las capas límites térmicas en ambos lados de la pared del lecho., ${ }^{2,5}$

También cabe señalar que en la literatura existen una gran diversidad de correlaciones para estos parámetros efectivos, $\mathrm{k}_{\mathrm{eff}, \mathrm{r}} \mathrm{y} \mathrm{h}_{\mathrm{w}}$, como se muestran en las figuras 2.3 y 2.4. Debido a la incertidumbre que se tiene con estos parámetros que son característicos de cada prototipo experimental, y, como se mencionó anteriormente con la keff,z, también se deben de calcular dichos parámetros para el sistema de interés. 


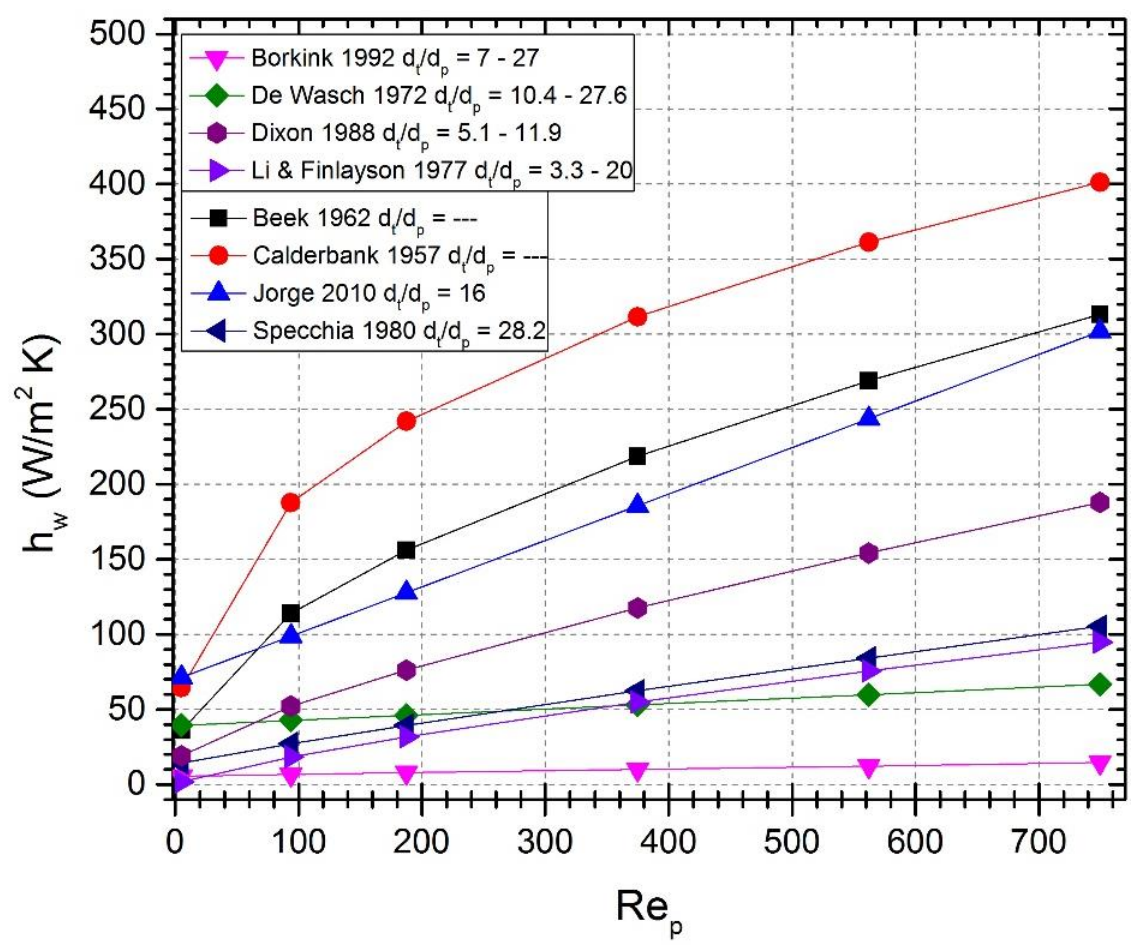

Figura 2.3. Correlaciones para obtener el parámetro $h_{w}$ a diferentes $\operatorname{Re}_{\mathrm{p}}$.

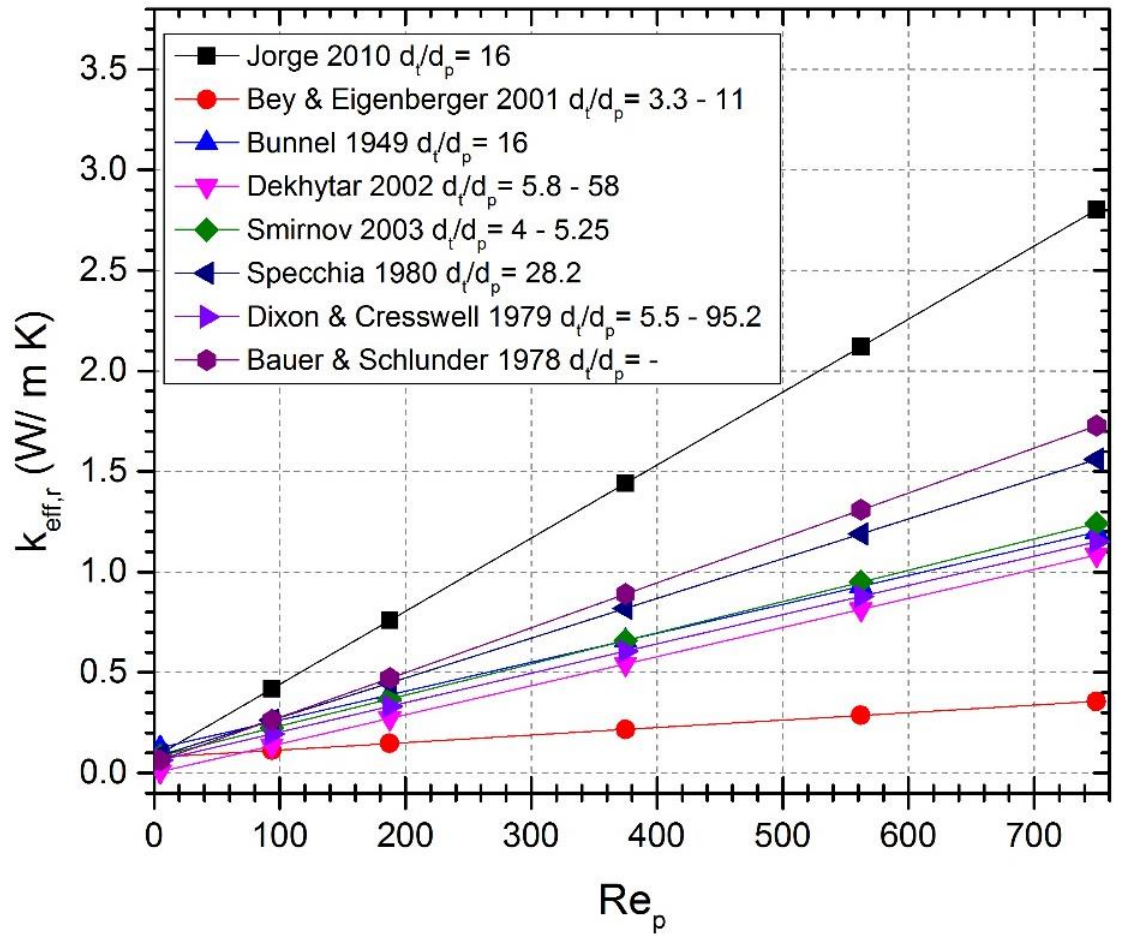

Figura 2.4. Correlaciones para obtener el parámetro $k_{\mathrm{eff}, \mathrm{r}}$ a diferentes $\mathrm{Re}_{\mathrm{p}}$. 


\subsection{Limitantes históricas para la caracterización de la transferencia de calor en lechos}

\section{empacados}

Las principales limitantes históricas que se han encontrado en los distintos trabajos de transferencia de calor para la caracterización de la transferencia de calor, y por lo tanto la estimación de parámetros efectivos, $\mathrm{k}_{\text {eff }} \mathrm{y} \mathrm{h}_{\mathrm{w}}$, en este tipo de sistemas, se describen a continuación:

-Alta correlación estadística: Como se muestra en la Figura 2.5, se pueden estimar bastantes combinaciones de parámetros efectivos, $\mathrm{h}_{\mathrm{w}} \mathrm{y} \mathrm{k}_{\mathrm{eff}}$, que ajusten observaciones de la transferencia de calor, pero que fenomenológicamente describan algo que no esté pasando en el sistema, esto sucede debido a que los parámetros efectivos son obtenidos del mismo conjunto de datos experimentales, es decir, existe una alta correlación estadística entre ambos parámetros $^{5,24}$. Lo que se ha hecho en los trabajos de la literatura, es encontrar una región de confianza en donde se contenga la mayor probabilidad de que estos parámetros ajusten mejor a los valores experimentales, región más oscura de la figura 2.5 , esto se ha realizado mediante diversos métodos que minimicen una función objetivo ${ }^{15,24-28}$.

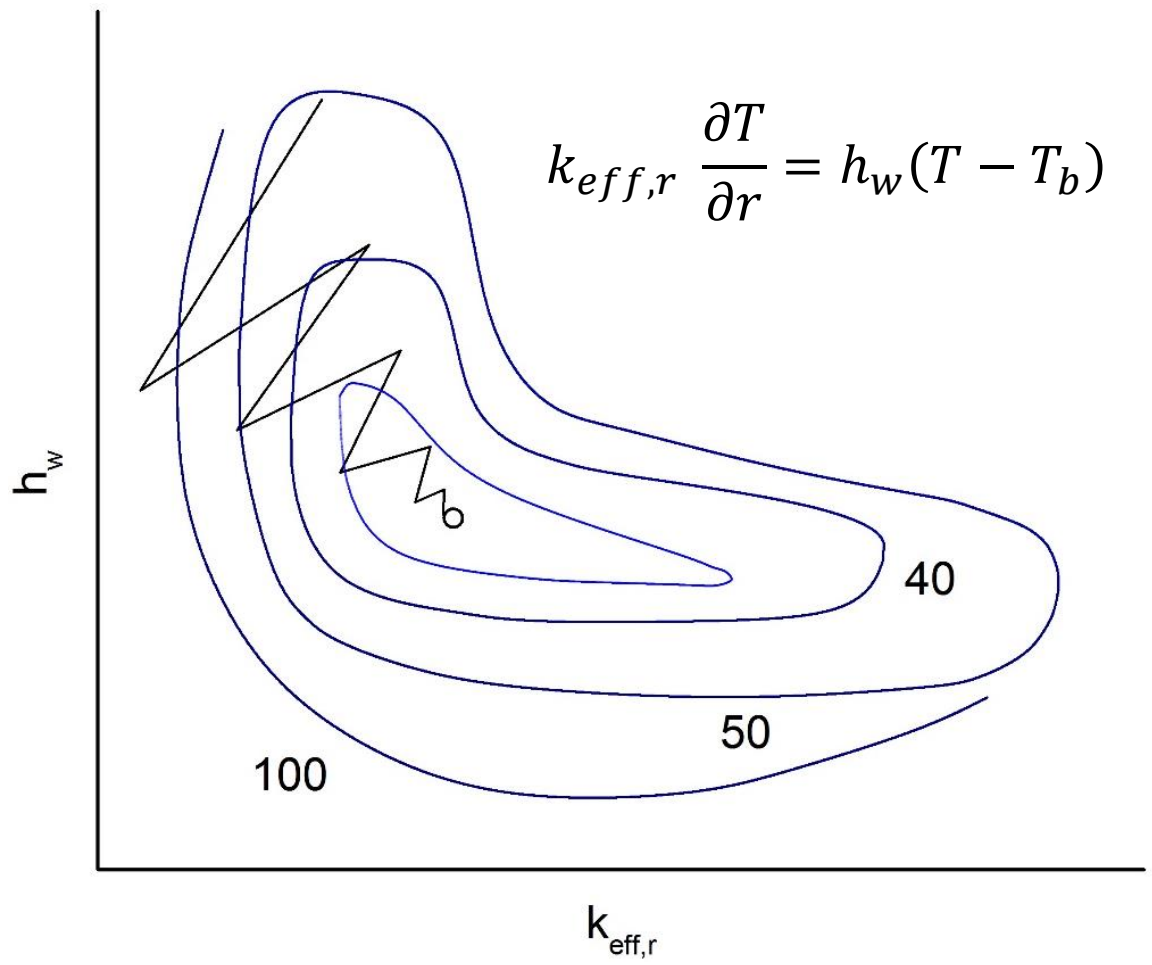

Figura 2.5. Superficie de error tridimensional $\left(\mathrm{F}, \mathrm{h}_{\mathrm{w}}\right.$ y $\left.\mathrm{k}_{\mathrm{eff}, \mathrm{r}}\right)$ en un lecho empacado. 
-Hidrodinámica despreciada: La mayoría de modelos encontrados en los trabajos de literatura, con y sin reacción, desprecian la cuestión hidrodinámica dentro del lecho empacado $^{8,9,15,16,29-37}$, pues se considera un flujo pistón, y cuando se considera la hidrodinámica se desprecia la componente radial de la velocidad justificando un gradiente de presión radial despreciable $e^{1,2,5,19,23,38-40}$, el cual aparenta tener un efecto importante en $^{41,42}$.

En la Figura 2.6 se muestran predicciones reportadas en trabajos experimentales ${ }^{2}$ en donde se compara un modelo de transferencia de calor que considera flujo pistón y uno que considera hidrodinámica en su componente axial. La velocidad dentro de un lecho empacado con baja relación $d_{t} / d_{p}$ puede ser inclusive 10 veces mayor o casi cero dependiendo de su posición radial, como se muestra en la Figura 2.7, por lo que el perfil de velocidad juega un papel importante en los mecanismos de transferencia de calor.

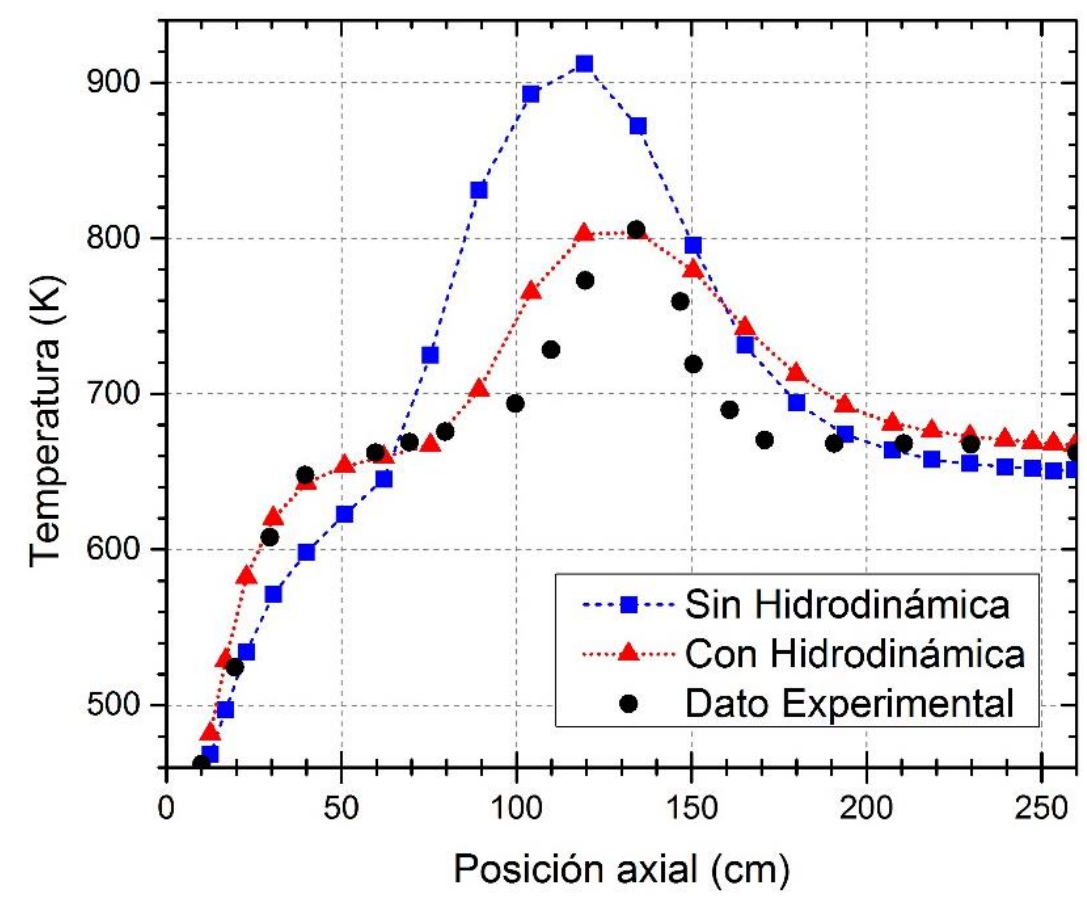

Figura 2.6. Perfiles de temperatura en un experimento con reacción de oxidación de oXileno a $\mathrm{Re}_{\mathrm{p}}=630, \mathrm{~T}_{\text {entrada }}=463 \mathrm{~K}, \mathrm{~T}_{\text {baño }}=665 \mathrm{~K}$ : flujo pistón $\mathbf{m}$, hidrodinámica (componente axial), datos experimentales $\bullet$. 


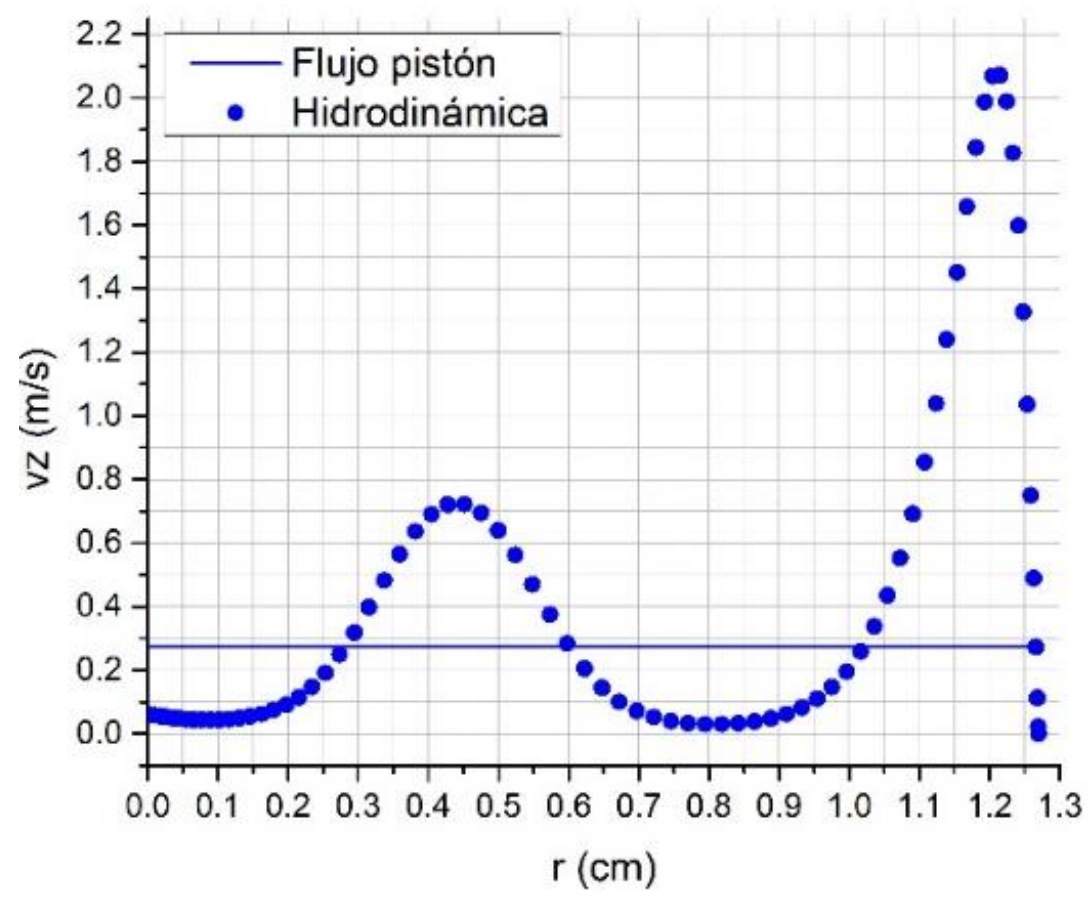

Figura 2.7. Perfiles de velocidad axial de aire dentro de un lecho empacado con relación $\mathrm{d}_{\mathrm{t}} / \mathrm{d}_{\mathrm{p}}=3.175, \mathrm{Re}_{\mathrm{p}}=163.36$, considerando la hidrodinámica $($ componente axial) $(\bullet)$, considerando flujo pistón (一).

-Parámetros medios efectivos: La mayoría de los modelos de transferencia de calor con calentamiento en la pared ha considerado la conductividad térmica efectiva radial como una media valida en todo el sistema ${ }^{12,16,24,39,43-45}$, en donde los perfiles de fracción vacía y de velocidad dentro del lecho, ver figura 2.7, juegan un papel importante en los mecanismos de transferencia de calor hacia la pared, por lo que varios investigadores ${ }^{20,21,31}$ creen que este parámetro debería estar en función de la posición radial, así como del $\mathrm{Re}_{\mathrm{p}}$, lo cual, permitirá obtener información valiosa para describir la transferencia de calor en esa región del lecho.

-Evaluación de la contribución estática y dinámica: Son pocos los trabajos que evalúan la contribución estática y dinámica de los parámetros efectivos de transferencia de calor en un lecho empacado ${ }^{20,21,31}$, por lo que se cree que evaluando ambas contribuciones en función de la posición radial y del $\operatorname{Re}_{\mathrm{p}}$ se podrá identificar y capturar la información dentro del sistema, lo que permitiría describir la transferencia de calor.

-Mediciones experimentales insuficientes del campo de temperatura: A pesar de que existan varias técnicas para tomar datos experimentales de temperatura dentro de un lecho 
empacado con calentamiento en la pared, la mayoría de los trabajos experimentales de transferencia de calor encontrados en la literatura únicamente toman mediciones experimentales de temperatura en el centro y/o pared del lecho a lo largo del mismo ${ }^{14,19,31,46}$, o sólo en la entrada y/o salida del lecho a diferentes posiciones radiales ${ }^{17,28,31,36,47-50}$, esto ha llevado a una falta de información de transferencia de calor dentro del lecho que repercute en una deficiente representación cuantitativa de los datos experimentales y a su vez, una mala estimación de los parámetros efectivos de transferencia de calor.

-Modelos pseudo-homogéneos: La mayoría de los estudios en la actualidad se han llevado a cabo usando modelos pseudo-homogéneos ${ }^{5,8,9,23,28,38,40,50,51}$, los cuales no hacen distinción entre la temperatura de la fase fluida y la fase sólida. Esta diferencia de temperatura entre ambas fases, exista o no, generalmente se desconoce a nivel experimental, por lo que la suposición de un equilibrio térmico local es puesta en duda y más para estudios transitorios, donde la contribución del sólido tiene mayor efecto. ${ }^{15}$

-Gradientes de temperatura: Normalmente los parámetros efectivos son estimados bajo gradientes de temperatura mucho menores que los existentes cuando hay reacción ${ }^{5}$, como se puede ver en la Figura $2.8^{2,5}$, en el caso a) se tienen gradientes de casi $400^{\circ} \mathrm{C}$ para un sistema con reacción, mientras que para el caso b) se tiene el mismo sistema pero sin reacción y con paredes quasi-adiabáticas, en donde el gradiente de temperatura no es mayor a $\operatorname{los} 50^{\circ} \mathrm{C}$ a Rep de 630, como en el caso de reacción. Hasta la fecha no se tiene claro cuál es el efecto de los gradientes de temperatura en la estimación de los parámetros. 

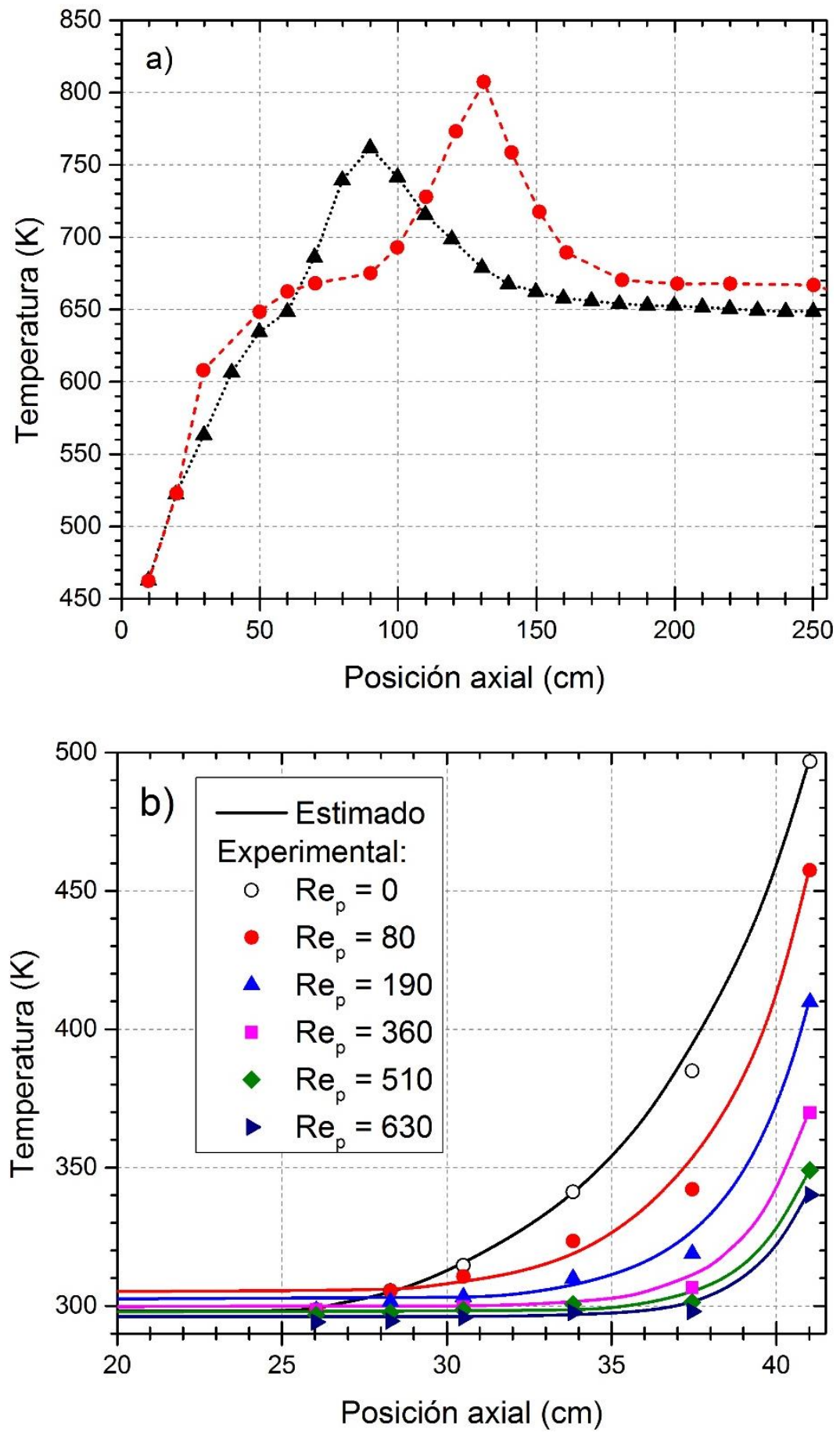

Figura 2.8. a) Perfil de temperatura de 2 experimentos de oxidación de o-Xileno a $\left.\mathrm{Re}_{\mathrm{p}}=630, \mathrm{~T}_{0}=463 \mathrm{~K}, \mathrm{~T}_{\mathrm{b}}=665 \mathrm{~K}(\bullet), \mathrm{T}_{\mathrm{b}}=649 \mathrm{~K}(\boldsymbol{\Delta}), \mathrm{b}\right)$ Perfiles de temperatura en un lecho adiabático. 
-Estimación en estado estacionario: La estimación de parámetros efectivos de transferencia de calor se ha llevado a cabo esencialmente en estado estacionario ${ }^{5,9-12,14,24,52}$. Debido a que los gradientes de temperatura van cambiando a través del tiempo hasta llegar al estado estacionario, se considera que los parámetros pueden ser diferentes si se estiman en estado transitorio.

-Diversas correlaciones en la literatura: En la literatura se pueden encontrar bastantes correlaciones para obtener los diversos parámetros de transferencia de calor, sin embargo, muchas veces no se especifica el sistema ni las propiedades de los materiales con los cuales fueron obtenidos, habiendo bastante diferencia entre los valores dados por las diferentes correlaciones $^{40}$, como se mostró en las figuras 2.2-2.4.

-Descripción de la transitoriedad: La gran mayoría de los trabajos que estudian la transferencia de calor en estado transitorio lo hacen únicamente de manera experimental ${ }^{15,53,54}$. Son muy escasos los trabajos que modelan la transitoriedad en la transferencia de calor de un lecho empacado y que comparan con datos experimentales. Sin embargo, esta ha sido descrita considerando convección libre ${ }^{55-57}$, en donde el flujo de un fluido es prácticamente despreciable, y se ha llegado a considerar un mecanismo de transporte convectivo, se ha estimado un parámetro que engloba datos del sistema cuyas propiedades físicas y termofísicas pueden ser obtenidas sin dificultad de manera independiente, por lo que es puesto en duda la validez del uso de dicho parámetro.

\section{$\underline{\text { 2.5 Estudios realizados en lechos empacados }}$}

A continuación se mencionaran algunos estudios de transferencia de calor realizados en lechos empacados en la literatura a lo largo del siglo XX y siglo XXI, la intención es mostrar la forma en la que se ha estado abordando el problema en estos sistemas, y su evolución.

Yagi y $\mathrm{Kuni}^{8}$ en 1957 y Yagi et al. en 1960 , fueron de los primeros autores que investigaron la conductividad térmica efectiva en un lecho empacado. Utilizaron dos sistemas experimentales, el primer lecho empacado constaba de dos tubos concéntricos, el tubo exterior era de acero inoxidable con un diámetro interno de $14.6 \mathrm{~cm}$, mientras que el tubo interior era de sílice con un diámetro interno de $3.75 \mathrm{~cm}$, que a su vez contenía un calentador eléctrico de carborundo, los empaques se encontraban entre ambos tubos y la transferencia 
de calor tenía lugar desde el centro del lecho hacía la pared externa del tubo de acero, el cual estaba recubierto por ladrillos aisladores de fuego, por lo que se evitaron las pérdidas de calor a lo largo del eje, de tal forma que la distribución de temperatura fue constante; los termopares fueron colocados cerca de la pared de acero. El segundo lecho fue un tubo de 58 $\mathrm{mm}$ de diámetro interno con doble pared de vidrio, donde la superficie interna fue cubierta con plata para aislar la transferencia de calor radial hacia la pared, la fuente de calor fue proporcionada por una lámpara infrarroja en la parte superior del lecho, los termopares fueron colocados en el centro del lecho a distintas posiciones axiales y el flujo de aire fue alimentado por la parte inferior del lecho. Cabe señalar que esta idea del lecho adiabático es uno de los más empleados en la literatura para capturar la información que describa la transferencia de calor en dirección axial.

En estos sistemas empleados, se discute sobre los mecanismos de transferencia de calor dependientes e independientes del flujo del fluido, mencionados anteriormente en la sección 2.1 , señalando que a flujos bajos $\left(\operatorname{Re}_{\mathrm{p}}<5\right)$ predominan los mecanismos de conducción a través de un sólido, radiación entre dos superficies de sólidos, radiación entre la fracción vacía y la conducción térmica a través de la película del fluido cerca del área de contacto entre dos partículas, siendo este último mecanismo libre de ser afectado a flujos altos también, mientras que a flujos altos predomina la transferencia de calor por el retromezclado del fluido. También consideraron que el mecanismo de transferencia de calor de sólido-fluido-sólido puede ser despreciado con seguridad al no ser importante, lo cual justificaba a los modelos pseudo-homogéneos.

Hasta este punto, se podría cuestionar sobre su suposición de despreciar la transferencia de calor de sólido-fluido-sólido debido a que no se contaba con mediciones experimentales adecuadas para cada fase, también es cuestionable el uso de la radiación térmica debido al empleo de temperaturas relativamente bajas en los experimentos.

A pesar de las limitantes que se pudieran haber tenido para realizar dichos trabajos, sin duda alguna, una de sus contribuciones más notorias para la comunidad científica fueron sus correlaciones para la conductividad térmica efectiva axial y radial, con sus respectivos términos dependientes e independientes del flujo, mostrados a continuación. 


$$
\begin{aligned}
& \frac{k_{e f f, z}}{k_{f}}=\frac{k_{e f f, z}^{0}}{k_{f}}+\delta \operatorname{PrR} e_{p} \\
& \frac{k_{e f f, r}}{k_{f}}=\frac{k_{e f f, r}^{0}}{k_{f}}+\alpha \beta \operatorname{Pr} \operatorname{Re}_{p}
\end{aligned}
$$

En donde el superíndice 0 indica un flujo de $\operatorname{Re}_{\mathrm{p}}<5$ (contribución estática), mientras que los subíndices z, r y f indician la posición axial, radial y la fase fluida, respectivamente. A pesar de que estas correlaciones, estrictamente, sólo deberían de funcionar para el sistema que ellos emplearon, con su valor de $\alpha, \beta$ y $\delta$, diversos investigadores han tenido buenos resultados con estas correlaciones, quizás, por emplear un sistema con configuración geométrica y materiales similares.

La mayoría de los modelos en estado estacionario empleados en los trabajos que estudian la transferencia de calor en este tipo de sistemas son similares a los empleados por De Wasch y Froment en su trabajo de $1972^{28}$, en este, describen el perfil de temperaturas dentro de un lecho empacado mediante un modelo unidimensional y un modelo bidimensional, en este último utilizan dos aproximaciones, uno con el parámetro de $\mathrm{k}_{\text {eff,r }} \mathrm{y}$ otro con los parámetros $\mathrm{k}_{\mathrm{eff}, \mathrm{r}} \mathrm{y} \mathrm{h}_{\mathrm{w}}$. El sistema experimental fue muy parecido al utilizado por Yagi y $\mathrm{Kuni}^{8}$, que constaba de dos tubos concéntricos, en este caso, ambos tubos eran de cobre, por el tubo interno circulaba vapor saturado a $100^{\circ} \mathrm{C}$, como fuente de calor, el empaque consistía de partículas de pentóxido de Vanadio $\left(\mathrm{V}_{2} \mathrm{O}_{5}\right)$ con un diámetro de $5.7 \mathrm{~mm}$, el fluido introducido por la parte inferior del lecho era aire, y las temperaturas experimentales únicamente fueron medidas a la salida del lecho.

El modelo utilizado fue uno unidimensional que tenía el siguiente balance de energía:

$$
\frac{R_{t} G C_{p}}{2} \frac{d T}{d z}=U_{w}\left(T_{w}-T\right)
$$

Y sus respectivas condiciones de frontera:

$$
z=0 ; \quad T=T_{\text {in }}
$$

donde $R_{t}$ es el radio interior del tubo exterior, $G$ es el flujo másico superficial, $C_{p}$ es la capacidad calorífica del fluido, $\mathrm{U}$ es el coeficiente de transferencia de calor global, el subíndice $\mathrm{w}$ indica la pared, y $\mathrm{T}_{\mathrm{w}}$ es constante en toda la pared. Como se explicó 
anteriormente, este tipo de modelos tiene resultados favorables cuando el gradiente de temperatura en la zona central del lecho no es apreciable.

El segundo modelo empleado fue un modelo bidimensional con el siguiente balance de energía:

$$
G C_{p} \frac{\partial T}{\partial z}=k_{e}\left(\frac{\partial^{2} T}{\partial r^{2}}+\frac{1}{r} \frac{\partial T}{\partial r}\right)
$$

donde $\mathrm{k}_{\mathrm{e}}$ es la conductividad efectiva global, invariante de la posición axial o radial, ya que se considera al sistema isotrópico. Para este modelo, los autores propusieron dos casos, en el primero, únicamente consideraban que el parámetro ke podía describir los mecanismos de transferencia de calor en el lecho mediante las siguientes condiciones de frontera:

$$
\begin{aligned}
& z=0 ; \quad T=T_{0} \\
& r=0 ; \quad \frac{\partial T}{\partial r}=0 \\
& r=R_{t} ; \quad T=T_{w}
\end{aligned}
$$

Y para el segundo caso, consideraban al parámetro $\mathrm{k}_{\mathrm{e}} \mathrm{y} \mathrm{h}_{\mathrm{w}}$, en donde las condiciones de frontera empleadas son:

$$
\begin{gathered}
z=0 ; \quad T=T_{0} \\
r=0 ; \quad \frac{\partial T}{\partial r}=0 \\
r=R_{t} ; \quad h_{w}\left(T_{w}-T\right)=k_{e} \frac{\partial T}{\partial r}
\end{gathered}
$$

Concentrándose en el segundo modelo, que es el de mayor interés, en este estudio los autores resuelven el modelo propuesto con sus respectivas condiciones de frontera de manera analítica, después utilizaron el método de relajación para estimar los parámetros $\mathrm{k}_{\mathrm{e}} \mathrm{y} / \mathrm{o} \mathrm{h}_{\mathrm{w}}$, según sea el caso. El propósito de este método es minimizar una función objetivo, F, como la que se muestra a continuación: 


$$
F=\sum_{1}^{N}\left(T_{\text {exp }}-T_{\text {calc }}\right)^{2}
$$

donde $\mathrm{N}$ es el número de observaciones, $\mathrm{T}_{\exp }$ es la temperatura experimental medida y $\mathrm{T}_{\text {calc }}$ es la temperatura dada por el modelo. A manera de dar una breve explicación, el método de relajación consiste en mantener un parámetro constante mientras varía el otro parámetro hasta encontrar un F mínimo, una vez encontrado, el valor que se mantenía fijo ahora variará mientras que el otro valor se mantiene fijo, hasta que el valor de una diferencia entre dos $\mathrm{F}$ sea menor a 0.01 , llegando a una región de confianza. Una representación de lo anterior mencionado se señala en la Figura 2.5. Cabe señalar que para casos de estudio en donde sólo se estima un parámetro, la superficie de error se reduce a una curva en un plano con las coordenadas $\mathrm{F}$ y $\mathrm{k}_{\mathrm{e}}$, en donde el mejor valor de $\mathrm{k}_{\mathrm{e}}$ corresponde al mínimo de la curva. Por último, los autores propusieron correlaciones para calcular los parámetros efectivos de transferencia de calor.

Hasta este punto, la mayoría de los trabajos de transferencia de calor en lechos empacados utilizaba alguno de los dos modelos propuestos por De Wasch y Froment ${ }^{28}$, en donde a veces se despreciaba el transporte de calor axial o radial, según el caso de estudio, o inclusive se le añadía al modelo un término transitorio para describir experimentos en la transitoriedad. Sin embargo, trabajos comos los de Finlayson ${ }^{58}$ o Lerou y Froment ${ }^{50}$ fueron de los primeros estudios en considerar que era de suma importancia reemplazar el uso de una velocidad uniforme (flujo pistón, G) dentro del lecho y utilizar un campo de velocidad en dirección axial que variara con la posición radial, $\mathrm{v}_{\mathrm{z}}(\mathrm{r})$. La idea surgió debido a que en estudios experimentales de la literatura se percataron que en la región cercana a la pared, el gradiente de temperaturas se incrementaba más que en la región central del lecho, a lo que se le atribuyó este efecto a la existencia de un perfil radial de velocidad axial. Esto pone en duda el uso de las correlaciones reportadas hasta esa fecha, las cuales consideraban una velocidad uniforme. 


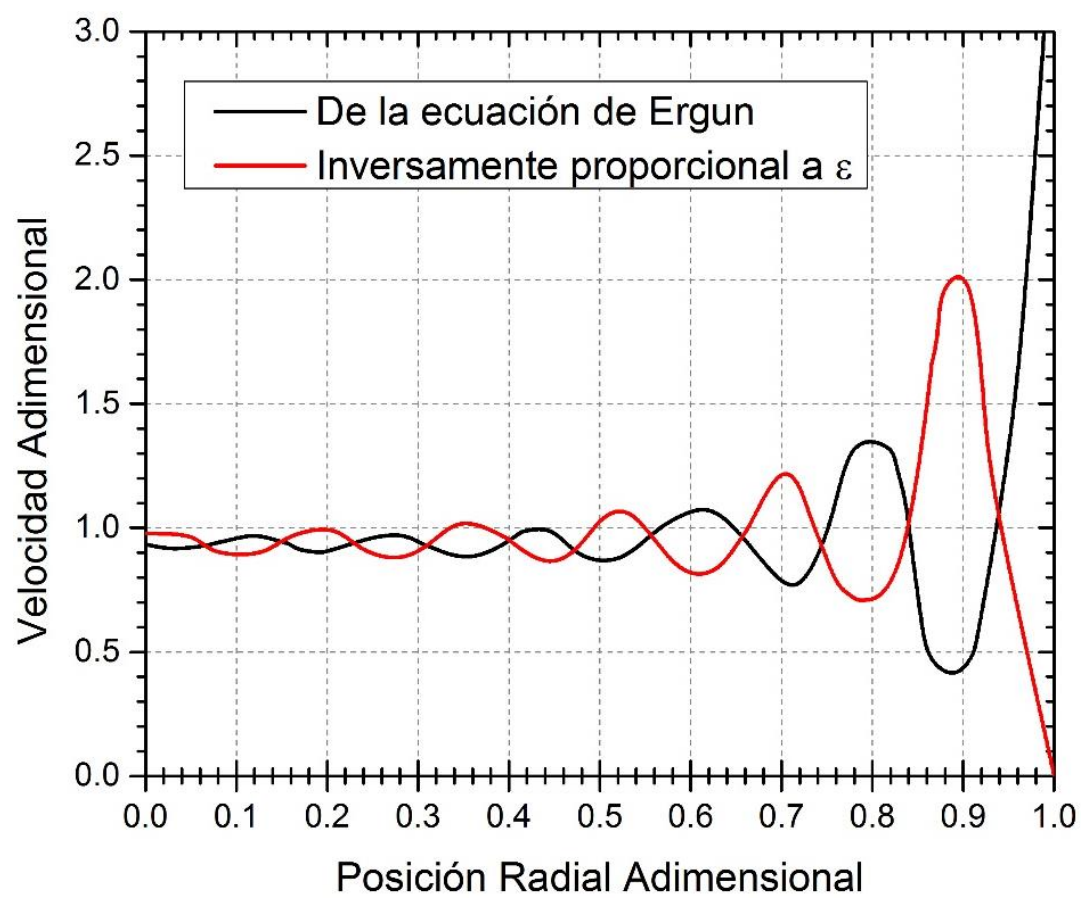

Figura 2.9. Propuestas de distribución de velocidad axial en función del radio de Lerou y Froment ${ }^{50}$

Para ejemplificar lo anterior, el estudio de Lerou y Froment ${ }^{50}$ fue de gran ayuda, su sistema experimental fue similar al usado por De Wasch y Froment ${ }^{28}$ que mostró gradientes de temperaturas altos en la región de la pared. Mientras que para medir los perfiles de velocidad en función del radio, emplearon un anemómetro a la salida del lecho. Las observaciones experimentales del campo de velocidad en dirección axial mostraron un incremento en la velocidad en la región cercana a la pared, en donde, justamente al ubicarse en la pared, la velocidad caía a cero. A pesar de dichos datos experimentales, el perfil de velocidad observado no tenía un parecido a los perfiles de fracción vacía que se habían obtenido en la literatura. Por lo que se optó por desarrollar dos posible distribuciones del campo de velocidad dentro del lecho, mostradas en la figura 2.9.

La aproximación derivada de la ecuación de Ergun no describía el comportamiento en la pared del lecho, por lo que la segunda aproximación tomó en cuenta que la velocidad debía de alcanzar su máximo cuando la fracción vacía era máxima, respetando que la velocidad en la pared estrictamente debía ser cero. 
Este perfil de velocidad fue acoplado a la ecuación de energía, sustituyendo el término del flujo pistón, y los resultados mostraron una gran diferencia en los perfiles de temperatura, ver figura 2.10

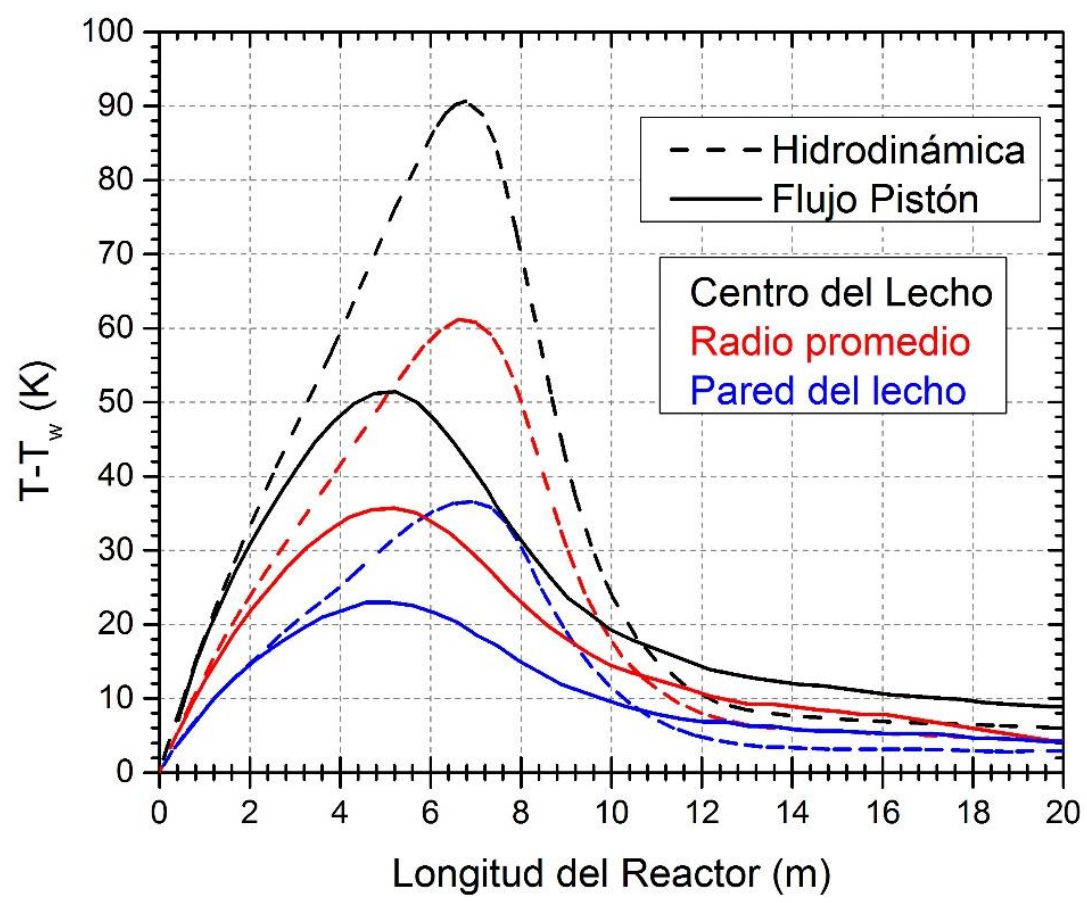

Figura 2.10. Perfil de temperaturas en un lecho empacado, modelo que considera la hidrodinámica (---), modelo con flujo pistón (-).

En esta figura se observa el impacto de usar o no la hidrodinámica en los modelos de la transferencia de calor en los lechos empacados, por lo que estudios posteriores fueron tomando en cuenta el campo de velocidad dentro del lecho para acoplarlo al modelo de transferencia de calor.

Al observar el efecto de considerar una no uniformidad de la velocidad dentro del lecho, varios autores sugerían que la conductividad térmica efectiva radial también debería de estar en función de la posición radial. ${ }^{19-21,23,49,58,59}$ Delmas y Froment ${ }^{59}$ realizaron esto mediante una correlación de estudios anteriores ${ }^{28}$, que consideraba la contribución estática y dinámica, donde sólo sustituyeron la velocidad por la velocidad axial pseudo local en su posición radial, obteniendo un perfil similar al de la fracción vacía y velocidad axial en el lecho, el cual aparenta ser una opción adecuada para describir la transferencia de calor en la región cercana a la pared. 
Ya bastante se ha estudiado en la transferencia de calor en lechos empacados, sin embargo, son muy escasos los estudios que no consideran la pseudohomogeneidad en el modelado del sistema, pues la mayoría considera un equilibrio térmico entre ambas fases, incluso sin haber realizado una medición experimental adecuada en ambas fases. Wen y Ding ${ }^{15}$ resaltan la importancia de lo anterior, realizando un estudio experimental cuya medición de temperaturas fue realizada en ambas fases dentro del lecho, en dirección axial y radial, debido a que el modelado se realiza en estas dos coordenadas.

La diferencia en número entre trabajos que consideran pseudohomogeneidad y pseudoheterogeneidad, es parecida a los trabajos que consideran el estado estacionario y el estado transitorio. Los pocos trabajos que existen que estudian la transitoriedad son, en su mayoría, experimentales ${ }^{15,53,54}$, siendo casi nulos aquellos trabajos que tratan de describir mediante un modelo transitorio los datos experimentales, donde en su mayoría, no se describe satisfactoriamente la transitoriedad ${ }^{29,60}$. Hasta donde se tiene conocimiento, los trabajos teóricos sólo describen la transitoriedad cuando consideran convección libre o conducción en un lecho empacado ${ }^{55-57}$, y sólo el trabajo de Jorge et al. ${ }^{31}$ ha considerado un mecanismo de transporte convectivo, sin embargo, en este trabajo se estima un parámetro que depende de propiedades físicas y termofísicas del sistema, por lo que se cuestiona fuertemente su validez, a pesar de sí describir los datos experimentales mencionados en el trabajo.

Por último, otra opción para describir la transferencia de calor en este tipo de sistemas es el empleo de software de CFD (Computational Fluid Dynamics), la gran desventaja que se tiene al usar este tipo de herramientas es la falta de recursos computacionales que existen hoy en día para poder describir procesos industriales, sin embargo, CFD ha ayudado a proponer nuevas aproximaciones y entender mejor sistemas a escala laboratorio. Un ejemplo de lo anterior mencionado son los trabajos realizados por Dixon ${ }^{41}$ y Benham et al. ${ }^{42}$, en donde proponen que el empleo de una velocidad en dirección axial y radial puede contribuir a una mejor caracterización del transporte convectivo de calor. En sus estudios, se alcanza a ver que la velocidad radial puede tener un valor del 10\% hasta el $30 \%$ de la velocidad axial según su ubicación. 
Capítulo III - "Estudio de la transferencia de calor en el lecho empacado"

\section{$\underline{\text { 3.1 Sistema experimental }}$}

Con el objetivo de emplear la información obtenida de la caracterización de la transferencia de calor de un lecho empacado con relación $d_{t} / d_{p}$ menor a 10 para un reactor de lecho empacado multitubular, en esta sección se presentan los sistemas experimentales de estudio. Es importante señalar que se hace la suposición de que el comportamiento de un tubo empacado es muy similar a los demás tubos empacados dentro de un reactor multitubular. Los datos del campo de temperatura dentro del lecho empacado fueron tomados de estudios previos del Grupo de Procesos de Transporte y Reacción en Sistemas Multifásicos de la UAM-I. ${ }^{4,5}$

A continuación se explican brevemente dichos sistemas experimentales y las condiciones de operación de los mismos.

\subsubsection{Lecho quasi-adiabático}

El primer sistema experimental se conoce como el lecho quasi-adiabático, ver Figura 3.1, este lecho consiste en aislar la transferencia de calor en las paredes mediante fibra de vidrio en la parte externa del lecho, la fuente de calor, una lámpara de luz infrarroja de $250 \mathrm{~W}$, para la cual se registró que en la parte superior del lecho inciden desde $0.0460 \mathrm{~W}$ hasta $1.3128 \mathrm{~W}$ según el flujo empleado, es colocada en la parte superior del lecho, y se hace fluir un flujo de aire por la parte inferior del lecho. Las mediciones de temperatura dentro del lecho fueron tomadas por termopares tipo $\mathrm{K}$ (Chromel-Alumel), de $0.5 \mathrm{~mm}$ de diámetro interno y $0.7 \mathrm{~mm}$ de diámetro externo, a diez diferentes posiciones axiales no equidistantes, en el centro del lecho. El empaque consiste en un catalizador de pentóxido de vanadio $\left(\mathrm{V}_{2} \mathrm{O}_{5}\right)$ depositado externamente en un núcleo de dióxido de titanio $\left(\mathrm{TiO}_{2}\right)$, con una conductividad térmica de

$0.1047 \mathrm{~W} / \mathrm{m} \mathrm{K}$, densidad de $2000.0 \mathrm{~kg} / \mathrm{m}^{3}$, y un calor específico de $104.7 \mathrm{~J} / \mathrm{kg} \mathrm{K}{ }^{18,61} \mathrm{El}$ lecho mide $41.86 \mathrm{~cm}$ de largo, las paredes del tubo son de acero inoxidable 316, y su relación diámetro de tubo a partícula $\left(\mathrm{d}_{\mathrm{t}} / \mathrm{d}_{\mathrm{p}}\right)$ es de 3.175. 


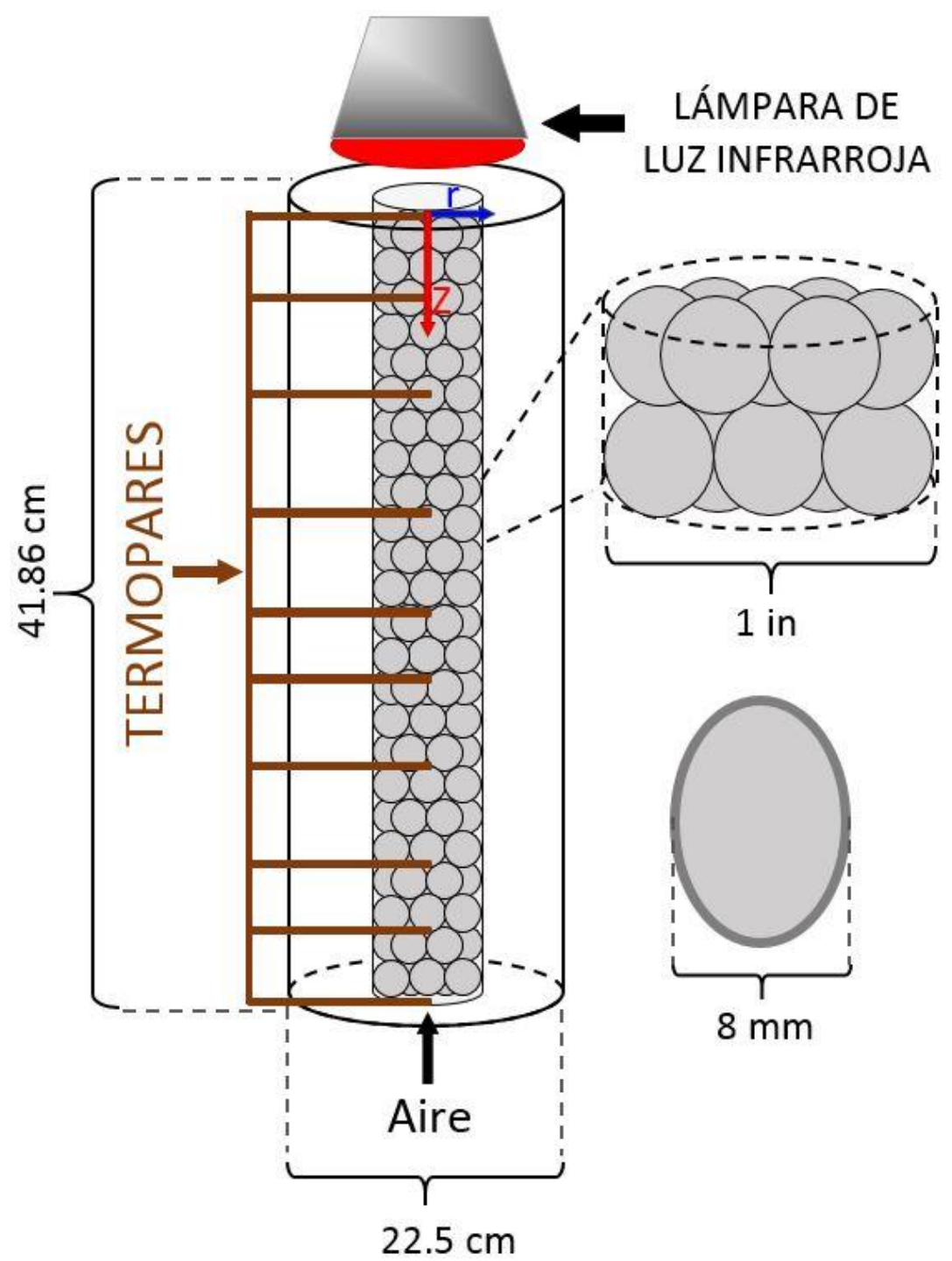

Figura 3.1. Sistema experimental, lecho quasi-adiabático.

\subsubsection{Lecho no isotérmico y no adiabático}

El segundo sistema experimental consiste en el mismo lecho, con la excepción de que ahora la fuente de calor es una resistencia eléctrica en las paredes, y el flujo del aire se alimenta por la parte superior del lecho, ver Figura 3.2; el empaque, termopares y dimensiones del lecho son los mismos que los del sistema quasi-adiabático. Cabe señalar que los termopares ahora capturaron el campo de temperatura a cinco diferentes posiciones radiales en cada posición axial, para esto, se realizó el experimento en donde los termopares midieron la temperatura en el centro del lecho, posteriormente, se volvió a hacer el experimento ahora con los termopares a una distancia $\mathrm{r}$ del centro del lecho, esto se realizó a 5 posiciones radiales. 


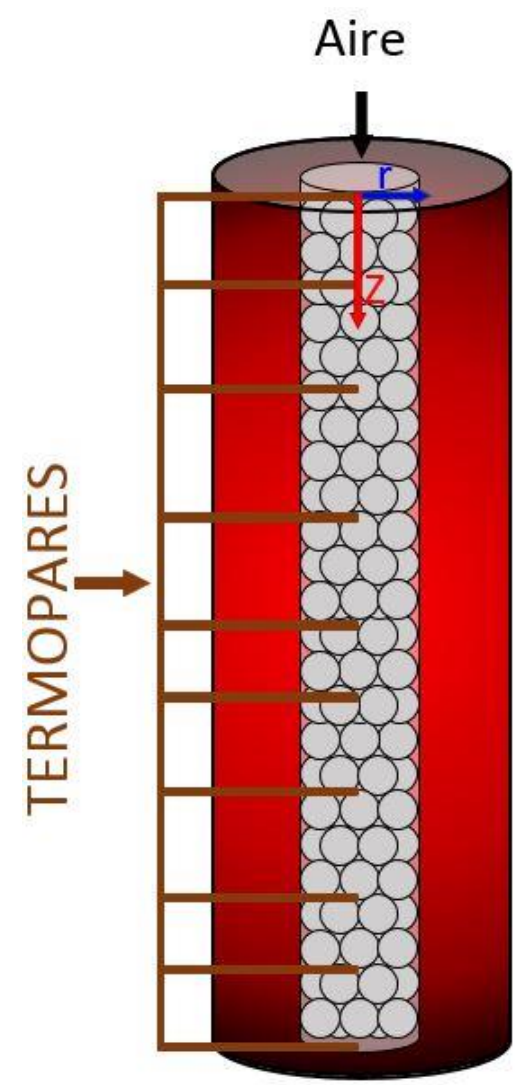

Figura 3.2. Sistema experimental, lecho no isotérmico no adiabático

\subsection{Experimentos de transferencia de calor}

En este trabajo se toman en cuenta tres casos experimentales que se llevaron a cabo en los sistemas experimentales mencionados anteriormente ${ }^{4,5}$, con la finalidad de obtener el campo de temperatura dentro de los lechos a diferentes condiciones de flujo y calentamiento, los cuales se describen a continuación.

\subsubsection{Caso 1 - Estados estacionarios en el lecho quasi-adiabático}

En este caso, se utiliza el lecho quasi-adiabático, el cual se calienta con una lámpara de luz infrarroja de $250 \mathrm{~W}$ por la parte superior del lecho. El calentamiento del lecho dura aproximadamente 2 horas para llegar a su estado estacionario. Una vez que se encuentre en estado estacionario, se hace fluir por la parte inferior aire a temperatura ambiente $\left(\sim 20-24^{\circ} \mathrm{C}\right)$ hasta llegar a un estado estacionario en el lecho. Es importante reiterar que la medición de las temperaturas son realizadas por 10 termopares no equidistantes en el centro del lecho; los flujos empleados en este caso de estudio son descritos en la tabla 3.1. 


\begin{tabular}{|c|c|c|}
\hline \multicolumn{3}{|c|}{$\begin{array}{c}\text { Tabla 3.1. Condiciones de } \\
\text { experimentación del caso } 1\end{array}$} \\
\hline $\begin{array}{c}\text { Flujo de aire } \\
(\mathbf{L} / \mathbf{m i n})\end{array}$ & $\begin{array}{c}\text { Flux másico } \\
\left(\mathbf{g} / \mathbf{c m}^{2} \mathbf{m i n}\right)\end{array}$ & $\mathbf{R e}_{\mathbf{p}}$ \\
\hline 0 & 0 & 0 \\
\hline 5 & 1.2196 & 93.70 \\
\hline 10 & 2.4393 & 187.40 \\
\hline 20 & 4.8786 & 374.80 \\
\hline 30 & 7.3179 & 562.21 \\
\hline 40 & 9.7572 & 749.61 \\
\hline
\end{tabular}

\subsubsection{Caso 2 - Estados transitorios en el lecho quasi-adiabático, experimento dinámico.}

En el lecho quasi-adiabático se realizó un experimento de pulsos de flujo de fluido en condiciones transientes; Los datos de los flujos empleados se encuentran en la Tabla 3.2.

\begin{tabular}{|c|c|c|}
\hline \multicolumn{3}{|c|}{$\begin{array}{c}\text { Tabla 3.2. Condiciones de } \\
\text { experimentación del caso } 2\end{array}$} \\
\hline $\begin{array}{c}\text { Flujo de aire } \\
(\mathbf{L} / \mathbf{m i n})\end{array}$ & $\begin{array}{c}\text { Flux másico } \\
\left(\mathbf{g} / \mathbf{c m}^{2} \text { min }\right)\end{array}$ & $\mathbf{R e}_{\mathbf{p}}$ \\
\hline 9 & 2.1953 & 168.66 \\
\hline 15.4 & 3.7565 & 288.60 \\
\hline 8.65 & 2.1099 & 162.10 \\
\hline 16.55 & 4.0370 & 310.15 \\
\hline 8.86 & 2.1612 & 166.04 \\
\hline 16.5 & 4.0248 & 309.21 \\
\hline
\end{tabular}

El objetivo del experimento es obtener la distribución de temperaturas en la transitoriedad de un punto dentro del lecho; en este caso se tomó la lectura en la posición z=0. Para realizar el experimento, primero se debe de obtener el estado estacionario a un flujo dado, una vez alcanzado este estado, se varía el flujo, aumentando o disminuyendo según se deseé el 
aumento o disminución de temperatura, y se mantiene fijo hasta volver a alcanzar otro estado estacionario, el proceso se repite variando el flujo.

\subsubsection{Caso 3 - Estados estacionarios en el lecho no isotérmico no adiabático.}

En este caso se obtiene el campo de temperaturas en el estado estacionario del lecho no isotérmico no adiabático a $\mathrm{Re}_{\mathrm{p}}$ de $163.36,81.67$ y 40.83 en 10 posiciones axiales, en donde cada posición axial a su vez registra la temperatura a 5 posiciones radiales. A diferencia del caso 1, el calentamiento del lecho se realiza por la pared mediante una resistencia eléctrica, como se mencionó anteriormente, ver Figura 3.2. El procedimiento para obtener las temperaturas al flujo empleado es similar al caso 1, primero se calienta el lecho en ausencia de un flujo, una vez alcanzado el estado estacionario se hace fluir un fluido, aire, mediante la parte superior del lecho, y se registra el estado estacionario del lecho.

\subsection{Modelos de transferencia de calor en el lecho empacado}

En este trabajo se toman en cuenta 3 modelos de transferencia de calor para el lecho quasiadiabático y 1 para el lecho con calentamiento en la pared. Los modelos se aplican a tres casos experimentales mencionados anteriormente, según correspondan.

\subsubsection{Modelo adiabático pseudo-homogéneo en estado estacionario}

Se propone un modelo de transferencia de calor que capture la información obtenida del caso experimental 1, considerando un equilibrio térmico en ambas fases del sistema.

El modelo propuesto y sus condiciones de frontera son los siguientes:

$$
\begin{gathered}
\rho_{f} C_{p_{f}} v_{z} \frac{\partial T}{\partial z}=k_{e f f, z} \frac{\partial^{2} T}{\partial z^{2}} \\
\text { en } z=0 ; \quad k_{e f f, z} \frac{\partial T}{\partial z}=q \\
\text { en } z=L ; \quad T=T_{\text {in }}
\end{gathered}
$$

Cabe señalar que la descripción de los gradientes de temperatura radiales se omite, debido a que no hay gradientes radiales apreciables en este tipo de lechos con paredes quasiadiabáticas, por lo que el uso de un flujo pistón es adecuado. $\mathrm{La} \mathrm{T}_{\mathrm{in}}$ en $\mathrm{z}=0$ es la temperatura con la que entra el aire, debido a que en esa posición se registró una temperatura ambiente 
constante, y q es el flux de calor que influyó en la parte superior del lecho, cabe señalar que el flujo de calor va de $\mathrm{z}=0$ a $\mathrm{z}=\mathrm{L}$, como se muestra en la Figura 3.1. El modelo fue resuelto mediante la discretización de los términos diferenciales con el método numérico de colocación ortogonal (CO) utilizando 15 puntos de colocación, el sistema de ecuaciones algebraicas fue resuelto mediante el método de sustitución, y para la estimación del parámetro keff,z se utilizó el método de Mínimos cuadrados con el algoritmo de LevenbergMarquardt (MC-LM), en donde se emplearon las 10 posiciones axiales obtenidas experimentalmente para cada perfil de temperatura, es decir, para cada $\mathrm{Re}_{\mathrm{p}}$. La solución del método de CO fue comparado con la solución analítica de este modelo para comparar que no existieran diferencias apreciables entre ambas soluciones, así como también se escogió el mínimo de puntos de colocación requeridos para obtener una descripción adecuada de los perfiles de temperatura. Lo anterior, para tener confianza en los resultados del método numérico, el cual será empleado para resolver modelos más complejos.

\subsubsection{Modelo adiabático pseudo-homogéneo en estado transitorio}

Asumiendo nuevamente un equilibrio térmico entre ambas fases del sistema, ahora se propone un modelo de transferencia de calor que capture la información en la transitoriedad del lecho.

El modelo propuesto y sus condiciones de frontera e iniciales se presentan a continuación:

$$
\begin{gathered}
\rho C_{p} \frac{\partial T}{\partial t}+\rho_{f} C_{p_{f}} v_{z} \frac{\partial T}{\partial z}=k_{e f f, z} \frac{\partial^{2} T}{\partial z^{2}} \\
\text { a } t=0 ; \quad T(z)=T_{s s}(z) \\
\text { en } z=0 ; \quad k_{e f f, z} \frac{\partial T}{\partial z}=q \\
\text { en } z=L ; \quad T(t)=T_{\text {in }}(t)
\end{gathered}
$$

donde cabe señalar que $\rho C_{p}$ es la suma de las capacidades caloríficas volumétricas de la fase fluida y sólido, $\mathrm{T}_{\mathrm{ss}}$ es la temperatura de un flujo de aire en estado estacionario a un tiempo 0 en una posición z, y q es el flux de calor que influye en la parte superior del lecho y fluye hacia la parte inferior del lecho. Este modelo sirve para describir los casos experimentales 1 y 2 . 
El modelo fue resuelto mediante la discretización de los términos diferenciales con el método numérico de $\mathrm{CO}$ utilizando 15 puntos de colocación y 10 posiciones axiales experimentales, y el sistema de ecuaciones diferenciales fue resuelto utilizando el método de Runge-Kutta de cuarto orden. Para la estimación del parámetro $\mathrm{k}_{\mathrm{eff}, \mathrm{z}}$, también fue empleado el método de MCLM.

\subsubsection{Modelo adiabático pseudo-heterogéneo en estado transitorio}

Para capturar la información de la transferencia de calor en la fase sólida y fluida del lecho empacado, en estado transitorio, se propone el siguiente modelo y sus respectivas condiciones de frontera para la fase sólida

$$
\begin{gathered}
\rho_{s} C p_{s} \frac{\partial T_{s}}{\partial t}=k_{s, e f f, z} \frac{\partial^{2} T_{s}}{\partial z^{2}}+h_{g} a_{s}\left(T_{f}-T_{s}\right) \\
\text { a } t=0 ; \quad T(z)=T_{s s}(z) \\
\text { en } z=0 ; \quad k_{s, e f f, z} \frac{\partial T_{s}}{\partial z}=q \\
\text { en } z=L ; \quad T(t)=T_{\text {in }}(t)
\end{gathered}
$$

Y para la fase fluida

$$
\begin{gathered}
\rho_{f} C p_{f} \frac{\partial T_{f}}{\partial t}+\rho_{f} C p_{f} v_{z} \frac{\partial T_{f}}{\partial z}=k_{f, e f f, z} \frac{\partial^{2} T_{f}}{\partial z^{2}}-h_{g} a_{s}\left(T_{f}-T_{s}\right) \\
\text { a } t=0 ; \quad T(z)=T_{S S}(z) \\
\text { en } z=0 ; \quad k_{f, e f f, z} \frac{\partial T_{f}}{\partial z}=q \\
\text { en } z=L ; \quad T(t)=T_{\text {in }}(t)
\end{gathered}
$$

En donde los subíndices s y f representan la fase sólida y fluida, respectivamente, el parámetro $h_{\mathrm{g}}$ es el coeficiente de transferencia de calor interfacial sólido-fluido, y as es el área específica por unidad de volumen de una partícula de $\mathrm{V}_{2} \mathrm{O}_{5} / \mathrm{TiO}_{2}$. Ambos modelos fueron resueltos por $\mathrm{CO}$ con 15 puntos de colocación y MC-LM, así como también el uso de 10 posiciones axiales experimentales, estos modelos aplican para resolver el caso 1 y 2 de estudio. 


\subsubsection{Modelo no isotérmico no adiabático pseudo-homogéneo en estado transitorio}

Para el lecho con calentamiento en la pared se propone un modelo en estado transitorio que considere la transferencia de calor en la dirección axial y radial, el cual se presenta a continuación junto con sus respectivas condiciones de frontera.

$$
\begin{gathered}
\rho C p \frac{\partial T}{\partial t}+\rho_{f} C p_{f} v_{z} \frac{\partial T}{\partial z}=k_{e f f, z} \frac{\partial^{2} T}{\partial z^{2}}+k_{e f f, r}\left(\frac{\partial^{2} T}{\partial r^{2}}+\frac{1}{r} \frac{\partial T}{\partial r}\right) \\
\text { en } t=0 ; \quad T(r, z)=T_{s s}(r, z) \\
\text { en } z=0 ; v_{z} \rho_{f} C p_{f}\left(T_{i n}(t, r)-T(t, r)\right)=k_{e f f, z} \frac{d T}{d z} \\
\text { en } z=L ; \quad \frac{d T}{d z}=0 \\
\text { en } r=0 ; \quad \frac{d T}{d r}=0 \\
\text { en } r=R_{t} ;-k_{e f f, r} \frac{d T}{d r}=h_{w}\left(T(t, z)-T_{w}(t, z)\right) \\
\text { en } r=R_{t} ; T(t, z)=T_{w}(t, z)
\end{gathered}
$$

En este modelo, la condición de frontera 3.3.3g puede emplearse si el modelo considera la aproximación de la capa límite, en la cual, el uso de la hidrodinámica implica que el parámetro keff,r captura todos los mecanismos de transferencia de calor radiales en el lecho, y en caso de utilizar flujo pistón, se utilizar la condición de frontera 3.3.3f $\mathrm{f}^{5}$. Para resolver el modelo se utilizó el método de $\mathrm{CO}$ con 20 puntos de colocación axial y 20 puntos radiales, el sistema de ecuaciones diferenciales fue resuelto con el método de Runge-Kutta de cuarto orden y para la estimación de los parámetros efectivos se utilizó el método de MC-LM.

\subsection{Modelo de Hidrodinámica en el lecho empacado.}

La ecuación de Navier-Stokes-Darcy-Forchheimer (NS-DF) es comúnmente utilizada para acoplar el campo de velocidad dentro del lecho a la ecuación de transferencia de energía, véase Ec.3.4.1.

$$
\rho_{f}\left[\varepsilon \frac{\partial v_{z}}{\partial t}+\varepsilon^{2} v_{z} \frac{\partial v_{z}}{\partial z}\right]=-\varepsilon \frac{\partial p_{z}}{\partial z}+\mu_{e f f} \nabla^{2} \varepsilon v_{z}-\left(\frac{\mu_{f}}{K} \varepsilon v_{z}+\frac{\rho_{f}}{K_{z}} \varepsilon^{2} v_{z}^{2}\right)+\varepsilon \rho_{f} g_{z}
$$


Sin embargo, recientemente se ha desarrollado una aproximación más simple para describir dicho campo de velocidad. Las ventajas que brinda esta nueva aproximación son debidas a que su solución es pseudo-analítica, mientras que NS-DF requiere de una solución numérica para acoplar después a la descripción de la transferencia de calor en el lecho, lo cual repercute en los tiempos de cómputo empleados para la solución de los modelos, así como la estimación de los parámetros efectivos del mismo. Una breve descripción de esta nueva aproximación se da a continuación.

Esta aproximación, propuesta por Aparicio et al. ${ }^{3}$, para la predicción del campo de velocidad dentro del lecho empacado, se fundamenta en el hecho de que no se tiene claro el uso de la ecuación de NS-DF para describir los perfiles de velocidad en el lecho, debido a que el término de Navier-Stokes sólo debe de ser válido en la región cercana a la pared, en donde las interacciones fluido-fluido son dominantes, mientras que el término de DarcyForchheimer sólo debe de ser válido en la región central del lecho, debido a que las interacciones sólido-fluido son dominantes, como se muestran en la figura 3.3. Esta aproximación es validada para relaciones de $d_{t} / d_{p}$ entre 3 y 6, y $\operatorname{Re}_{p}$ entre 500 y 1400 , con respecto a las predicciones de la solución numérica del modelo de NS-DF, y a observaciones experimentales de velocidad.

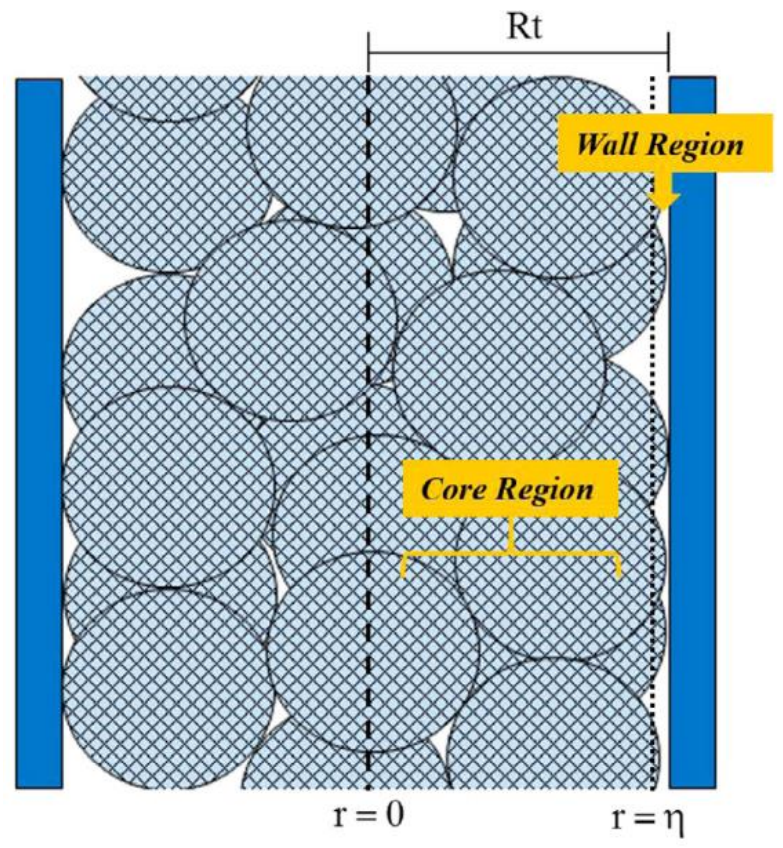

Figura 3.3. Ilustración de la región central y de pared en un lecho empacado ${ }^{3}$. 
A continuación se muestran los modelos desarrollados para describir la velocidad en dirección axial para la región central:

$$
0=-\varepsilon \frac{\partial p_{z}}{\partial z}-\left[\frac{\mu_{f}}{K} \varepsilon v_{z}^{C R}+\frac{\rho_{f}}{K_{z}} \varepsilon^{2}\left(v_{z}^{C R}\right)^{2}\right]+\varepsilon \rho_{f} g_{z}
$$

Y de pared:

$$
\rho_{f} \varepsilon^{2} v_{z}^{W R} \frac{\partial v_{z}^{W R}}{\partial z}=-\varepsilon \frac{\partial p_{z}}{\partial z}+\frac{1}{r} \frac{\partial}{\partial r}\left(\mu_{e f f} r \frac{\partial \varepsilon v_{z}^{W R}}{\partial r}\right)
$$

En donde la soluciones para dichos modelos, respectivamente, son:

$$
\begin{gathered}
v_{z}^{C R}=\frac{-1+\sqrt{1+\lambda}}{\varphi} \\
v_{z}^{W R}=\left.\left[\frac{\left(1+\theta_{t}\right) \ln \left(1+\theta_{t} x\right)-\theta_{t} x}{\left(1+\theta_{t}\right) \ln \left(1+\theta_{t}\right)-\theta_{t}}\right] v_{z}^{C R}\right|_{x=1}
\end{gathered}
$$

Cabe señalar que los valores de $\lambda$ y $\varphi$, para la solución del modelo en la región central son:

$$
\begin{gathered}
\lambda=4 \varepsilon \frac{\rho_{f}}{\mu_{f}^{2}}\left(\frac{K^{2}}{K_{z}}\right)\left(\frac{\Delta p_{z}}{L}+\rho_{f} g_{z}\right) \\
\varphi=2 \varepsilon \frac{\rho_{f}}{\mu_{f}}\left(\frac{K}{K_{z}}\right)
\end{gathered}
$$

Y las definiciones de $\theta_{\mathrm{t}} \mathrm{y}$ x, para la solución del modelo en la región de la pared son:

$$
\begin{gathered}
\theta_{t}=\frac{\mu_{t}}{\mu_{f}} \\
x=\frac{\left(R_{t}-r\right)}{\delta}
\end{gathered}
$$

En donde $\delta$ es un pseudo espesor de la capa límite y $\mu_{\mathrm{t}}$ es la viscosidad turbulenta.

Dado que el caso de estudio cumple con las consideraciones de esta aproximación, esta es utilizada en el término convectivo de los modelos de transferencia de calor que consideran la hidrodinámica. Para un mejor entendimiento de esta aproximación, se puede consultar el trabajo de Aparicio et al. ${ }^{3}$ 


\section{Capítulo IV - Resultados y discusión}

\subsection{Lecho quasi-adiabático}

En esta sección se presentan y analizan los resultados obtenidos para el lecho quasiadiabático. Primero se muestra la caracterización de la transferencia de calor para el estado estacionario, con los modelos pseudo-homogéneo y pseudo-heterogéneo, después se realiza la misma acción pero para el experimento dinámico. Por último, se evalúa el coeficiente de transferencia de calor interfacial en el experimento dinámico.

\subsubsection{Parámetros térmicos efectivos axiales en estado estacionario}

En este punto se caracteriza la conductividad térmica efectiva axial $\left(\mathrm{k}_{\mathrm{eff}, \mathrm{z}}\right)$ utilizando dos modelos, uno pseudo-homogéneo y uno pseudo-heterogéneo, y utilizando los datos experimentales del caso 1 , en donde se registran a seis diferentes flujos de alimentación los perfiles de temperatura en el estado estacionario. Esto se realiza con el objetivo de analizar el papel del sólido en este mecanismo de transferencia de calor.

\section{Modelo pseudo-homogéneo}

En la Figura 4.1 se presentan los perfiles de temperaturas axiales ajustados con el modelo pseudo-homogéneo en estado estacionario, Ecuación 3.3.1, a diferentes $\operatorname{Re}_{\mathrm{p}}$ con regiones de $\pm 2.5 \mathrm{~K}$ de error del valor experimental. Las respectivas $\mathrm{k}_{\mathrm{eff}, \mathrm{z}}$ estimadas se muestran en la Tabla 4.1 junto con sus valores estadísticos: intervalos de Confianza del 95\% (I.C. 95\%), y el coeficiente de correlación entre los datos experimentales y el modelo $\left(\mathrm{R}^{2}\right)$.

\begin{tabular}{|c|c|c|c|}
\hline \multicolumn{4}{|c|}{ Tabla 4.1. Keff,z en el lecho quasi-adiabático, modelo } \\
pseudo-homogéneo
\end{tabular}




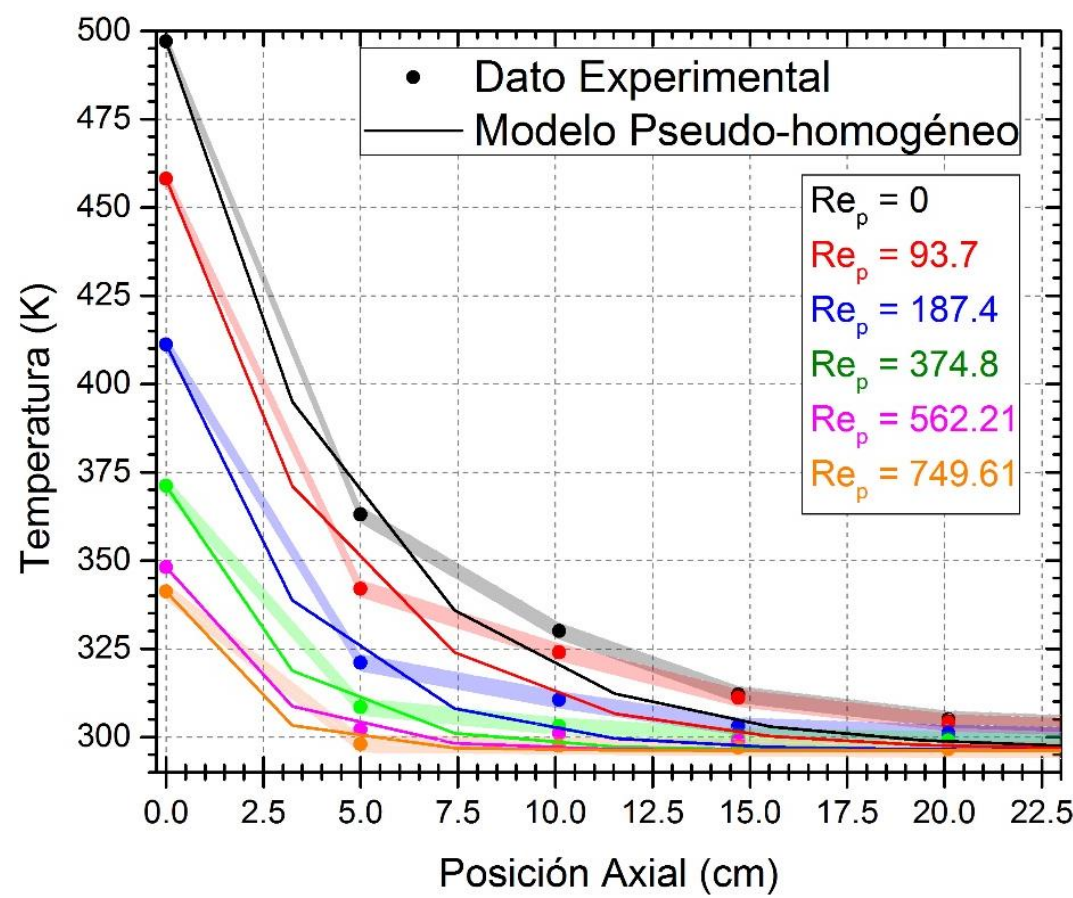

Figura 4.1. Perfiles axiales de temperatura a diferentes $\mathrm{Re}_{\mathrm{p}}$ en el lecho quasi-adiabático, modelo pseudo-homogéneo, el sombreado indica el $\pm 2.5 \mathrm{~K}$ de error.

El I.C. de $95 \%$ de las $\mathrm{k}_{\text {eff,z }}$ estimadas se ubican entre un $\pm 15 \%$ y $\pm 29 \%$ del valor estimado; estos rangos son generalmente reportados en la literatura. ${ }^{3,25,26,28}$ Los coeficientes de correlación, $\mathrm{R}^{2}$, obtenidos en la estimación de las $\mathrm{k}_{\mathrm{eff}, \mathrm{z}}$ tiene valores por encima de 0.99 , los cuales, por lo general, han sido encontrados en la literatura para este coeficiente. Las temperaturas predichas por el modelo pseudo-homogéneo están dentro del margen de error del valor experimental, $\pm 2.5 \mathrm{~K}$.

El lecho presenta gradientes de temperatura axiales despreciables después de los $20 \mathrm{~cm}$, en donde esta es igual a la temperatura a la que se alimenta el fluido al sistema. De acuerdo a los fundamentos, el mayor gradiente de temperatura se obtiene cuando el $\mathrm{Re}_{\mathrm{p}}$ es cero. La $\mathrm{K}_{\mathrm{eff}, \mathrm{z}}$ que se estima a estas condiciones caracteriza la transferencia de calor bajo condiciones estáticas, capturando los mecanismos de transferencia de calor independientes del transporte convectivo del fluido: conducción a través del fluido estático, conducción por contacto entre partículas y la conducción a través del sólido. La cantidad de movimiento por el mecanismo de convección mejora la transferencia de calor en el sistema, por lo que $k_{\text {eff,z }}$ incrementa en función del $\mathrm{Re}_{\mathrm{p}}$. Con las conductividades efectivas estimadas, se generó una correlación para 
este sistema, que a diferencia de las correlaciones propuestas en donde $\mathrm{k}_{\mathrm{eff}, \mathrm{z}}$ varía de manera lineal con $\operatorname{Re}_{\mathrm{p}}{ }^{9,11,12,14-16}$, se tomó en cuenta una correlación similar a la de Metzger ${ }^{13}$, que se presenta a continuación, y que considera dos parámetros que afectan al $\operatorname{Re}_{\mathrm{p}}, \alpha \mathrm{y} \beta$, que esencialmente capturan los efectos hidrodinámicos en la transferencia de calor en dirección axial para este lecho empacado con relación $\mathrm{d}_{\mathrm{t}} / \mathrm{d}_{\mathrm{p}}=3.175$.

$$
k_{e f f, z}=k_{e f f . z}^{0}+\alpha \operatorname{PrR} e_{p}^{\beta}
$$

Los valores de los parámetros $\alpha$ y $\beta$ para el sistema de estudio de este trabajo son 0.3923 y 0.7225, respectivamente. El parámetro $\alpha$ presenta un intervalo de confianza del $95 \%$ de 0.0970-0.6876, mientras que el parámetro $\beta$ tiene un intervalo de confianza del 95\% de 0.5997-0.8453, estos parámetros proporcionan un coeficiente de correlación de 0.9992 con respecto a las $\mathrm{k}_{\mathrm{eff}, \mathrm{z}}$ estimadas.

Para mostrar la importancia de estimar los parámetros efectivos para cada sistema de estudio, en lugar de utilizar un valor de $\mathrm{k}_{\text {eff,z }}$ reportado de correlaciones, la Figura 4.2 presenta la predicción para cuatro $\mathrm{Re}_{\mathrm{p}}$ diferentes utilizando los valores de $\mathrm{k}_{\mathrm{eff}, \mathrm{z}}$ obtenidos de este trabajo, $\mathrm{y}$ aquellos obtenidos de correlaciones reportadas en la literatura ${ }^{13-15}$.

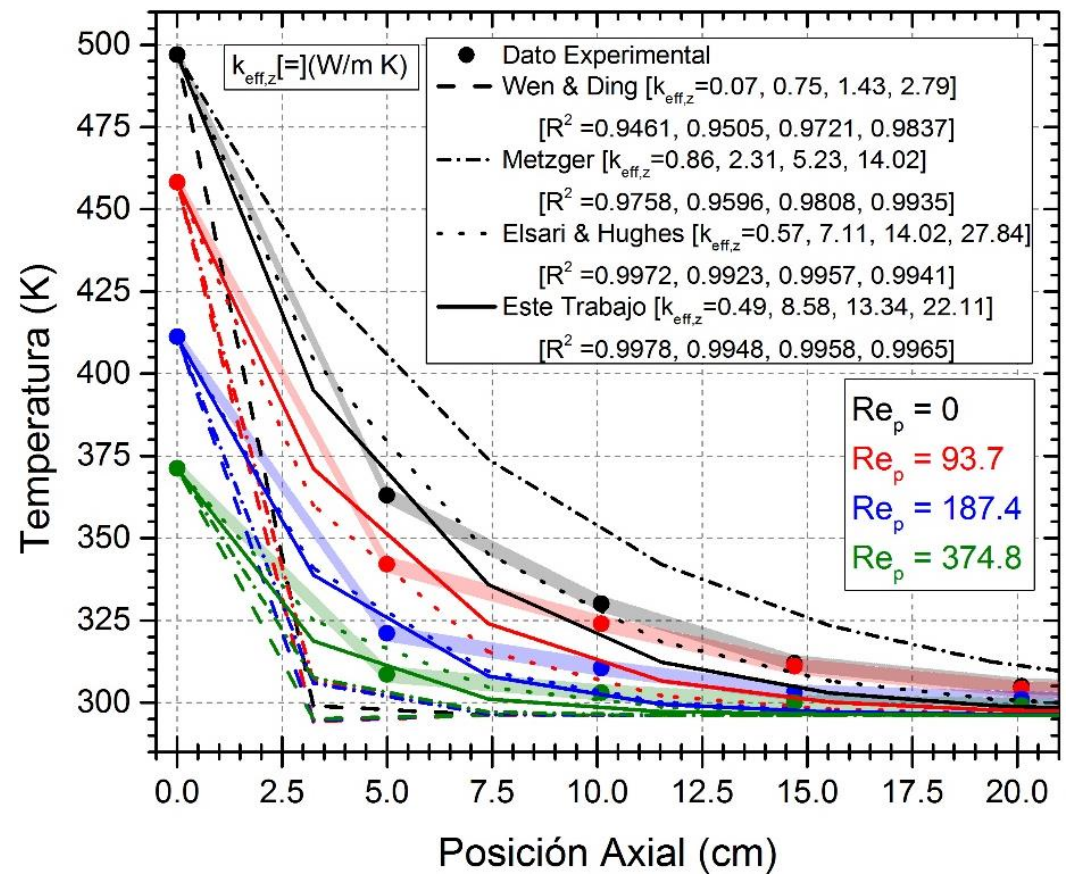

Figura 4.2. Perfiles axiales de temperatura a diferentes $\operatorname{Re}_{\mathrm{p}}$ en el lecho quasi-adiabático, modelo pseudo-homogéneo, distintas correlaciones, el sombreado indica el $\pm 2.5 \mathrm{~K}$ de error. 
Si bien, todas las $k_{\text {eff, } z}$ están en el mismo orden de magnitud, claramente se observa el efecto de la magnitud de este parámetro en la predicción del perfil de temperatura axial, donde , los valores obtenidos en este trabajo son los que mejor describen los perfiles de temperatura observados, ver $\mathrm{R}^{2}$ en la Figura 4.2. Esto en particular tendrá un impacto significativo al momento de utilizar estos parámetros para caracterizar la transferencia de calor en un reactor de lecho empacado a escala industrial ${ }^{2,18,61}$.

\section{Modelo pseudo-heterogéneo}

En la Figura 4.3 se presentan los perfiles de temperaturas axiales obtenidos con el modelo pseudo-heterogéneo en estado estacionario, Ecuación 3.3.3, a diferentes $\mathrm{Re}_{\mathrm{p}}$. La diferencia entre los perfiles de temperatura obtenidos con el modelo pseudo-heterogéneo, tanto de la fase sólida como de la fase fluida, es despreciable. Al comparar estos perfiles de temperatura con los obtenidos con el modelo pseudo-homogéneo, tampoco existe una diferencia apreciable, por lo que, en este caso que considera observaciones de temperatura en estado estacionario, es posible considerar un equilibrio térmico entre ambas fases y utilizar un modelo pseudo-homogéneo para la descripción del lecho, como ya ha sido ampliamente reportado en la literatura. ${ }^{5,8,9,23,28,38,40,50,51}$

Aunado a lo anterior, los parámetros de conductividad axial estimados con el modelo pseudoheterogéneo, tanto para la fase fluida como para la fase sólida son estadísticamente casi iguales, al igual que sus I.C. del 95\%, ver Tabla 4.2. Además, no se presentan diferencias notables de los parámetros estimados del modelo pseudo-homogéneo, así como tampoco su coeficiente de correlación, para cada $\mathrm{Re}_{\mathrm{p}}$, es muy distinto, Tabla 4.1. De esta forma, cuando se usa un modelo pseudo-heterogéneo, ambas fases se caracterizan por tener la misma conductividad térmica efectiva, debido a que por la naturaleza del modelo, que considera al sistema como pseudo-continuo, hace que los mecanismos dependientes y no dependientes del fluido sean capturados en este parámetro, no importando la fase en la que se utilice. Es decir, los mecanismos de transferencia de calor de ambas fase están fuertemente ligados en el parámetro keff,z, los cuales no pueden ser separados mediante la caracterización de la transferencia de calor de ambas fases con dicho parámetro. 


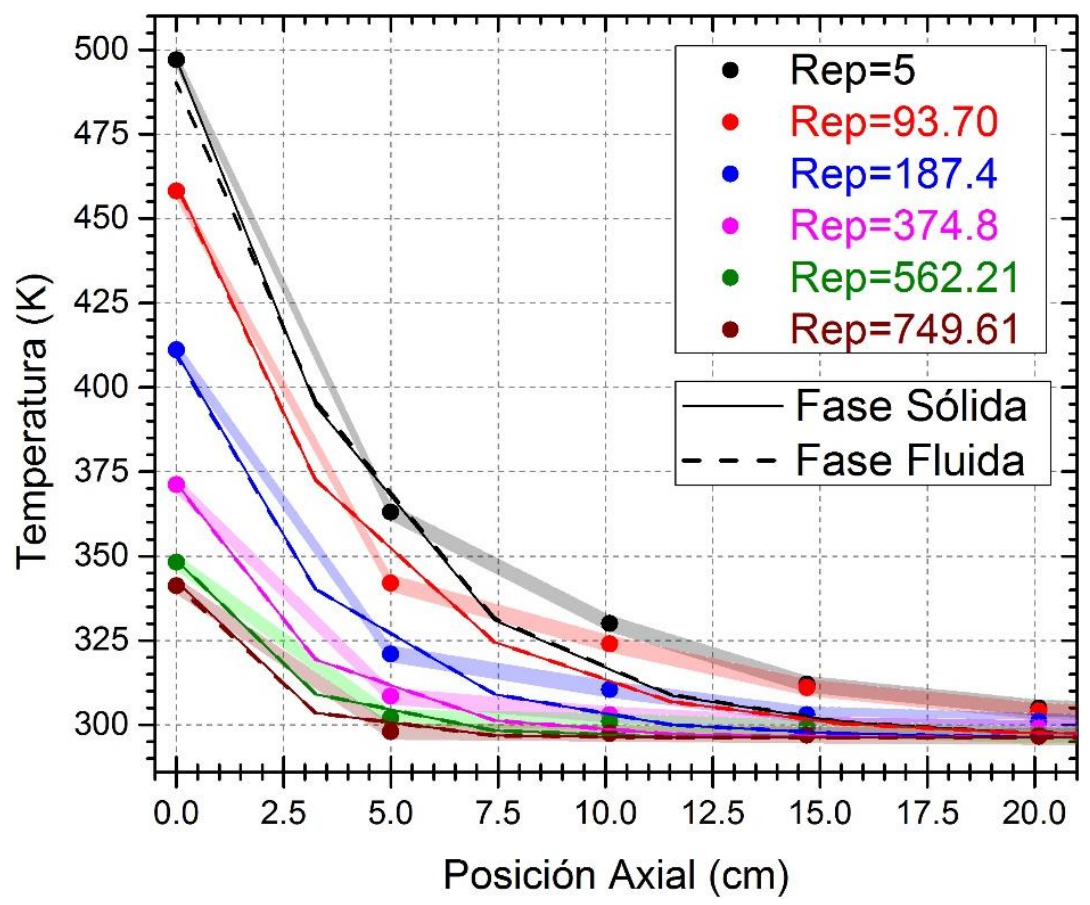

Figura 4.3. Perfiles axiales de temperatura a diferentes $\mathrm{Re}_{\mathrm{p}}$ en el lecho quasi-adiabático, modelo pseudo-heterogéneo, fase sólida (-) y fase fluida (---), región sombreada representan los $\pm 2.5 \mathrm{~K}$ de error.

\begin{tabular}{|c|c|c|c|c|c|c|}
\hline \multicolumn{7}{|c|}{$\begin{array}{r}\text { Tabla 4.2. Parámetros efectivos en el lecho qu } \\
\text { heterogéneo }\end{array}$} \\
\hline $\mathbf{R e}_{\mathbf{p}}$ & $\begin{array}{c}\text { Ks,eff,z } \\
(\mathbf{W} / \mathbf{m} \mathbf{K})\end{array}$ & $\begin{array}{c}\text { I.C. } 95 \% \\
k_{s, e f f, z}\end{array}$ & $\begin{array}{c}\text { kf,eff,z } \\
(\mathrm{W} / \mathrm{m} \mathrm{K})\end{array}$ & $\begin{array}{c}\text { I.C. } 95 \% \\
k_{f, e f f, z}\end{array}$ & $\begin{array}{c}\mathbf{h}_{\mathrm{g}} \\
\left(\mathrm{W} / \mathbf{m}^{2} \mathbf{K}\right)\end{array}$ & $\mathbf{R}^{2}$ \\
\hline 0 & 0.6055 & $0.4543-0.7457$ & 0.2990 & $0.2366-0.3634$ & 13.6246 & 0.9953 \\
\hline 93.70 & 9.7749 & $6.5012-12.2272$ & 8.5451 & $5.6833-11.4069$ & 50.6788 & 0.9946 \\
\hline 187.40 & 14.4210 & $13.2458-15.4988$ & 13.9278 & $12.8026-15.0529$ & 73.7667 & 0.9956 \\
\hline 374.80 & 22.5978 & $16.9137-27.1395$ & 22.3746 & $16.7467-28.0026$ & 108.7616 & 0.9964 \\
\hline 562.21 & 27.9769 & $18.3830-37.7172$ & 28.1541 & $18.4512-37.8571$ & 137.0908 & 0.9956 \\
\hline 749.61 & 35.0365 & $23.5271-49.4752$ & 33.0215 & $31.7755-34.2674$ & 161.8051 & 0.9959 \\
\hline
\end{tabular}

Sin embargo, se observó que cuando se utilizan observaciones de temperatura en estado estacionario, cualquier valor por encima de la unidad para el coeficiente de transporte de calor interfacial, $h_{g}$, es útil para describir los datos experimentales, por lo tanto, en la estimación de este parámetro no se logró tener confianza estadística y fenomenológica. Lo 
anterior se puede deber a que el parámetro $h_{\mathrm{g}}$ no juega un papel importante en el gradiente de temperaturas en estado estacionario en el lecho, ya que se tiene aparentemente un pseudo equilibrio térmico local: no obstante, en la descripción de perfiles de temperatura en la transitoriedad, este parámetro puede ser esencial para capturar el comportamiento del sistema. Mencionado lo anterior, para obtener el valor de $h_{g}$ que se utiliza en el modelo pseudo-heterogéneo, se utilizaron valores de $h_{\mathrm{g}}$ de la correlación propuesta por Wakao ${ }^{62}$, debido a que fue obtenida de la transferencia de calor interfacial de una partícula esférica y un fluido a condiciones de $\mathrm{Re}_{\mathrm{p}}$ similares a las obtenidas en este trabajo, ver Tabla 4.2.

\subsubsection{Parámetro térmicos efectivos axiales en estado transitorio}

En este punto, considerando los resultados anteriores, se caracteriza la conductividad térmica efectiva axial ( $\mathrm{k}_{\text {eff,z }}$ ) utilizando un modelo pseudo-homogéneo, y uno pseudo-heterogéneo en estado transitorio, y utilizando los datos experimentales del caso 2, donde se lleva a cabo un experimento de pulsos de flujo de fluido, y se registra el estado transiente en una posición axial del lecho quasi-adiabático. Esto se realiza con el objetivo de analizar la descripción de la transitoriedad cuando se considera un pseudo equilibrio térmico local entre la fase sólida

y la fase fluida a lo largo de esta, utilizando un modelo pseudo-homogéneo, y evaluando la contribución que pueda tener la fase sólida para la descripción de la transitoriedad al no considerar un pseudo equilibrio térmico local, utilizando un modelo pseudo-heterogéneo.

\section{Modelo pseudo-homogéneo}

En la Figura 4.4 se muestra el perfil dinámico de la temperatura como función del tiempo que se ajustó con el modelo pseudo-homogéneo en estado transitorio (Ec. 3.3.2) y los datos de temperatura obtenidos del experimento de pulsos de flujos de fluido en la posición $\mathrm{z}=0$ cm; mientras que en la Tabla 4.3 se presentan las $k_{\text {eff,z }}$ estimadas de los datos experimentales dinámicos. También se presenta un perfil dinámico para las observaciones experimentales a $\mathrm{z}=5 \mathrm{~cm}$, sin embargo, los puntos de colocación más cercanos estuvieron a $3.24 \mathrm{~cm}$ y 7.41 $\mathrm{cm}$. Ante esto, se muestran las predicciones a estas posiciones axiales y un valor promedio entre ambas, con el fin de señalar que se tiene la misma tendencia que los datos experimentales, a pesar de que un valor promedio sea incorrecto debido a que no existe una linealidad entre los puntos de colocación y los perfiles dinámicos de las diferentes posiciones 
axiales. Aunado a lo anterior, un adecuado punto de colocación en la posición $\mathrm{z}=5 \mathrm{~cm}$, podría describir de manera similar, el perfil obtenido en la posición $\mathrm{z}=0 \mathrm{~cm}$.

Se observa que el modelo, que describió los perfiles de temperatura en estado estacionario, ver Figura 4.1, no describió la transitoriedad del perfil de temperatura; particularmente el modelo predice que el estado estacionario se obtiene entre un 500\% y 700\% más rápido que el tiempo observado en el experimento dinámico. A pesar de que el modelo describe ligeramente el error experimental de $\pm 2.5 \mathrm{~K}$, mostrado en una región sombreada alrededor del dato experimental en la Figura 4.4, los datos estadísticos de la estimación de los parámetros efectivos muestran un $\mathrm{R}^{2}$ entre 0.84 hasta 0.96 en la mayoría de los casos, lo cual significa, estadísticamente, una deficiente descripción de los datos experimentales por parte del modelo. Además, los intervalos de confianza del $95 \%$ no son mayores al $\pm 1 \%$ del parámetro efectivo, lo cual representa una alta sensibilidad a las condiciones de operación a las que se están estimando.

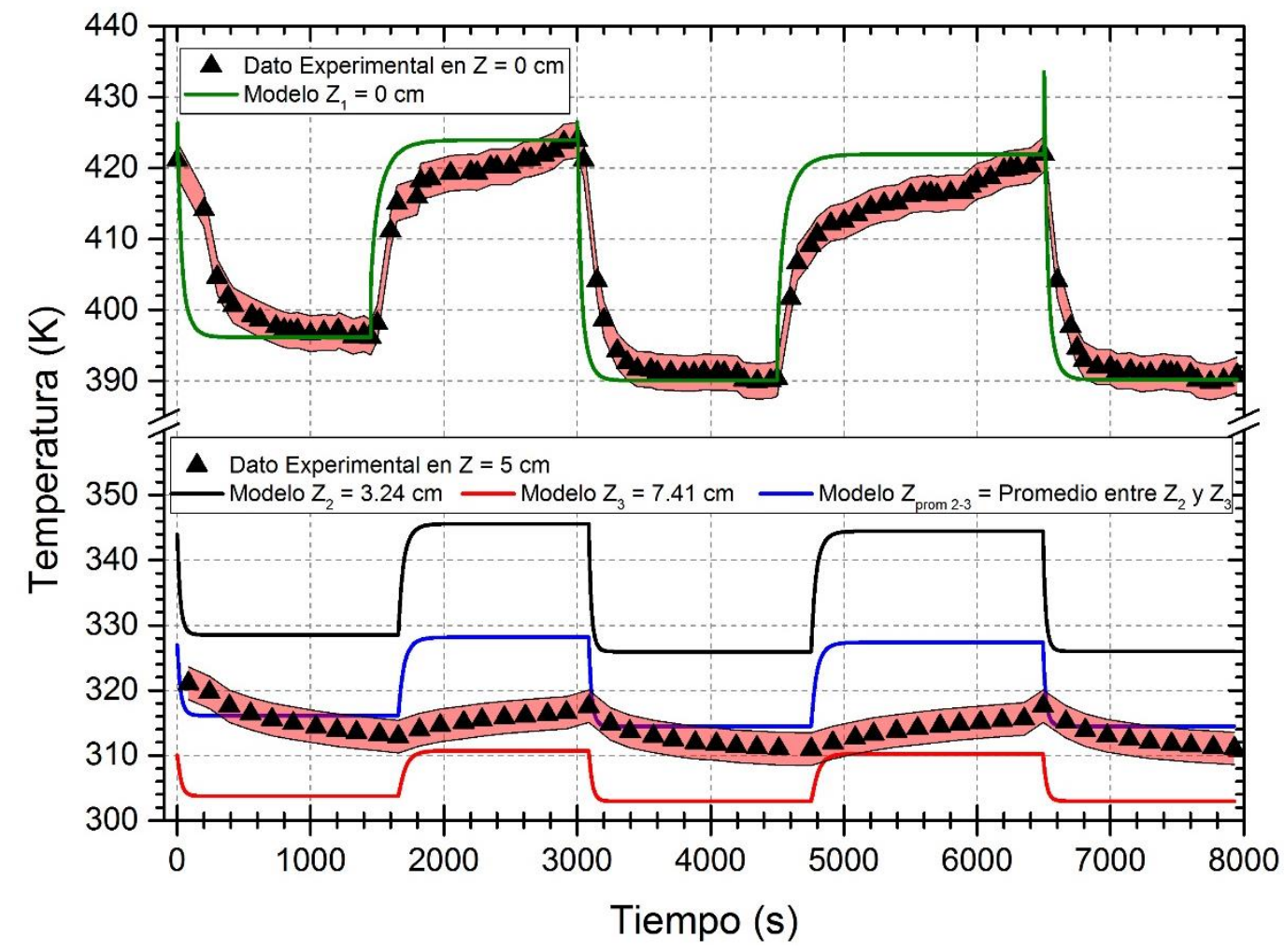

Figura 4.4. Perfil dinámico de temperatura en $\mathrm{z}=0 \mathrm{~cm}$ y $5 \mathrm{~cm}$ en el lecho quasi-adiabático, modelo pseudo-homogéneo $(-)$, dato experimental ( $\boldsymbol{\Delta}$ ), la región sombreada representan los $\pm 2.5 \mathrm{~K}$ de error experimental. 


\begin{tabular}{|c|c|c|c|}
\hline \multicolumn{4}{|c|}{ Tabla 4.3. Keff, $z$ de experimento dinámico, modelo } \\
pseudo-homogéneo \\
\hline $\begin{array}{c}\text { Flujo de } \\
\text { aire (I/min) }\end{array}$ & keff, (W/m K) & I.C. 95\% & $\mathbf{R}^{2}$ \\
\hline 15.4 & 17.9757 & $17.9744-17.9770$ & 0.9053 \\
\hline 8.65 & 11.9992 & $11.9987-11.9997$ & 0.9046 \\
\hline 16.55 & 18.9912 & $18.9881-18.9943$ & 0.9196 \\
\hline 8.86 & 12.0793 & $12.0781-12.0804$ & 0.8459 \\
\hline 16.50 & 18.4442 & $18.4432-18.4452$ & 0.9599 \\
\hline
\end{tabular}

Se considera que el modelo no describió la transitoriedad de la temperatura porque la contribución del sólido no se ha evaluado correctamente, esto se puede deber a que el modelo intenta capturar la información de todos los mecanismos de transferencia de calor que suceden en el lecho con un solo parámetro efectivo, keff,z. De esta forma, el modelo pseudoheterogéneo, al considerar tres parámetros de transferencia de calor, pero esencialmente $h_{g}$, podría describir los datos observados de temperatura del experimento dinámico.

\section{Modelo pseudo-heterogéneo}

El modelado en estado estacionario permitió corroborar la aproximación del pseudo equilibrio térmico local, es decir, considerar que ambas fases, la fluida y la sólida, tienen una diferencia de temperaturas despreciable al estado estacionario. ${ }^{5,8,9,23,28,38,40,50,51}$ En este sentido, se considera que en el estado transitorio, esto no debería ser cierto por la capacidad térmica específica de cada fase, por lo que se utiliza un modelo pseudo-heterogéneo para describir la temperatura de ambas fases cuando se opera el lecho en estado dinámico.

En la Figura 4.5 se presentan los resultados obtenidos con el modelo pseudo-heterogéneo en estado transitorio para describir el experimento dinámico del lecho quasi-adiabático en $\mathrm{z}=0$ cm; mientras que en Tabla 4.4 se muestran los parámetros térmicos efectivos estimados de los datos experimentales dinámicos. Al igual que en el caso pseudo-homogéneo, también se presentan los perfiles para $3.24 \mathrm{~cm}, 7.41 \mathrm{~cm}, \mathrm{y}$ su promedio, junto con los datos experimentales de $\mathrm{z}=5 \mathrm{~cm}$. 


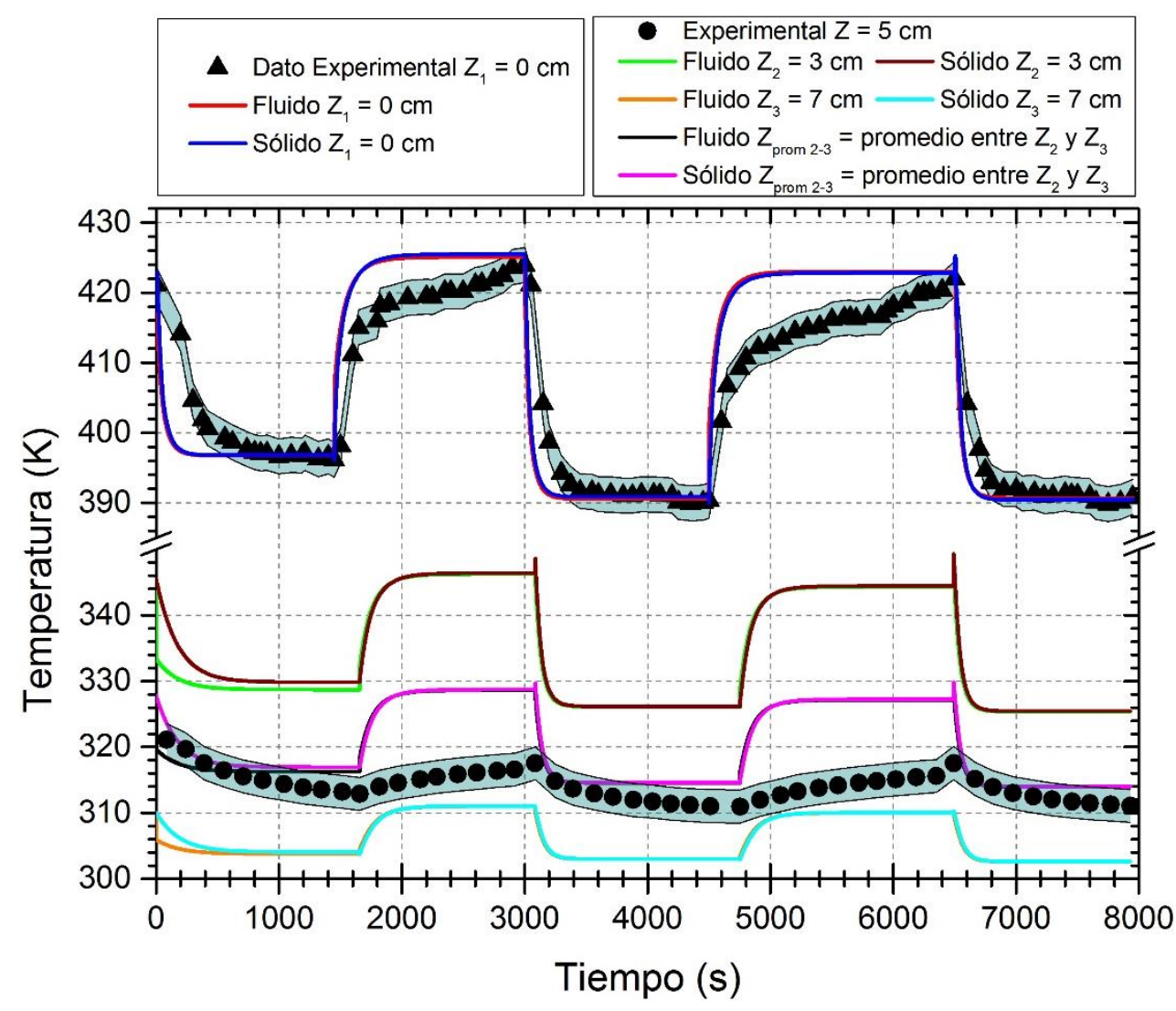

Figura 4.5. Perfil dinámico de temperatura en $\mathrm{z}=0 \mathrm{~cm}$ y $5 \mathrm{~cm}$ en el lecho quasi-adiabático, modelo pseudo-heterogéneo (一), la región sombreada representan los $\pm 2.5 \mathrm{~K}$ de error experimental.

\begin{tabular}{|c|c|c|c|c|c|c|c|}
\hline \multicolumn{8}{|c|}{$\begin{array}{l}\text { Tabla 4.4. Parámetros térmicos efectivos del experimento dinámico, modelo pseudo- } \\
\text { heterogéneo }\end{array}$} \\
\hline $\begin{array}{c}\text { Flujo } \\
\text { de aire } \\
\text { (1/min) }\end{array}$ & $\begin{array}{c}\mathbf{k s , e f f , z} \\
(\mathbf{W} / \mathbf{m ~ K})\end{array}$ & $\begin{array}{c}\text { I.C. } \\
95 \% \\
k_{\text {s,eff,z }}\end{array}$ & $\begin{array}{c}\mathbf{k f , e f f , z} \\
(\mathbf{W} / \mathbf{m} \mathbf{K})\end{array}$ & $\begin{array}{c}\text { I.C. } \\
95 \% \\
\mathbf{k}_{f, \text { eff,z }}\end{array}$ & $\begin{array}{c}\mathbf{h}_{\mathbf{g}} \\
\left(\mathbf{W} / \mathbf{m}^{2} \mathbf{K}\right)\end{array}$ & $\begin{array}{l}\text { I.C. } \\
95 \% \\
k_{s, e f f, z}\end{array}$ & $\mathbf{R}^{2}$ \\
\hline 15.4 & 18.0386 & $\begin{array}{l}16.2535 \\
19.8237\end{array}$ & 17.9721 & $\begin{array}{l}17.9782 \\
17.9861\end{array}$ & 60.7442 & $\begin{array}{l}51.2716 \\
70.2169\end{array}$ & 0.9208 \\
\hline 8.65 & 12.0132 & $\begin{array}{l}11.0662 \\
12.9603\end{array}$ & 12.0022 & $\begin{array}{l}11.9998 \\
12.0045\end{array}$ & 42.3885 & $\begin{array}{l}34.2242 \\
50.5528\end{array}$ & 0.8835 \\
\hline 16.55 & 20.7276 & $\begin{array}{l}18.4595 \\
22.9957\end{array}$ & 18.9777 & $\begin{array}{l}18.9564 \\
18.9990\end{array}$ & 75.5766 & $\begin{array}{l}72.8833 \\
77.1168\end{array}$ & 0.9297 \\
\hline 8.86 & 13.3980 & $\begin{array}{l}12.9220 \\
13.8740\end{array}$ & 12.0039 & $\begin{array}{l}11.9994 \\
12.0084\end{array}$ & 45.6149 & $\begin{array}{l}39.4156 \\
51.8142\end{array}$ & 0.8162 \\
\hline 16.50 & 18.9157 & $\begin{array}{l}14.7329 \\
23.0986\end{array}$ & 18.4956 & $\begin{array}{l}18.9816 \\
19.0096\end{array}$ & 70.0176 & $\begin{array}{l}55.4399 \\
84.5953\end{array}$ & 0.9688 \\
\hline
\end{tabular}


En la descripción del modelo pseudo-heterogéneo no hay diferencia de temperaturas apreciable entre la temperatura de la fase fluida y la fase sólida, asimismo, tampoco hay una diferencia apreciable a lo obtenido con el modelo pseudo-homogéneo. Es decir, no se logra describir adecuadamente la transitoriedad de la temperatura observada en el lecho operado de forma adiabática; las conductividades, tanto para el sólido como para el fluido, son iguales desde un punto de vista estadístico, inclusive se puede observar que las conductividades térmicas efectivas estimadas para cada fase no son muy diferentes de las estimadas en el modelo pseudo-homogéneo, ver Tabla 4.3, debido a que los valores de $\mathrm{R}^{2}$ permanecen casi iguales, mientras que los I.C. del 95\% siguen mostrando una alta sensibilidad para la fase fluida, aunque para la fase sólida existe un $\pm 10 \%$ en estos intervalos con respecto a la $\mathrm{k}_{\mathrm{s}, \mathrm{eff}, \mathrm{z}}$ estimada. Sin embargo, la estimación del $h_{g}$ muestra valores menores a los obtenidos por la correlación utilizada en el modelo en estado estacionario.

Aunado a lo anterior, no se tiene confianza estadística y fenomenológica en la determinación del coeficiente de transferencia de calor interfacial, $h_{\mathrm{g}}$. La no adecuada descripción de la transitoriedad se puede atribuir a que el modelo no captura adecuadamente la información de la capacidad térmica específica empleada en el término transitorio del modelo y/o de la transferencia de calor que captura los fenómenos en la capa límite mediante el uso del coeficiente de transferencia de calor interfacial $h_{\mathrm{g}}$. Un estudio más detallado sobre la capacidad térmica efectiva y la estimación de $h_{\mathrm{g}}$ se presenta en la siguiente sección.

\subsubsection{Transferencia de calor interfacial y capacidad térmica específica}

Como se mencionó anteriormente, a diferencia del modelo pseudo-homogéneo, el modelo pseudo-heterogéneo considera tres parámetros efectivos que caracterizan los mecanismos de transferencia de calor que ocurren dentro del lecho empacado; también se mencionó que las conductividades efectivas, tanto de la fase sólida como de la fase fluida, no afectan de manera significativa la transitoriedad en el lecho sino solamente el gradiente de temperaturas que se obtiene en estado estacionario; es por esto que en las Figuras 4.6, 4.7 y 4.8 se presenta un estudio de la sensibilidad del parámetro $h_{\mathrm{g}}$ a las condiciones de operación del experimento dinámico cuando se aumenta el flujo de aire de 9 a 15.4 1/min.

En estas figuras se observa que la magnitud de $h_{g}$ influye en la transitoriedad de la temperatura, esencialmente la de la fase sólida. Un valor entre 1 y 5 de $\mathrm{h}_{\mathrm{g}}$ predice un perfil 
de temperatura de la fase sólida similar a las tendencias experimentales. No obstante, para la fase fluida, las predicciones son similares a las que se observaron en las secciones anteriores, es decir el estado estacionario se alcanza menos de 5 segundos. El perfil de la temperatura promedio entre ambas fases como función del tiempo permite observar que la fase sólida juega un papel esencial en la descripción de experimentos transientes de transferencia de calor, debido al gradiente que existe entre ambas fases en la transitoriedad.

El transporte de calor interfacial, cuantificado por hg, es un mecanismo que caracteriza la cantidad de calor transferido de una fase a otra, en este caso del sólido al fluido, por lo que es crucial la estimación adecuada del parámetro $h_{g}$ en condiciones transientes para caracterizar el tiempo en el que ambas fases llegan a un equilibrio térmico, en el estado estacionario. En este sentido, es importante comentar que el valor de $h_{g}$ que describe las tendencias experimentales no tiene un orden de magnitud similar al que se reporta en la literatura.

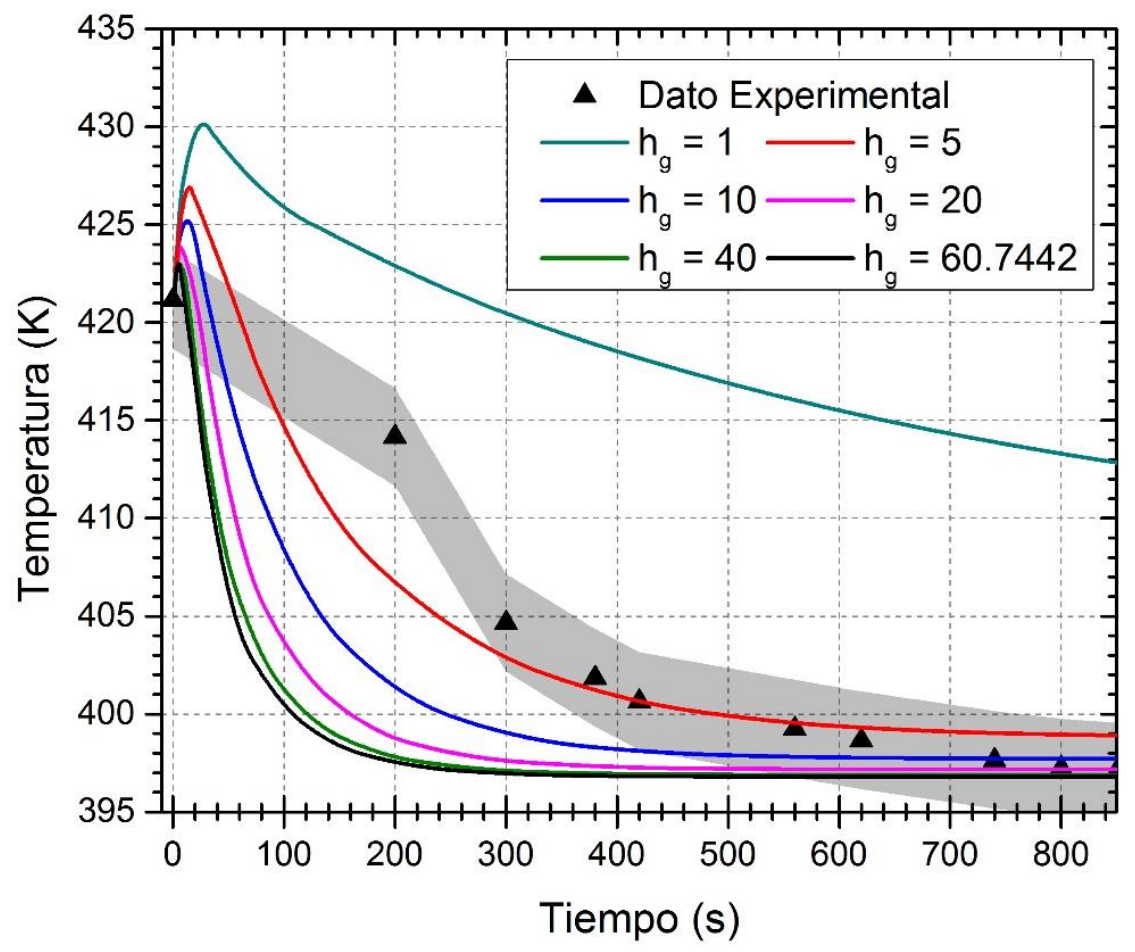

Figura 4.6. Perfil dinámico de temperatura a $\operatorname{Re}_{\mathrm{p}}=288.60$ con diferentes $\mathrm{h}_{\mathrm{g}}$ en $\mathrm{z}=0 \mathrm{~cm}$ en el lecho quasi-adiabático, modelo pseudo-heterogéneo, fase sólida. 


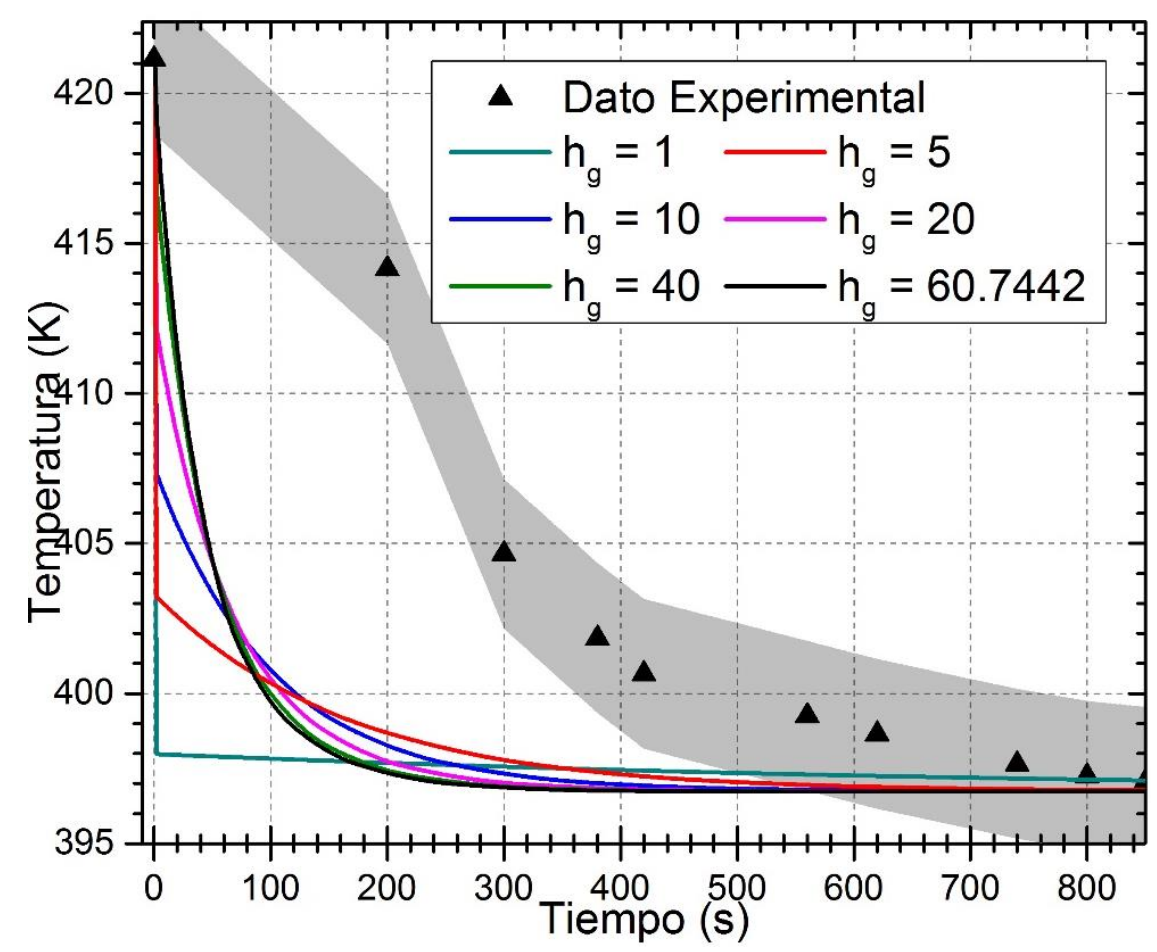

Figura 4.7. Perfil dinámico de temperatura a $R_{p}=288.60$ con diferentes $h_{g}$ en $z=0 \mathrm{~cm}$ en el lecho quasi-adiabático, modelo pseudo-heterogéneo, fase fluida.

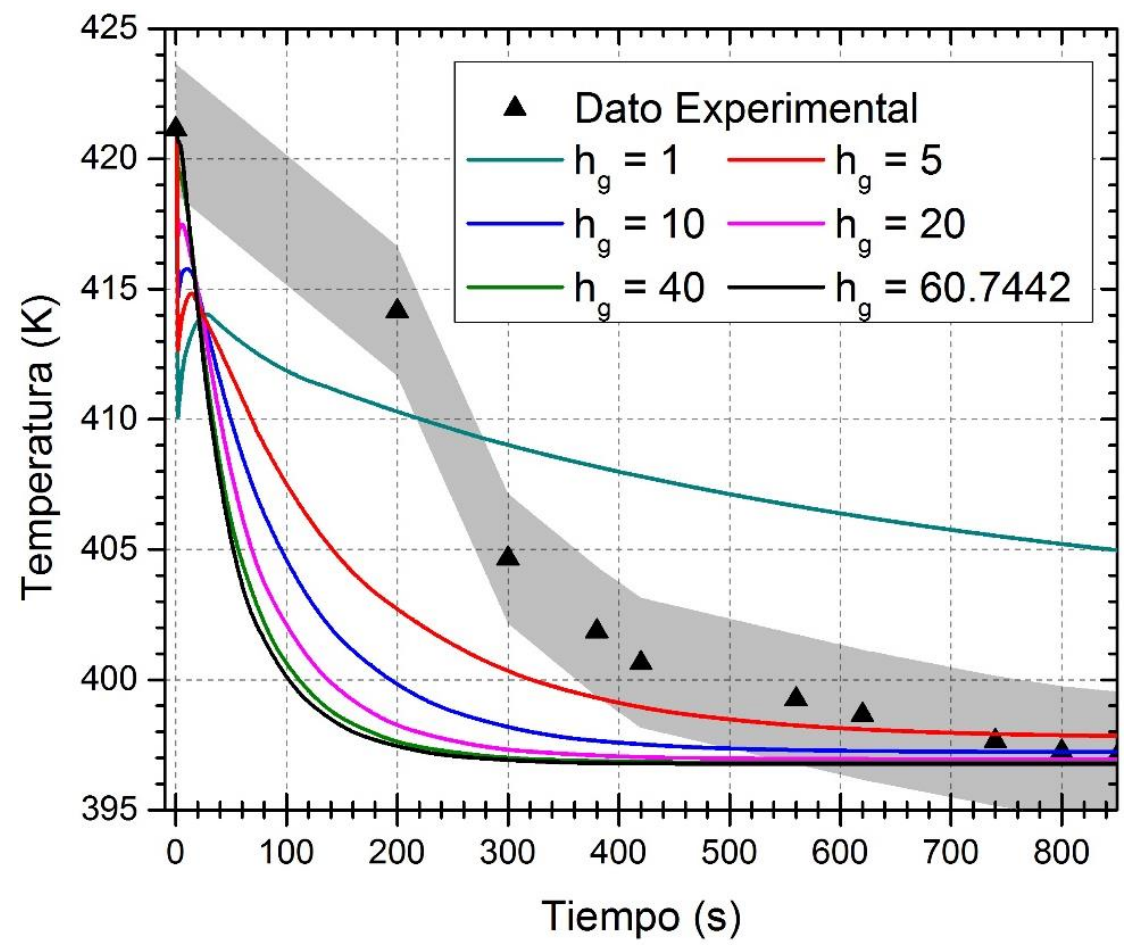

Figura 4.8. Perfil dinámico de temperatura a $\mathrm{Re}_{\mathrm{p}}=288.60$ con diferentes $\mathrm{h}_{\mathrm{g}}$ en $\mathrm{z}=0 \mathrm{~cm}$ en el lecho quasi-adiabático, temperatura promedio entre ambas fases. 
Si bien la transferencia de calor interfacial aparenta influir en la transitoriedad de los experimentos de transferencia de calor dinámicos, aún se sigue prediciendo un estado estacionario a un tiempo menor que el observado experimentalmente, aunado al hecho de que se tiene incertidumbre en la magnitud de $h_{\mathrm{g}}$ el parámetro que caracteriza este mecanismo. Esto nos lleva a considerar que la capacidad térmica volumétrica de la fase sólida también podría jugar un papel importante en la descripción de la transitoriedad en los perfiles de temperatura.

Debido a que las propiedades termofísicas del sólido (e.g. $\mathrm{Cp}_{\mathrm{s}}$ ) fueron obtenidas para una sola partícula ${ }^{18}$, y el modelo describe los mecanismos de transferencia en una pseudo fase sólida, es decir, un esqueleto compuesto por todas las partículas dentro del lecho, el cuál puede ser considerado como un sólido con alta porosidad por la cual fluye la fase fluida, se puede hipotetizar que la cantidad de calor necesaria para elevar un gramo del esqueleto un grado centígrado, el calor específico del sólido, $\mathrm{Cp}_{\mathrm{s}}$, debería ser diferente al obtenido para una sola partícula no porosa. El efecto que tiene la capacidad térmica volumétrica del sólido se presenta en las Figura 4.9 y 4.10, en donde se muestran los perfiles de temperatura en las condiciones de operación del experimento dinámico cuando se aumenta el flujo de aire de 9 a 15.4 1/min, como se hizo en las Figuras 4.6, 4.7 y 4.8, pero ahora variando los valores de $\mathrm{Cp}_{\mathrm{s}}$. Cabe señalar que los valores de las conductividades térmicas efectivas de ambas fases y el coeficiente de transferencia de calor interfacial que se usan en estas simulaciones son los valores estimados del modelo pseudo-heterogéneo, ver Tabla 4.4.

Los resultados obtenidos al variar el $\mathrm{Cp}_{\mathrm{s}}$ muestran que un valor entre 4 y 5 veces mayor al reportado para el sólido que se utiliza en este trabajo ${ }^{5,18}$, el calculado para una sola partícula sólida, predice adecuadamente el perfil transitorio de temperatura en el lecho. Esto sugiere que el esqueleto conformado por las partículas requieren de, aproximadamente, entre 4 y 5 veces más calor para elevar un gramo del esqueleto un grado centígrado, es decir, al requerir más energía, retardan el tiempo en el que se alcanza el estado transitorio de la transferencia de energía en el lecho, tal y como las observaciones experimentales lo mostraron. Lo anterior sugiere una estimación del $\mathrm{Cp}_{\mathrm{s}}$ del esqueleto contenido en el lecho, lo cual implica que cada lecho debe de tener su propio $\mathrm{Cp}_{\mathrm{s}}$, al igual que los parámetros de transferencia de calor, un resultado que hasta donde se tiene conocimiento no se ha reportado en la literatura. 


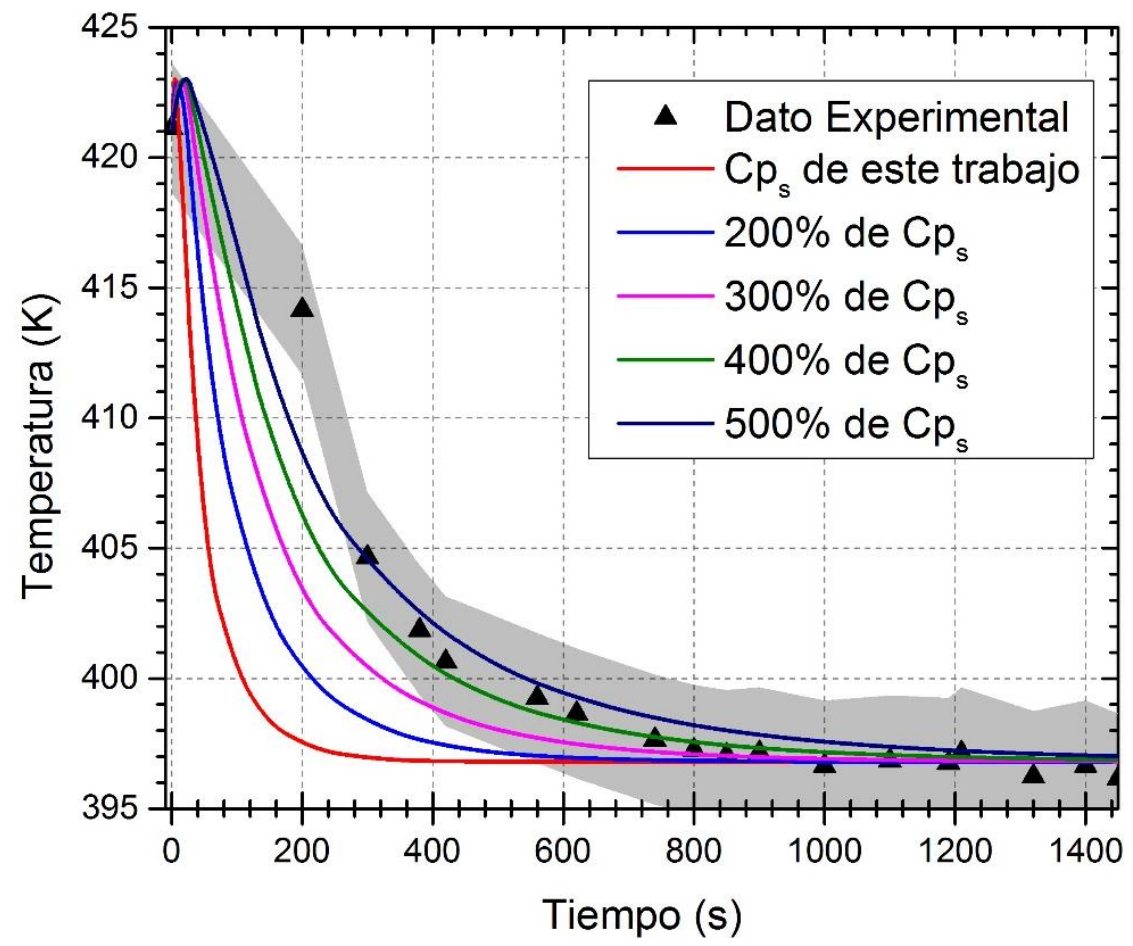

Figura 4.9. Perfil dinámico de temperatura a $\mathrm{Re}_{\mathrm{p}}=288.60$ con diferentes $\mathrm{Cp}_{\mathrm{s}}$, en $\mathrm{z}=0 \mathrm{~cm}$ en el lecho quasi-adiabático, fase sólida.

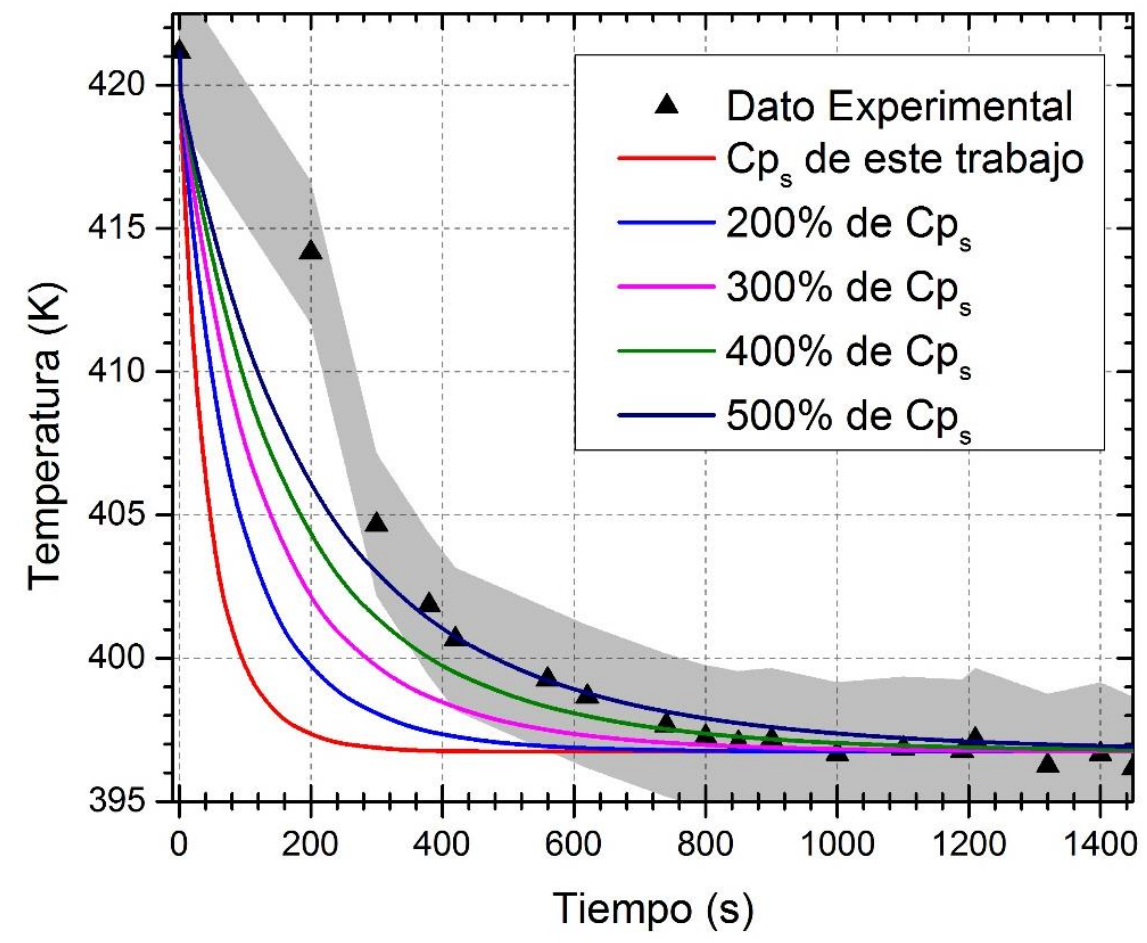

Figura 4.10. Perfil dinámico de temperatura a $\operatorname{Re}_{\mathrm{p}}=288.60$ con diferentes $C \mathrm{p}_{\mathrm{s}}$, en $\mathrm{z}=0 \mathrm{~cm}$ en el lecho quasi-adiabático, fase fluida. 


\subsection{Lecho no adiabático no isotérmico}

En esta sección se presentan los resultados de transferencia de calor que se obtuvieron para el caso experimental 3, el lecho con calentamiento en la pared, considerando el $\operatorname{Re}_{\mathrm{p}}$ de 163.36 para las diferentes aproximaciones. Posteriormente, se escoge entre las aproximaciones aquella que represente adecuadamente los mecanismos de transferencia de calor en el lecho, para emplearla en la caracterización de la transferencia de calor para cuando se tengan $\operatorname{Re}_{\mathrm{p}}$ de 40.83 y 81.67 . Para la caracterización de la transferencia de calor en el lecho empacado con calentamiento en la pared a $\operatorname{Re}_{\mathrm{p}}$ de 163.36 se utiliza el modelo pseudo-homogéneo que describe el comportamiento de un lecho que opera de forma no isotérmica y no adiabática, Ec. 3.3.4. Este modelo, además de hacer uso de la keff,z que se determinó del estudio de transferencia de calor axial, se utiliza para estimar los parámetros de transferencia de calor que caracterizan los mecanismos correspondientes en la dirección radial del lecho. Estos parámetros se estiman mediante el ajuste de los perfiles de temperatura observados en direcciones axial y radial. Con el objetivo de tener mayor confianza estadística en la determinación de los parámetros de transferencia de calor radial, se extendió la base de datos experimentales observados mediante una interpolación, lo que permitió obtener veintidós datos axiales con veintidós datos radiales de temperatura en lugar de diez y cinco temperaturas, respectivamente. Para evaluar el modelo se utilizarán varias aproximaciones con el objetivo de realizar una evaluación de las diferencias entre estas. Cabe señalar que el área sombreada alrededor de los datos experimentales, en las figuras, representa el $\pm 2.5 \mathrm{~K}$ de error experimental.

\subsubsection{Aproximación clásica}

Esta aproximación considera un flujo pistón, una velocidad intersticial constante dentro del lecho, y la condición de frontera de convección superficial en la pared (Ec. 3.3.4 a-f), utilizando un coeficiente de transferencia de calor de pared, $\mathrm{h}_{\mathrm{w}}$.

En las Figuras 4.11 y 4.12 se presentan los ajustes de los perfiles de temperaturas axial y radial, respectivamente, obtenidos con el modelo (Ec. 3.3.4 a-f) a $\operatorname{Re}_{\mathrm{p}}=163.36$. Se observa que desde la entrada del lecho hasta los $30 \mathrm{~cm}$, aproximadamente, se tiene un buen ajuste de los perfiles de temperatura, sin embargo, a la salida del lecho, los últimos $10 \mathrm{~cm}$, el modelo predice una temperatura menor que la observada en la región central del lecho. 


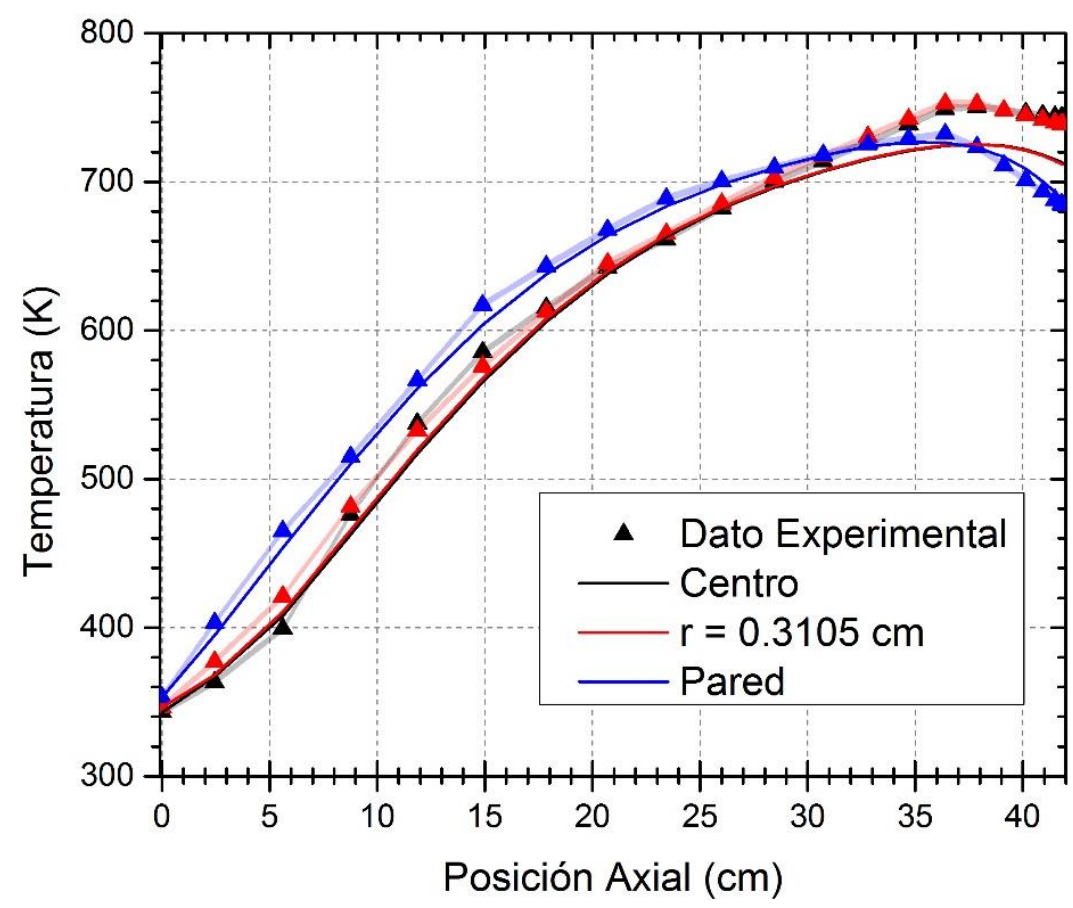

Figura 4.11. Perfil axial de temperatura en el lecho no isotérmico no adiabático utilizando la aproximación clásica con un $\operatorname{Re}_{\mathrm{p}}=163.36$ a 3 posiciones radiales diferentes;

(ム) dato experimental, (-) modelo.

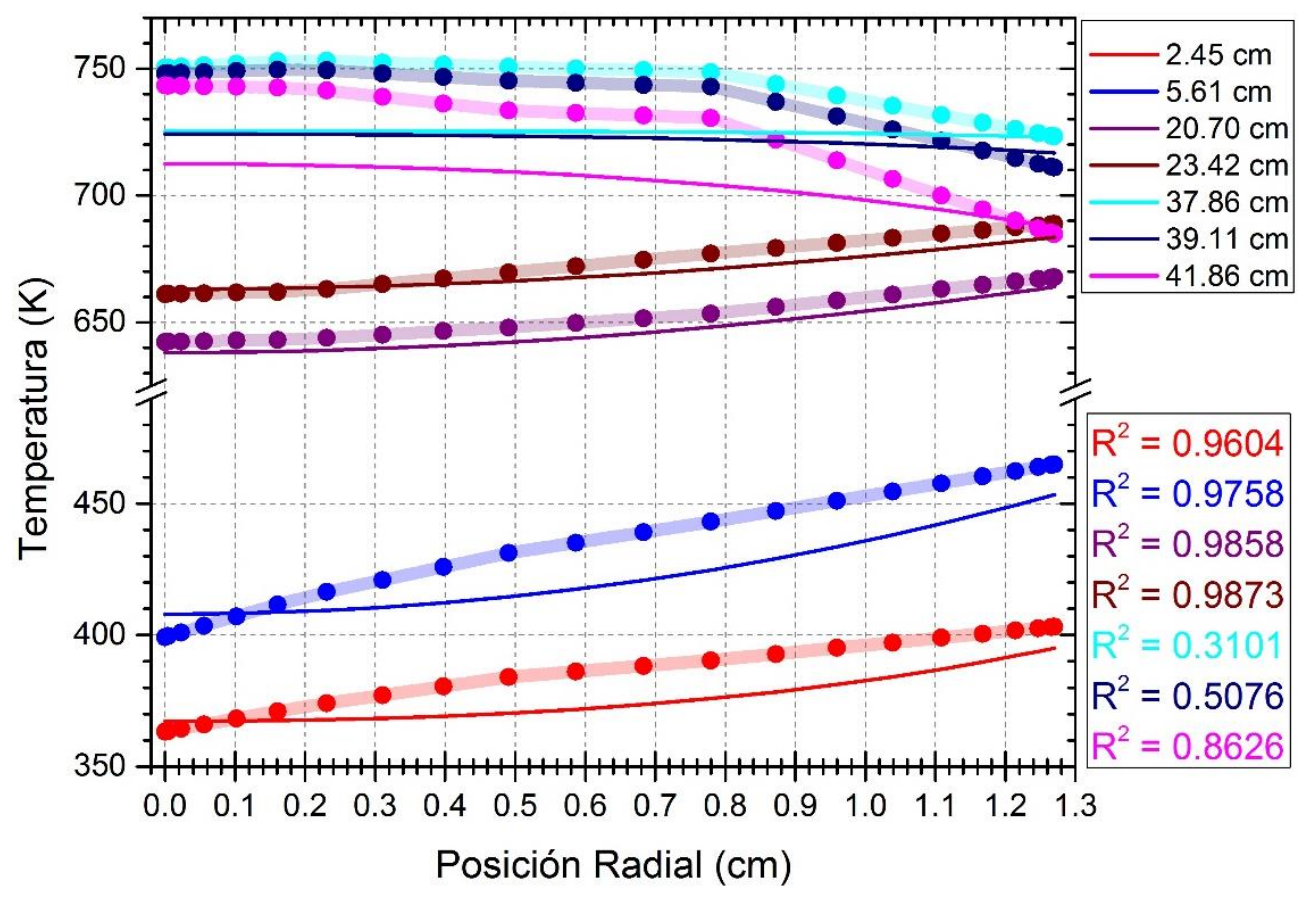

Figura 4.12. Perfil radial de temperatura en el lecho no isotérmico no adiabático utilizando la aproximación clásica con un $\operatorname{Re}_{\mathrm{p}}=163.36$ a 7 posiciones axiales diferentes;

$(\bullet)$ dato experimental, (-) modelo. 
El coeficiente de correlación $\mathrm{R}^{2}$ en el perfil axial de temperaturas ubicadas en el centro del lecho es de 0.9986, mientras que el ajuste en la pared tiene un $\mathrm{R}^{2}$ de 0.9997 , esto demuestra que estadísticamente se tiene una aproximación adecuada de los perfiles axiales de temperatura predichos. Un acercamiento respecto a la mala predicción a la salida del lecho se puede ver en la Figura 4.12 , en donde a $41.86 \mathrm{~cm}, 39.11 \mathrm{~cm}$ y $37.86 \mathrm{~cm}$, existe un $\mathrm{R}^{2} \mathrm{de}$ 0.8626, 0.5076 y 0.3101 , respectivamente, entre los datos observados y el perfil de temperatura radial predicho. Los valores estimado de $k_{\text {eff, }} \mathrm{y} \mathrm{h}_{\mathrm{w}}$ para este caso son 0.5627 $\mathrm{W} / \mathrm{m} \mathrm{K}$ y $446.54 \mathrm{~W} / \mathrm{m}^{2} \mathrm{~K}$, respectivamente, donde los I.C. del $95 \%$ fueron de 0.5626 0.5628 , para la $k_{\text {eff,r, }}$, y $99.6764-697.73$ para $h_{w}$. Cabe señalar que el valor estimado $h_{w}$ es diferente a los obtenidos con las correlaciones de la literatura presentadas en la Figura 2.3, en donde se tienen diferencias entre el $48 \%$ y el $97 \%$ con respecto al $h_{w}$ estimado. Por otro lado, los valores de $\mathrm{k}_{\text {eff,r }}$ obtenidos con correlaciones reportadas en la literatura, ver la Figura 2.4, presentan una diferencia entre el $21 \%$ y $25 \%$ con respecto a la keff,r estimada, y su intervalo de confianza. Para la estimación de los parámetros efectivos se emplearon todas las temperaturas axiales y radiales observadas del lecho. La no adecuada predicción de la temperatura a la salida en el lecho, se puede deber a una pérdida de calor a la salida del lecho. Sin embargo, el sistema experimental estuvo aislado en esa zona para evitar las pérdidas de calor, lo cual hace suponer que la condición de frontera utilizada es adecuada. También cabe señalar que la no adecuada consideración de la hidrodinámica afectan de manera significativa la predicción de los perfiles de temperatura en el lecho, y que los parámetros efectivos de transferencia de calor no son capaces de capturar los mecanismos de transferencia de calor que ocurren como un posible efecto de los gradientes de temperatura que se tienen en cada región del lecho.

\subsubsection{Aproximación con hidrodinámica}

Esta aproximación utiliza el mismo modelo que la aproximación clásica, pero considera la hidrodinámica de acuerdo a la metodología propuesta por Aparicio et al. ${ }^{3}$ para obtener los perfiles de velocidad axial que afectan el término convectivo de la ecuación de transferencia de calor. Con esta aproximación se evalúan tanto el caso cuando se considera un coeficiente de transferencia de calor de pared, $\mathrm{h}_{\mathrm{w}}$, en la condición de frontera (ec.3.3.4 a-f), y la condición de frontera dada por la Ec. 3.3.4 a-e, g, la aproximación de la capa límite. Para el caso donde 
sólo se utiliza la $\mathrm{k}_{\mathrm{eff}, \mathrm{r}}$ se considera que este parámetro captura todos los mecanismos de transferencia de calor radiales en el interior del lecho, incluyendo la región cercana a la pared $^{5}$, no obstante, para emplear esta aproximación es necesario contar con una medición de temperatura en la pared interna del lecho, la cual para este caso se tiene.

\section{Condición de frontera incluyendo keff,r $\mathbf{y} \mathbf{h}_{\mathbf{w}}$}

Los ajustes del modelo pseudo-homogéneo en estado transitorio considerando la hidrodinámica y en la condición de frontera el uso de $\mathrm{h}_{\mathrm{w}}$ se presentan en las Figura 4.13 y 4.14. La Figura 4.13 presenta los perfiles axiales de temperatura en tres posiciones radiales del lecho, mientras que la Figura 4.14 presenta los perfiles radiales de temperatura que se tienen en siete posiciones axiales del lecho. Cabe señalar que para la estimación de los parámetros efectivos se emplearon todas las temperaturas axiales y radiales observadas en el lecho.

Esta aproximación tampoco puede ajustar las observaciones en los últimos $10 \mathrm{~cm}$ del lecho. El valor estimado de $\mathrm{k}_{\text {eff,r }}$ es de $0.7200 \mathrm{~W} / \mathrm{m} \mathrm{K}$, la cual es un $27 \%$ mayor al determinado con la aproximación clásica. La diferencia de este parámetro con respecto a los valores obtenidos con las correlaciones reportadas en la literatura, ver Figura 2.4, está entre 5\% y 80\%. El valor estimado de $h_{w}$ es de $447.9895 \mathrm{~W} / \mathrm{m}^{2} \mathrm{~K}$, donde a diferencia de lo que se reporta en la literatura, ver Figura 2.3, es similar al que se obtiene con la aproximación que considera flujo pistón, aunque se debe aclarar que los intervalos de confianza para este parámetro son muy amplios. Los intervalos de confianza del $95 \%$ para la $\mathrm{k}_{\mathrm{eff}, \mathrm{r}} \mathrm{y} \mathrm{h}_{\mathrm{w}}$ fueron de $0.7198-0.7201$, y 102.9999 - 698.9948, respectivamente.

El coeficiente de correlación, $\mathrm{R}^{2}$, para el perfil axial de temperaturas ubicado en el centro del lecho es de 0.9985 , y el $\mathrm{R}^{2}$ del perfil axial ubicado en la pared es de 0.9993 , lo cual vuelve a indicar, estadísticamente, una predicción parcialmente adecuada de los perfiles axiales de temperatura, mientras que el $\mathrm{R}^{2}$ de los perfiles radiales a la salida del lecho, $37.86 \mathrm{~cm}, 39.11$ cm y $41.86 \mathrm{~cm}$, es de $0.3758,0.2715$ y 0.8428 , respectivamente. Sigue siendo muy deficiente el modelo para predecir los perfiles de temperatura en la zona central del lecho, principalmente en la salida de este. 


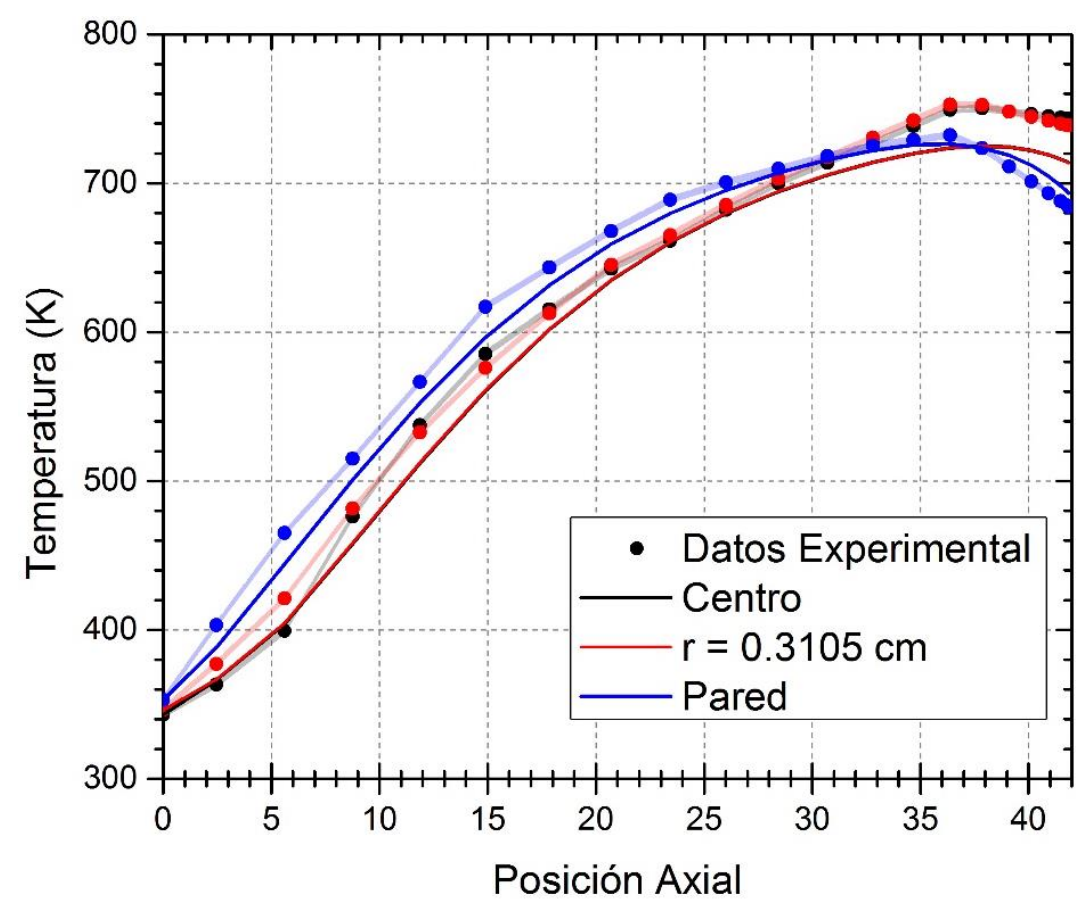

Figura 4.13. Perfil axial de temperatura en el lecho no isotérmico no adiabático con hidrodinámica considerando $h_{w}$ a $\operatorname{Re}_{p}=163.36,3$ posiciones radiales diferentes;

$(\bullet)$ dato experimental, (-) modelo.

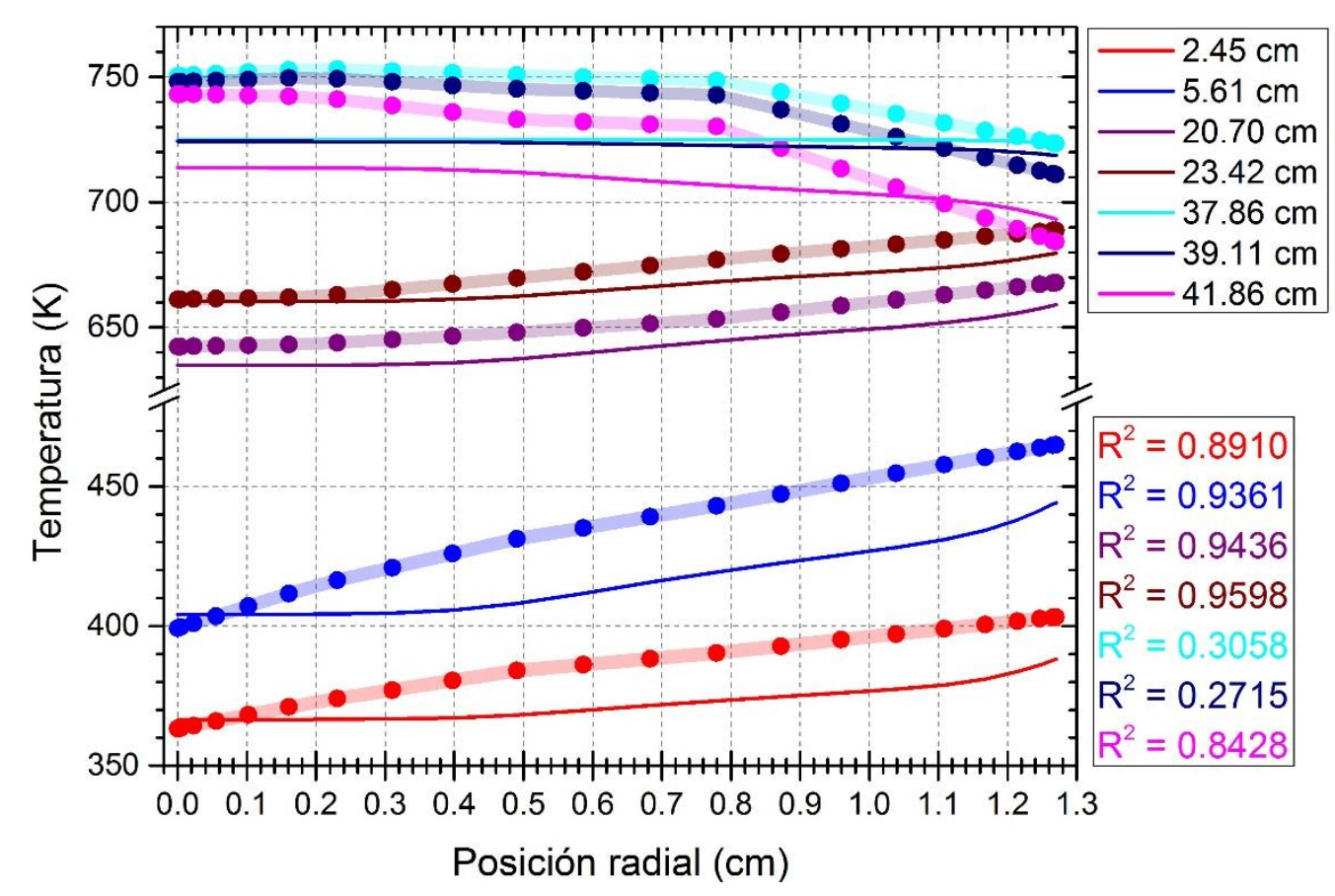

Figura 4.14. Perfil radial de temperatura en el lecho no isotérmico no adiabático con hidrodinámica considerando $h_{\mathrm{w}}$ a $\mathrm{Re}_{\mathrm{p}}=163.36,7$ posiciones axiales diferentes;

(ム) dato experimental, (一) modelo. 
Como se mencionó anteriormente, la no adecuada predicción de la temperatura a la salida del lecho, esencialmente en la región central, se puede deber a una pérdida de calor no capturada experimentalmente y a que la keff,r no es capaz de capturar los mecanismos de transferencia de calor que ocurren como un efecto de los gradientes de temperatura que se tienen, primordialmente en la región central del lecho, debido a que en la región de la pared del lecho el $h_{w}$ aparenta capturar adecuadamente los mecanismos de transferencia de calor.

\section{Aproximación de la capa límite, $\mathbf{T}=\mathbf{T}_{\mathbf{w}}(\mathbf{z})$}

Los perfiles de temperatura axial y radial obtenidos con el modelo de transferencia de calor en estado transitorio con la aproximación de la capa límite se presentan en las Figuras 4.15 y 4.16. Para la estimación se tomaron en cuenta todas las temperaturas axiales y radiales observadas.

El valor estimado de $\mathrm{k}_{\text {eff,r }}$ es de $0.64870 \mathrm{~W} / \mathrm{m} \mathrm{K}$, el cual debe capturar todos los mecanismos de transferencia de calor radial que ocurren dentro del lecho. Este valor presenta una diferencia entre el $4.8 \%$ y $78 \%$ con respecto a los valores obtenidos de las correlaciones reportadas en la literatura, ver Figura 2.4. Los I.C. del 95\% son de 0.64878 - 0.64861, lo que nos indica que este parámetro es estadísticamente confiable. El coeficiente de correlación,

$\mathrm{R}^{2}$, que existe en el perfil axial en el centro del lecho es de 0.9986 , mientras que en la pared es de 0.9999 , mientras que el $\mathrm{R}^{2}$ del perfil radial a las posiciones axiales $\mathrm{z}=41.86 \mathrm{~cm}, 39.11$ cm y $37.86 \mathrm{~cm}$ son $0.8467,0.5642$ y 0.3203 .

Lo anterior demuestra que esta aproximación, al igual que las dos mostradas anteriormente, no captura los mecanismos en todo el lecho empacado, principalmente en la región central y a la salida del lecho. Sin embargo, sus $\mathrm{R}^{2}$ ajustan mejor que las dos aproximaciones mostradas anteriormente. 


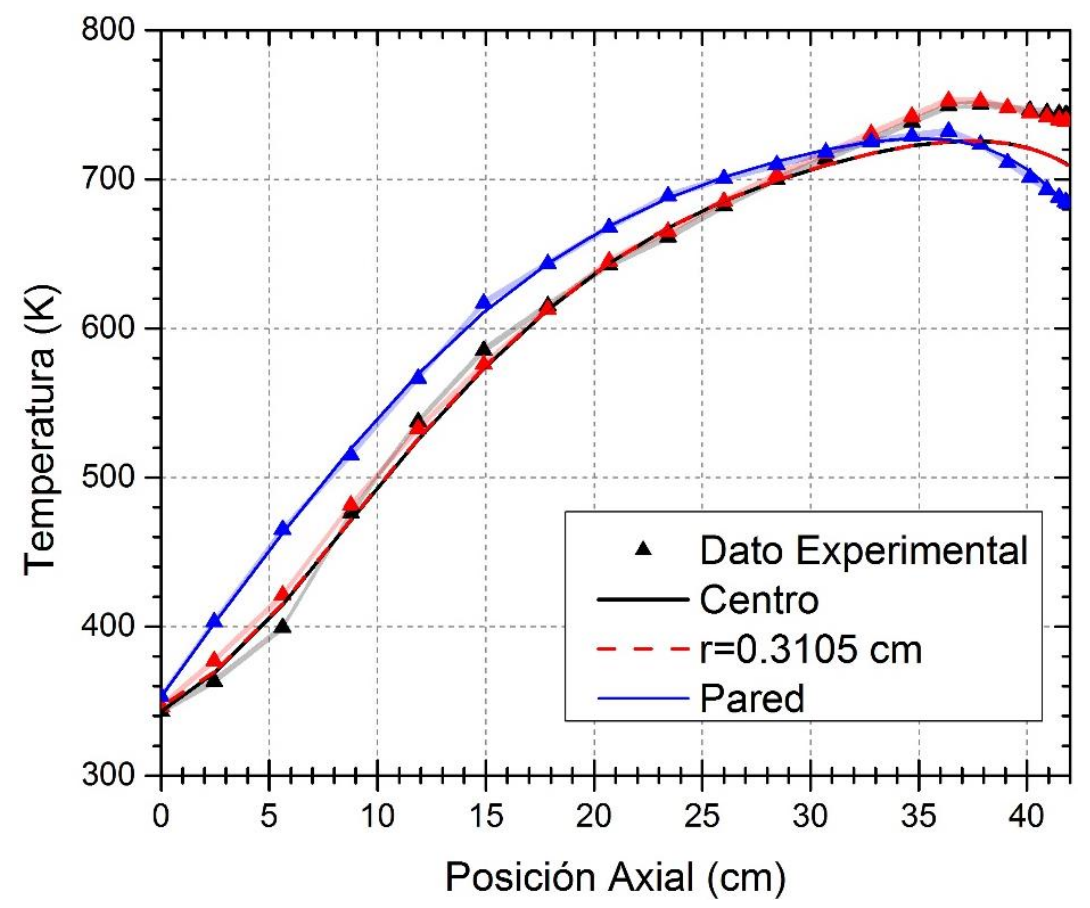

Figura 4.15. Perfil axial de temperatura en el lecho no isotérmico no adiabático con aproximación de la capa límite a $\operatorname{Re}_{\mathrm{p}}=163.36,3$ posiciones radiales diferentes;

(ム) dato experimental, (-) modelo.

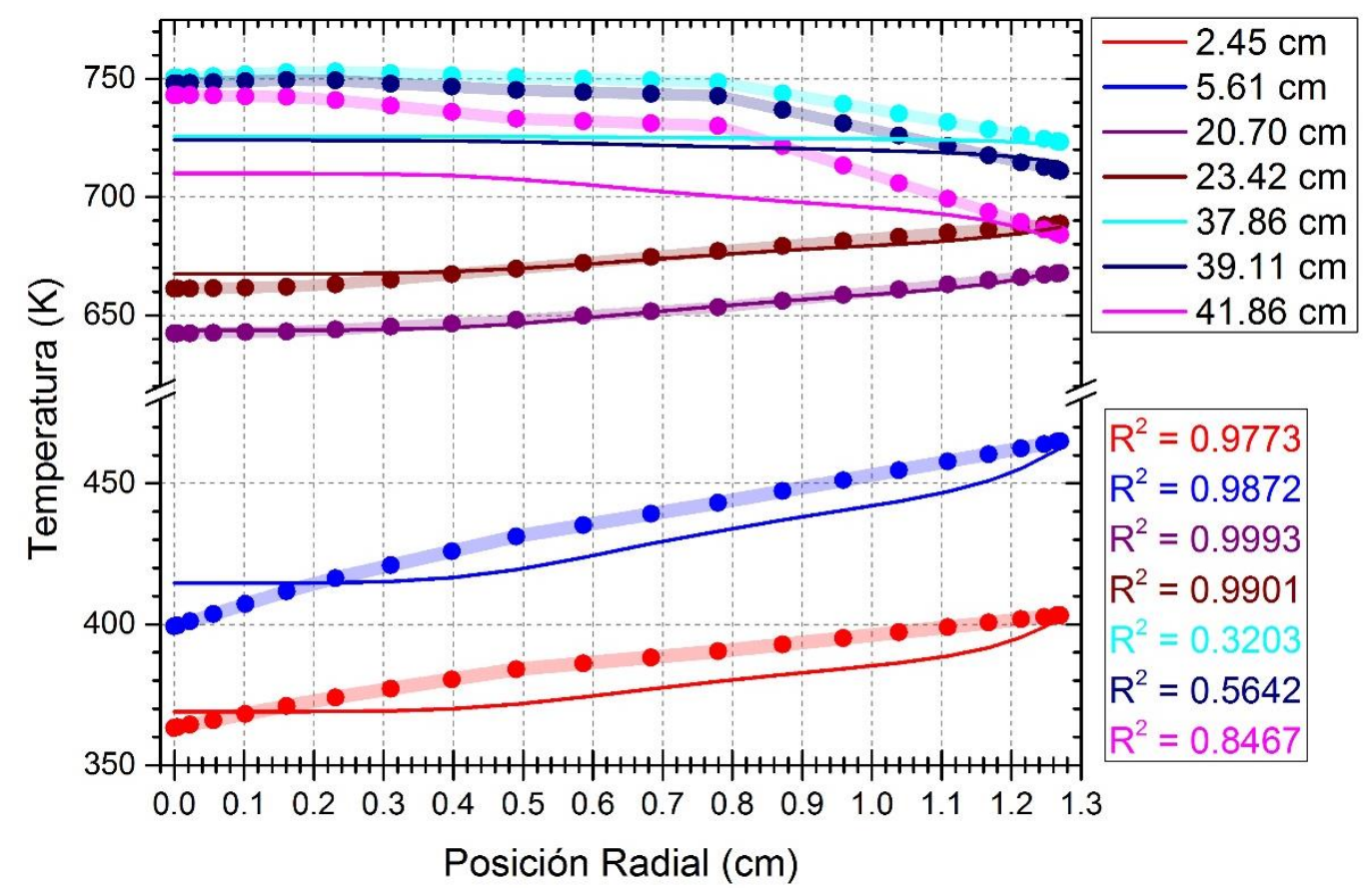

Figura 4.16. Perfil radial de temperatura en el lecho no isotérmico no adiabático con aproximación de la capa límite a $\mathrm{Re}_{\mathrm{p}}=163.36,7$ posiciones axiales diferentes;

(ム) dato experimental, (一) modelo. 
Una posible solución a lo anterior señalado podría ser la inclusión de la componente radial de la velocidad, la cual, como se ha mencionado en estudios de $\mathrm{CFD}^{41,42}$, se debe considerar en el modelado de lechos empacados para capturar adecuadamente los mecanismos de transferencia de calor en la dirección radial.

\subsubsection{Aproximación de capa límite considerando vz y vr}

Hasta donde se tiene conocimiento, en la actualidad no hay estudios experimentales que reporten una caída de presión radial dentro de un lecho empacado con las dimensiones similares a las de este trabajo para obtener la componente de velocidad radial, razón por la que la componente de velocidad radial se desprecia en los modelos ${ }^{1,2,5,19,23,38-40}$, sin embargo, existen estudios de $\mathrm{CFD}^{41,42}$ que sugieren el uso de la componente axial y radial de la velocidad para capturar el transporte de calor convectivo y los cambios en el transporte de calor cercanos a la pared, en donde las componentes de velocidad axial y radial se obtienen mediante el uso de CFD. En estos estudios, se ha visto que la componente de velocidad radial puede alcanzar desde un $10 \%$ hasta un $20 \%$ el valor de la velocidad en dirección axial.

Aunado a lo anterior, en este trabajo se toman en consideran tres casos de estudio, en donde la componente de la velocidad radial tiene los siguientes valores: 10, 15 y 20\% de la componente de velocidad axial. Esto con la finalidad de observar el efecto de la velocidad radial en la transferencia de calor en el lecho.

\section{Velocidad radial $10 \%$ de la velocidad axial}

Los ajustes del modelo pseudo-homogéneo en estado transitorio considerando la aproximación de la capa límite y las componentes de la velocidad, axial y radial, en donde la componente de la velocidad radial tiene un valor del $10 \%$ de la velocidad axial, se muestran en las figuras 4.17 y 4.18. El valor estimado de $\mathrm{k}_{\text {eff,r }}$ es de $0.811383 \mathrm{~W} / \mathrm{m} \mathrm{K}$, el cual resulta ser un $25 \%$ mayor al obtenido cuando se desprecia la componente radial de la velocidad en la aproximación de la capa límite, el I.C. del 95\% es 0.811380-0.811386, y su diferencia con

respecto a las $\mathrm{k}_{\mathrm{eff}, \mathrm{r}}$ obtenidas de las correlaciones reportadas en la literatura, ver Figura 2.4, varía entre el $16 \%$ y $82 \%$. Para la estimación de keff,r se tomaron en cuenta todas las temperaturas axiales y radiales observadas. 


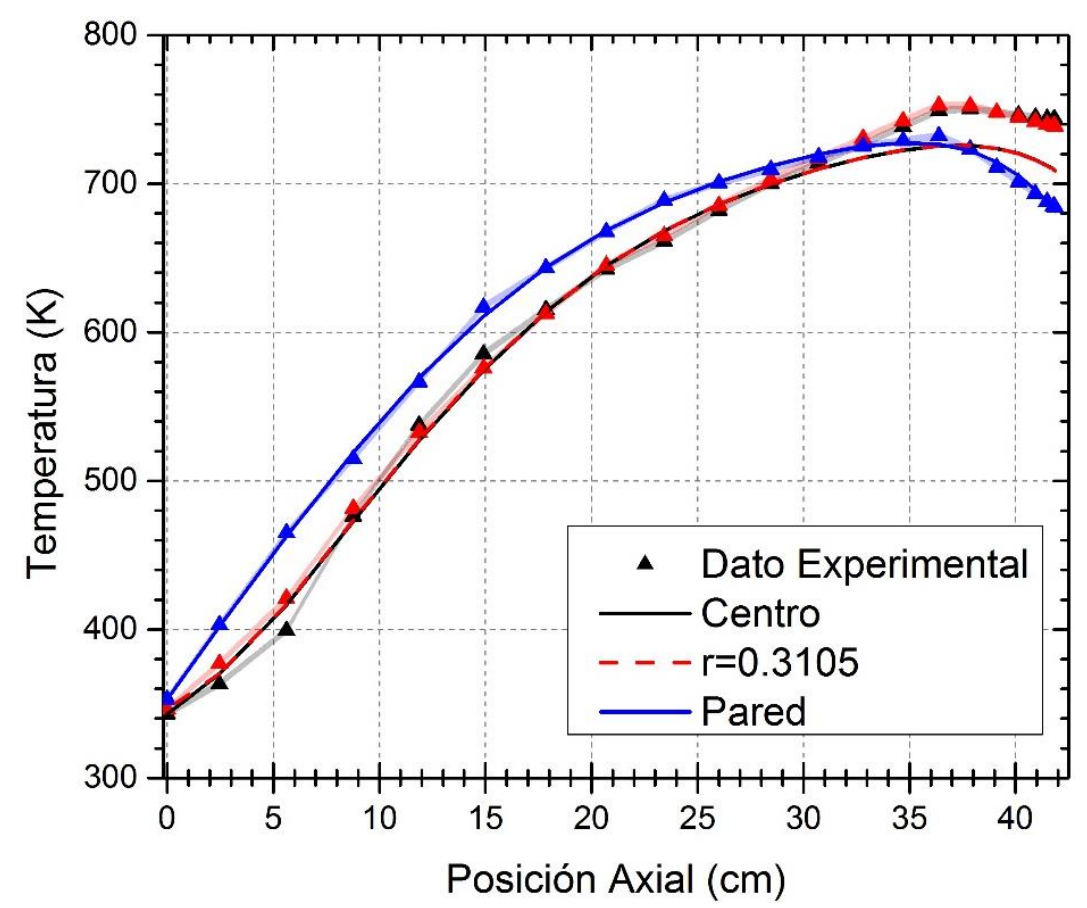

Figura 4.17. Perfil axial de temperatura en el lecho no isotérmico no adiabático con aproximación de la capa límite y empleando el $10 \%$ de $\mathrm{v}_{\mathrm{z}}$ en $\mathrm{v}_{\mathrm{r}}, \mathrm{Re}_{\mathrm{p}}=163.36$,

a 3 posiciones radiales diferentes; ( $\mathbf{\Lambda}$ ) dato experimental, (-) modelo.

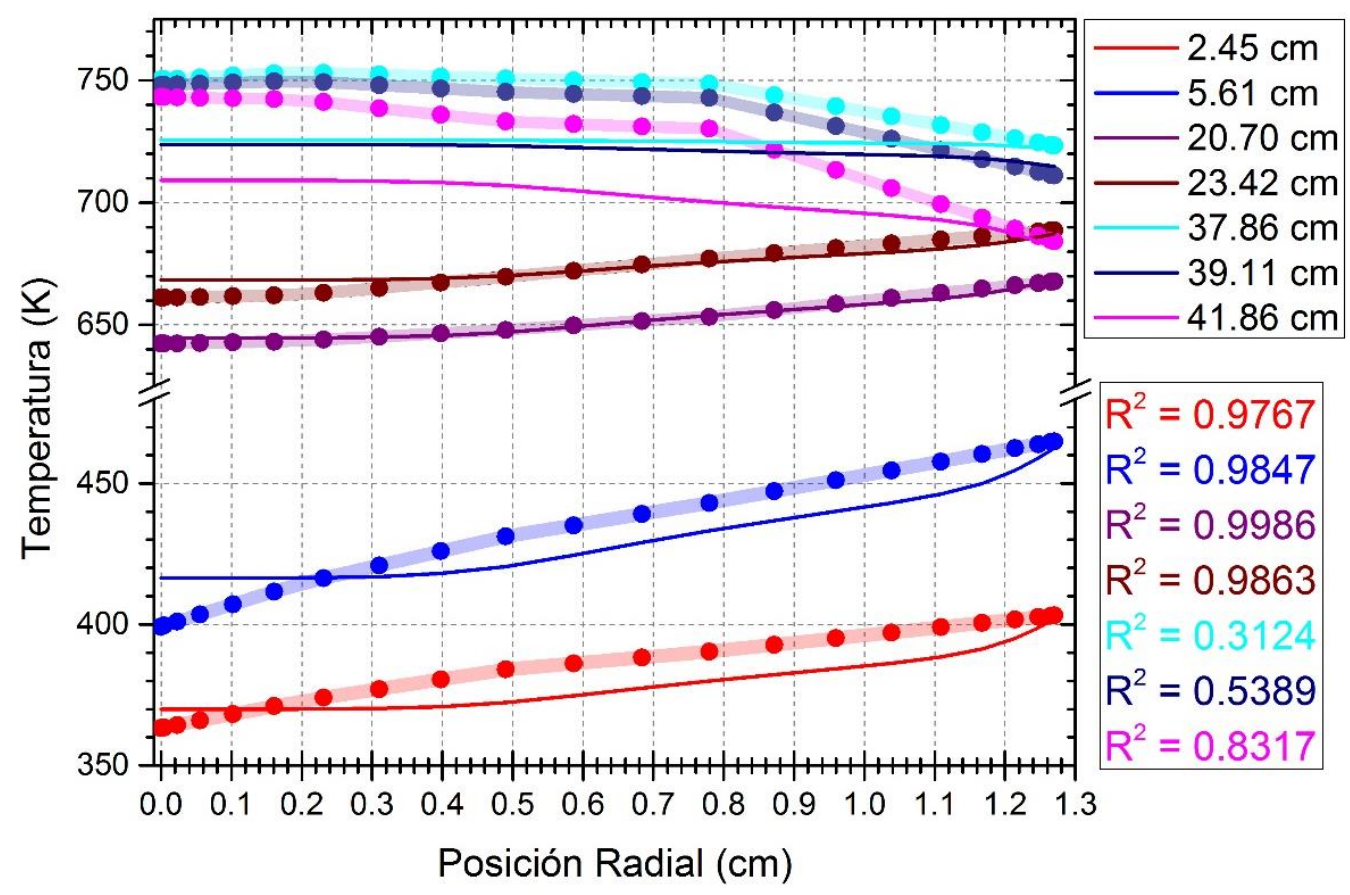

Figura 4.18. Perfil radial de temperatura en el lecho no isotérmico no adiabático con aproximación de la capa límite y empleando el $10 \%$ de $\mathrm{v}_{\mathrm{z}}$ en $\mathrm{v}_{\mathrm{r}}, \mathrm{Re}_{\mathrm{p}}=163.36$,

a 7 posiciones axiales diferentes; ( $\mathbf{\Lambda})$ dato experimental, $(-)$ modelo. 
El coeficiente de correlación, $\mathrm{R}^{2}$, de los perfiles de temperatura axial en el centro del lecho es de 0.9985 y en la pared de 0.9999 , mientras que el $\mathrm{R}^{2}$ en los perfiles radiales de temperatura en la salida del lecho, $\mathrm{z}=41.86 \mathrm{~cm}, 39.11 \mathrm{~cm}$ y $37.86 \mathrm{~cm}$, son de $0.8317,0.5389$ y 0.3124 . Los perfiles de temperatura obtenidos estadísticamente muestran una ligera mejora a los perfiles de temperatura de las dos primeras aproximaciones que desprecian la velocidad radial, Figura 4.11-4.14. Sin embargo, sigue habiendo una mala representación de los perfiles de temperatura en la salida del lecho y en la región central del lecho. Lo anterior puede indicar que un $10 \%$ del valor de la velocidad axial en la velocidad radial tiene poco efecto en la predicción de los perfiles de temperatura, por lo que más adelante se mostrará el efecto de un valor mayor en la $v_{r}$.

\section{Velocidad radial $15 \%$ de la velocidad axial}

En las figuras 4.19 y 4.20 se presentan los ajustes del modelo de transferencia de calor pseudo-homogéneo en estado transitorio, en donde la componente radial de la velocidad tiene el valor del $15 \%$ de la velocidad axial. El valor estimado de $\mathrm{k}_{\text {eff, }}$ es de $0.893642 \mathrm{~W} / \mathrm{m} \mathrm{K}$, el cual, de acuerdo a los fundamentos, crece en función de un aumento la velocidad radial, favoreciendo una transferencia de calor en dirección radial. El I.C. del 95\% de la $\mathrm{k}_{\mathrm{eff}, \mathrm{r}}$ estimada es de $0.893635-0.893650$, mostrando una diferencia del $24 \%$ al $84 \%$ con respecto a los valores reportados en otras investigaciones y con otras aproximaciones utilizadas en esta investigación. El $\mathrm{R}^{2}$ de los perfiles axiales de temperatura en el centro del lecho es de 0.9985 y en la pared es de 0.9999 , mientras que el $R^{2}$ de los perfiles radiales de temperatura en la salida del lecho en $\mathrm{z}=41.86 \mathrm{~cm}, 39.11 \mathrm{~cm}$ y $37.86 \mathrm{~cm}$ son de $0.8234,0.5271$ y 0.3096 . Para la estimación de $\mathrm{k}_{\mathrm{eff}, \mathrm{r}} \mathrm{se}$ tomaron en cuenta todas las temperaturas axiales y radiales observadas.

Estos resultados no varían estadísticamente con respecto de los obtenidos cuando se considera el $15 \%$ de $\mathrm{v}_{\mathrm{z}}$ en $\mathrm{v}_{\mathrm{r}}$ o cuando se considera la aproximación de la capa límite sin el efecto de la $v_{r}$. 


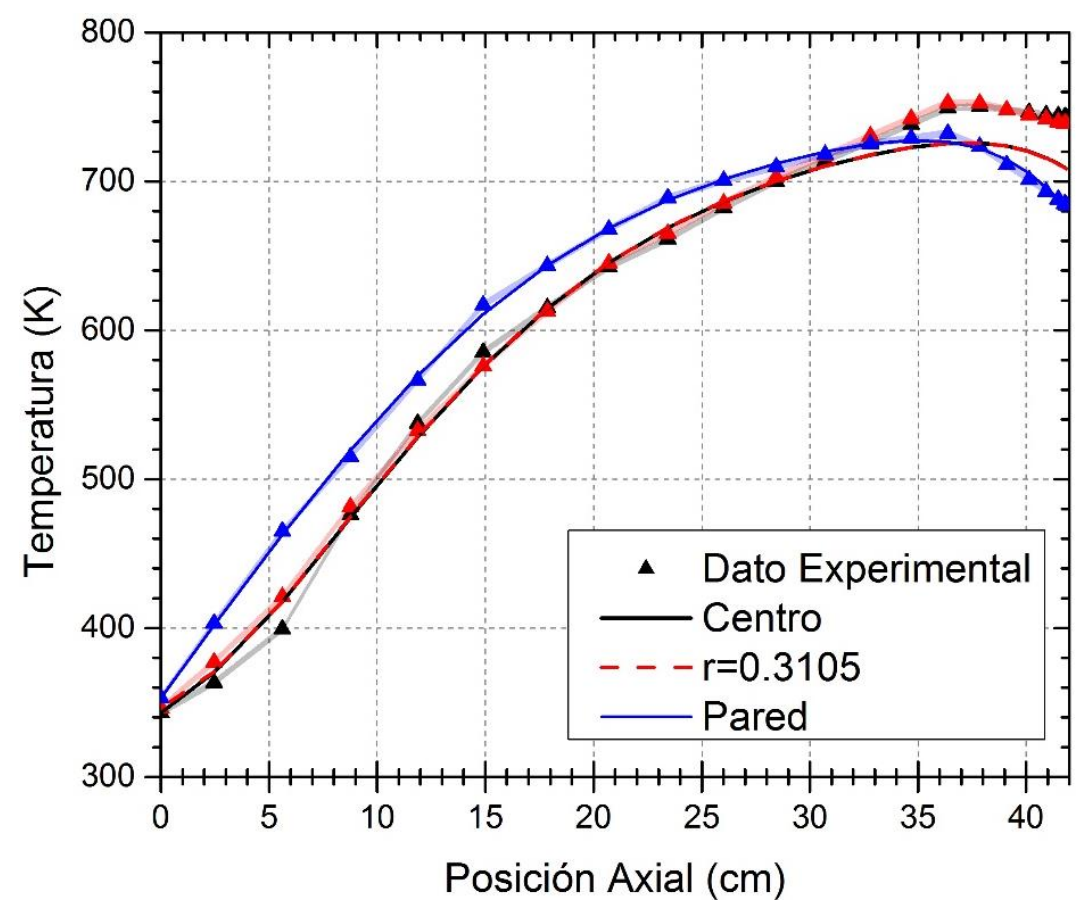

Figura 4.19. Perfil axial de temperatura en el lecho no isotérmico no adiabático con aproximación de la capa límite y empleando el $15 \%$ de $\mathrm{v}_{\mathrm{z}}$ en $\mathrm{v}_{\mathrm{r}}, \mathrm{Re}_{\mathrm{p}}=163.36$,

a 3 posiciones radiales diferentes; ( $\mathbf{\Lambda})$ dato experimental, (-) modelo.

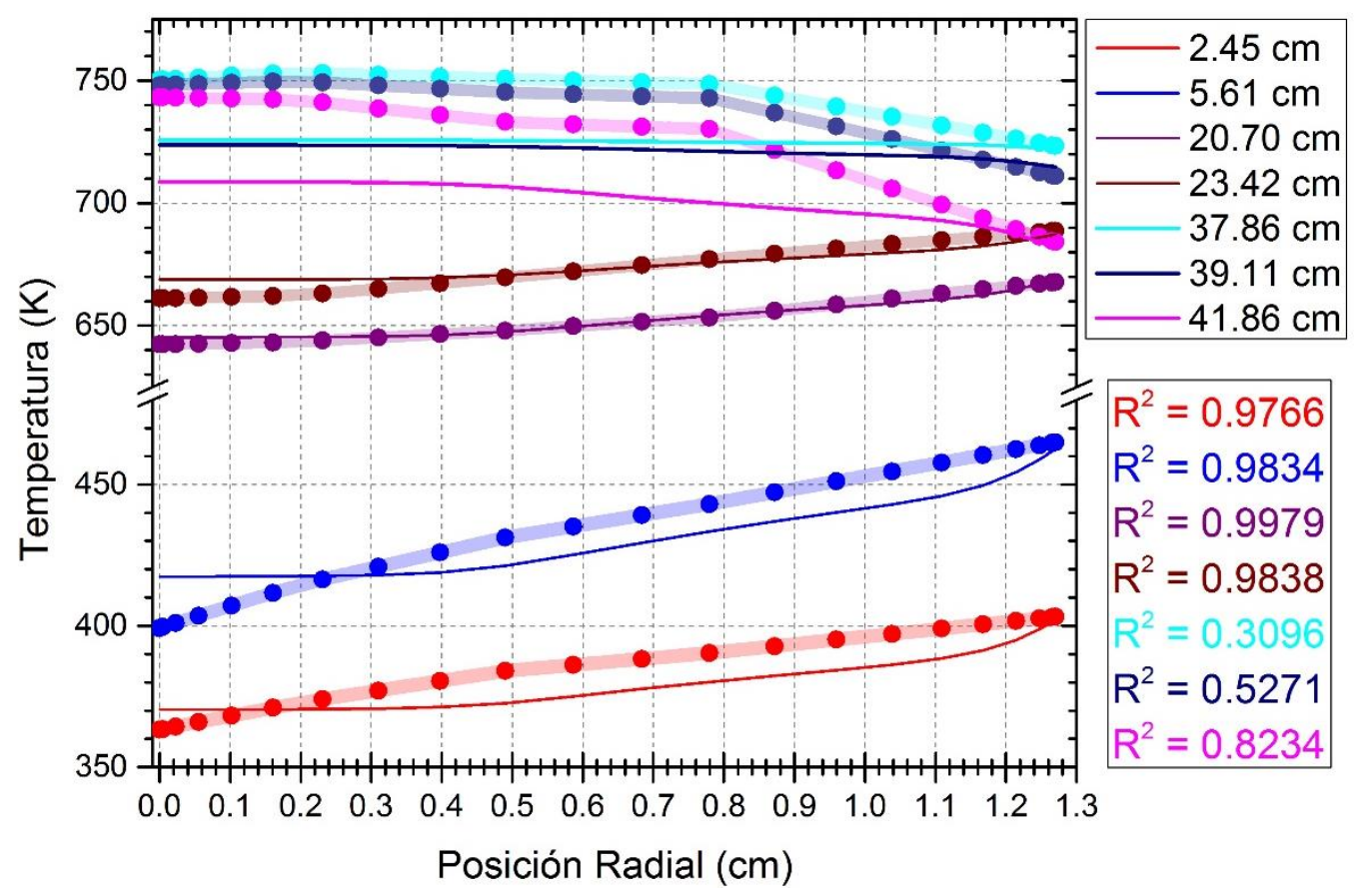

Figura 4.20. Perfil radial de temperatura en el lecho no isotérmico no adiabático con aproximación de la capa límite y empleando el $15 \%$ de $\mathrm{v}_{\mathrm{z}}$ en $\mathrm{v}_{\mathrm{r}}, \mathrm{Re}_{\mathrm{p}}=163.36$,

a 7 posiciones axiales diferentes; ( $\mathbf{\Lambda})$ dato experimental, $(-)$ modelo. 
Tomando en cuenta que la componente de velocidad radial no puede tener un valor mayor al $30 \%$ de la velocidad axial, y por la manera en que la $\mathrm{v}_{\mathrm{r}}$ afecta la transferencia de calor en el lecho, se cree que el considerar la componente de velocidad radial no soluciona del todo la descripción de los mecanismos de transferencia de calor en el lecho, esencialmente a la salida del lecho y en su región central, por lo que más adelante se evaluará la $\mathrm{v}_{\mathrm{r}}$ considerando su valor como el $20 \%$ de $\mathrm{v}_{\mathrm{z}}$, considerándolo como el caso en donde la componente de velocidad radial obtiene su máximo valor en el lecho.

\section{Velocidad radial $20 \%$ de la velocidad axial}

Las figuras 4.21 y 4.22 muestran los perfiles de temperatura obtenidos del ajuste del modelo no isotérmico y no adiabático pseudo-homogéneo en estado transitorio considerando a $\mathrm{v}_{\mathrm{r}}$ con un valor del $20 \%$ de $\mathrm{v}_{\mathrm{z}}$. Para la estimación de $\mathrm{k}_{\mathrm{eff} f \mathrm{r}}$ se tomaron en cuenta todas las temperaturas axiales y radiales observadas. El valor estimado de $\mathrm{k}_{\text {eff,r }}$ es de $0.972561 \mathrm{~W} / \mathrm{m} \mathrm{K}$, valor que se difiere entre un $30 \%$ y $85 \%$ de las $\mathrm{k}_{\text {eff,r }}$ reportadas en la literatura y obtenidas con las otras aproximaciones presentadas en este estudio. El I.C. del 95\% es $0.972559-0.972564$. El coeficiente de correlación de los perfiles de temperatura axial en el centro es de 0.9985 y en la pared es de 0.9999, y para el perfil de temperatura radial a la salida del lecho, $\mathrm{z}=41.86$ $\mathrm{cm}, 39.11 \mathrm{~cm}$ y $37.86 \mathrm{~cm}$, el $\mathrm{R}^{2}$ es de $0.8160,0.5163$ y 0.3006 , respectivamente.

Esto muestra un ligero deterioro en la predicción del perfil radial de temperatura a la salida del lecho, en comparación de considerar $10 \%$ o $15 \%$ de $\mathrm{v}_{\mathrm{z}}$ en $\mathrm{v}_{\mathrm{r}}$, lo cual nos indica que el considerar la componente de velocidad radial como un $20 \%$ de $\mathrm{v}_{\mathrm{z}}$ puede ser incorrecto al momento de tomar en cuenta la convección radial en los mecanismos de transferencia de calor en la región central y cerca de la pared del lecho. Todo parece indicar que el considerar una $\mathrm{v}_{\mathrm{r}}$ que tenga un valor menor al 15\% de $\mathrm{v}_{\mathrm{z}}$, y una keff,r en función de la posición radial, es decir, como valores pseudo locales, pudiese permitir describir los mecanismos de transferencia de calor en un lecho como el que se estudia en este trabajo. 


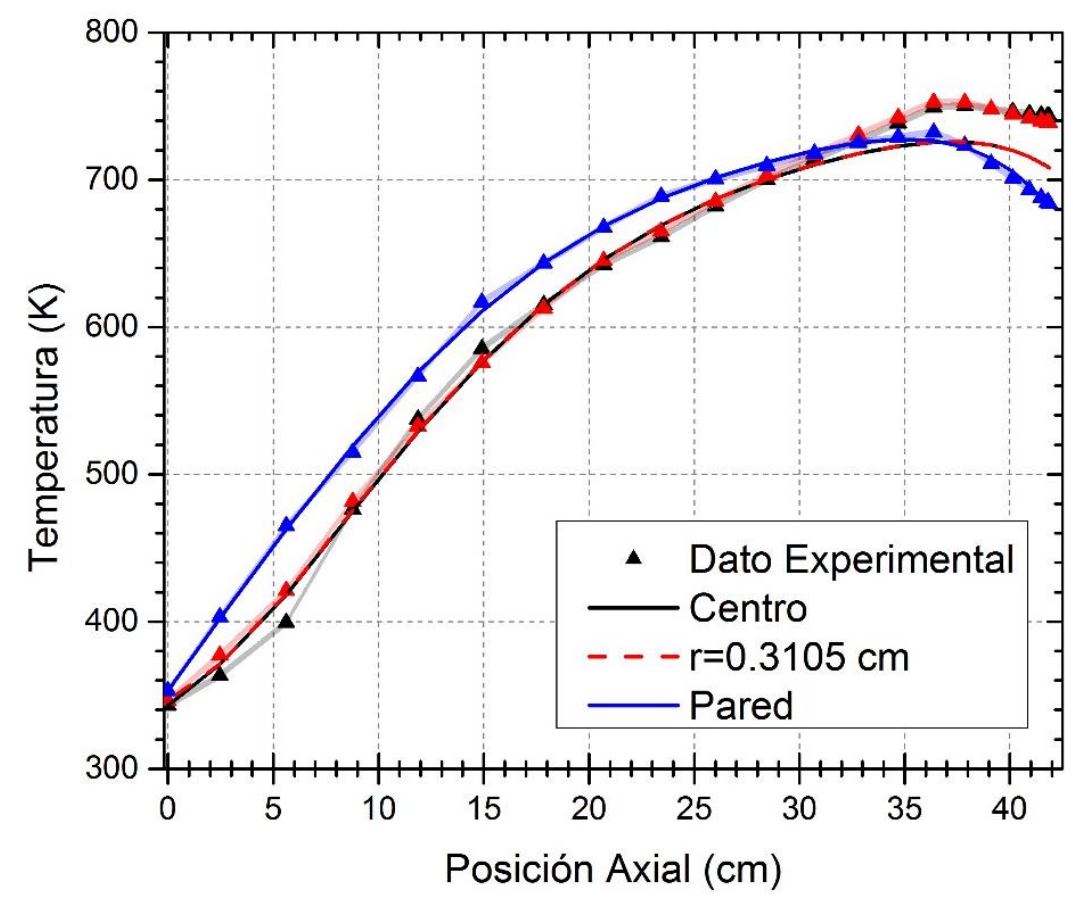

Figura 4.21. Perfil axial de temperatura en el lecho no isotérmico no adiabático con aproximación de la capa límite y empleando el $20 \%$ de $\mathrm{v}_{\mathrm{z}}$ en $\mathrm{v}_{\mathrm{r}}, \mathrm{Re}_{\mathrm{p}}=163.36$,

a 3 posiciones radiales diferentes; ( $\mathbf{\Lambda})$ dato experimental, (-) modelo.

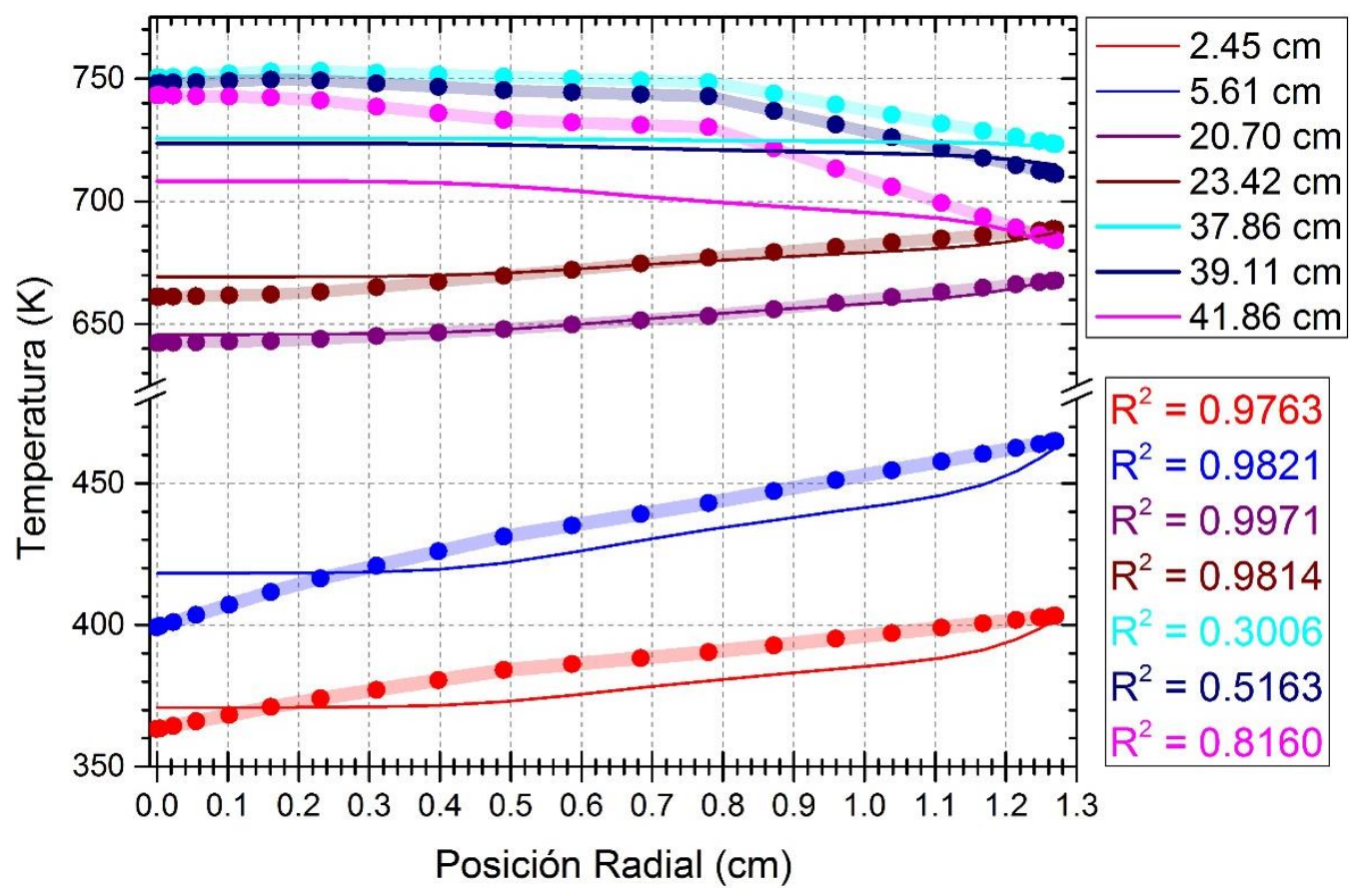

Figura 4.22. Perfil radial de temperatura en el lecho no isotérmico no adiabático con aproximación de la capa límite y empleando el $20 \%$ de $\mathrm{v}_{\mathrm{z}}$ en $\mathrm{v}_{\mathrm{r}}, \mathrm{Re}_{\mathrm{p}}=163.36$,

a 7 posiciones axiales diferentes; ( $\mathbf{\Lambda})$ dato experimental, $(-)$ modelo. 


\subsubsection{Velocidad radial $10 \%$ de la velocidad axial, estimación con datos del centro del lecho}

El último caso a tratar en este trabajo es el mismo que se presentó cuando se supuso que la $\mathrm{v}_{\mathrm{r}}$ tiene el $10 \%$ del valor de la $\mathrm{v}_{\mathrm{z}}$, con la diferencia de que la estimación del parámetro keff,r ahora sólo tomará en cuenta los datos observados en el centro del lecho. Lo anterior con el fin de esclarecer la importancia de tomar en cuenta la mayor cantidad de información dentro del lecho.

Los ajustes del modelo de transferencia de calor pseudo-homogéneo en estado transitorio, en donde la componente radial de la velocidad tiene el valor del $10 \%$ de la velocidad axial, empleando la aproximación de la capa límite, y estimando conociendo únicamente los datos del centro del lecho se muestran en las figuras 4.23 y 4.24 .

La $\mathrm{k}_{\text {eff,r }}$ estimada en este caso es de $0.7492 \mathrm{~W} / \mathrm{m} \mathrm{K}$, dando una diferencia del $7 \%$ al valor estimado cuando se toma en cuenta todo el campo de temperatura dentro del lecho para hacer la estimación de este parámetro. El I.C. del $95 \%$ es $0.5270-0.9714$, el $\mathrm{R}^{2}$ del perfil axial en

el centro del lecho es de 0.9986 , y el $\mathrm{R}^{2}$ del perfil axial en la pared es de 0.9999 , mientras que el $\mathrm{R}^{2}$ del perfil radial a la salida del lecho, a $\mathrm{z}=41.86 \mathrm{~cm} 39.11 \mathrm{~cm}$ y $37.86 \mathrm{~cm}$, son de $0.8522,0.5450$ y 0.3058 . A pesar de que estadísticamente no existe una diferencia apreciable en el coeficiente de correlación, lo anterior muestra el efecto del número y tipo de observaciones en la caracterización de la transferencia de calor en un lecho no isotérmico no adiabático, teniendo una diferencia del $7.66 \%$ entre la keff,r estimada aquí y aquella obtenida utilizando todo el campo de temperatura observado. 


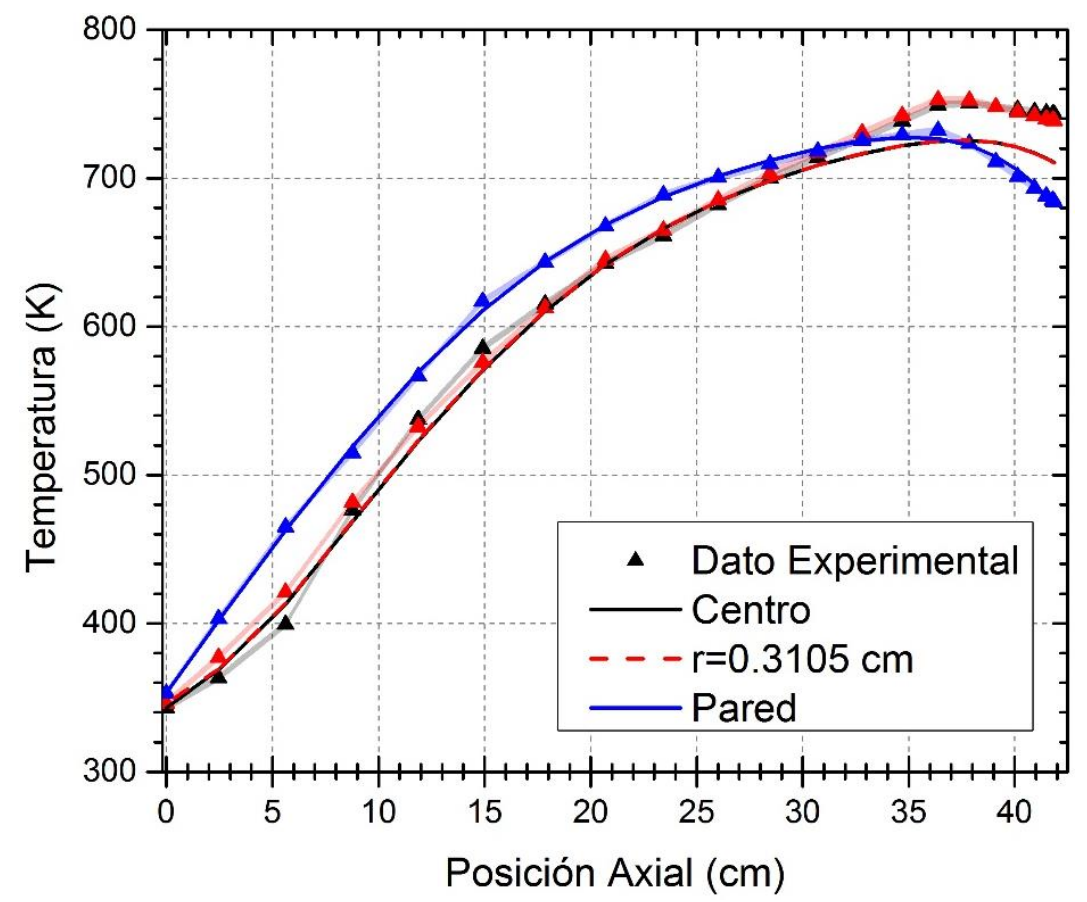

Figura 4.23. Perfil axial de temperatura en el lecho no isotérmico no adiabático con aproximación de la capa límite, empleando el $10 \%$ de $\mathrm{v}_{\mathrm{z}}$ en $\mathrm{v}_{\mathrm{r}}$, estimación con datos del centro del lecho, $\mathrm{Re}_{\mathrm{p}}=163.36$, a 3 posiciones radiales diferentes.

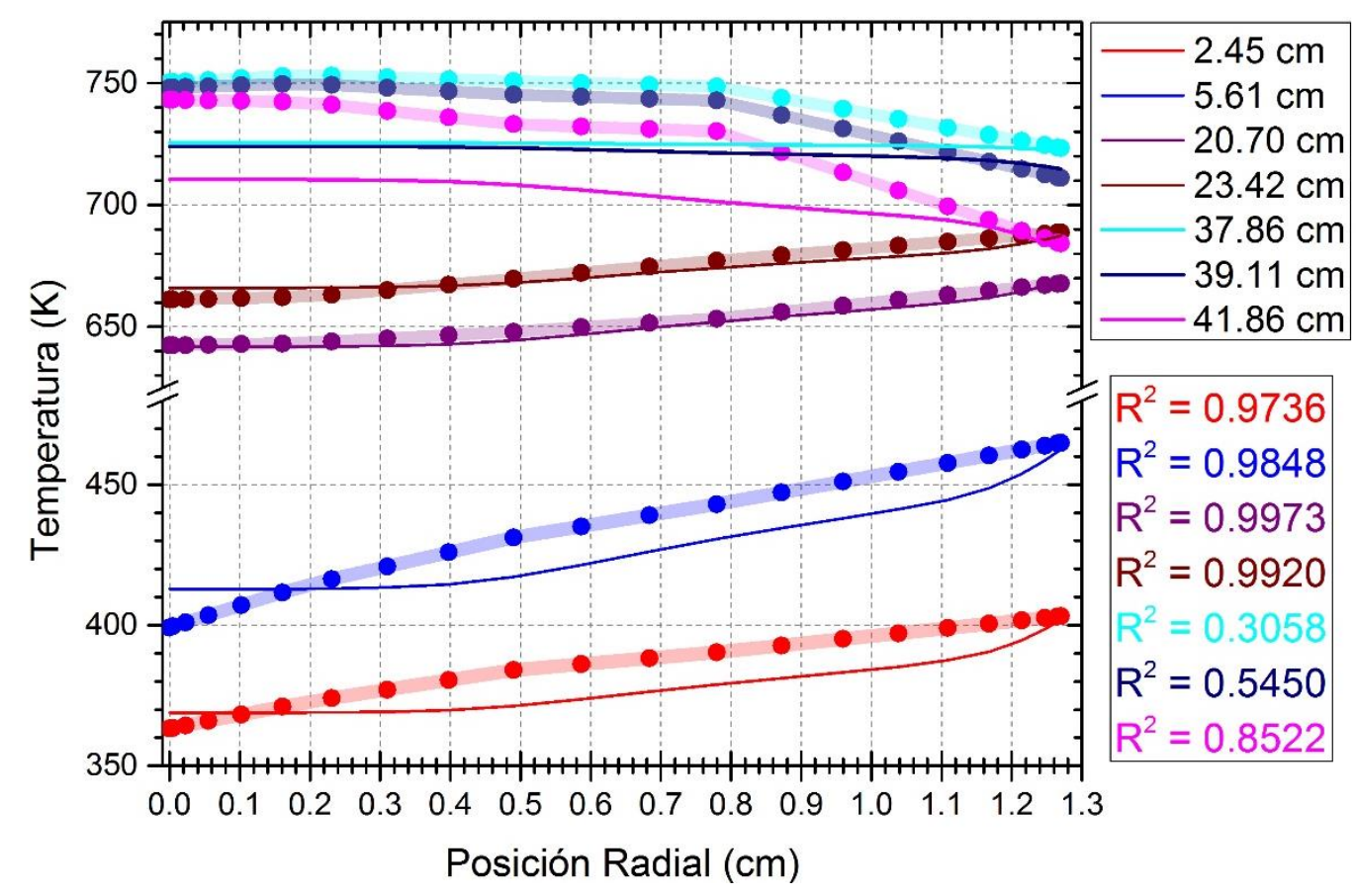

Figura 4.24. Perfil radial de temperatura en el lecho no isotérmico no adiabático con aproximación de la capa límite, empleando el $10 \%$ de $\mathrm{v}_{\mathrm{z}}$ en $\mathrm{v}_{\mathrm{r}}$, estimación con datos del centro del lecho, $\operatorname{Re}_{\mathrm{p}}=163.36$, a 7 posiciones axiales diferentes. 


\subsubsection{Comparación entre las aproximaciones}

Para dar una perspectiva general de los resultados obtenidos con cada una de las aproximaciones que se evaluaron en este estudio, se comparan y analizan los perfiles de temperatura, axiales y radiales, descritos por estas.

\section{Perfiles radiales de temperatura}

En la Figura 4.25 se presenta una comparación de los perfiles radiales de temperatura obtenidos con las distintas aproximaciones en la posición axial z $=2.5 \mathrm{~cm}$ y z $=5.61 \mathrm{~cm}$. De igual manera, en la Figura 4.26 se presenta una comparación de los perfiles radiales de temperatura obtenido de las diferentes aproximaciones en la posición axial $\mathrm{z}=20.7 \mathrm{~cm} \mathrm{y}$ $\mathrm{z}=23.42 \mathrm{~cm}$.

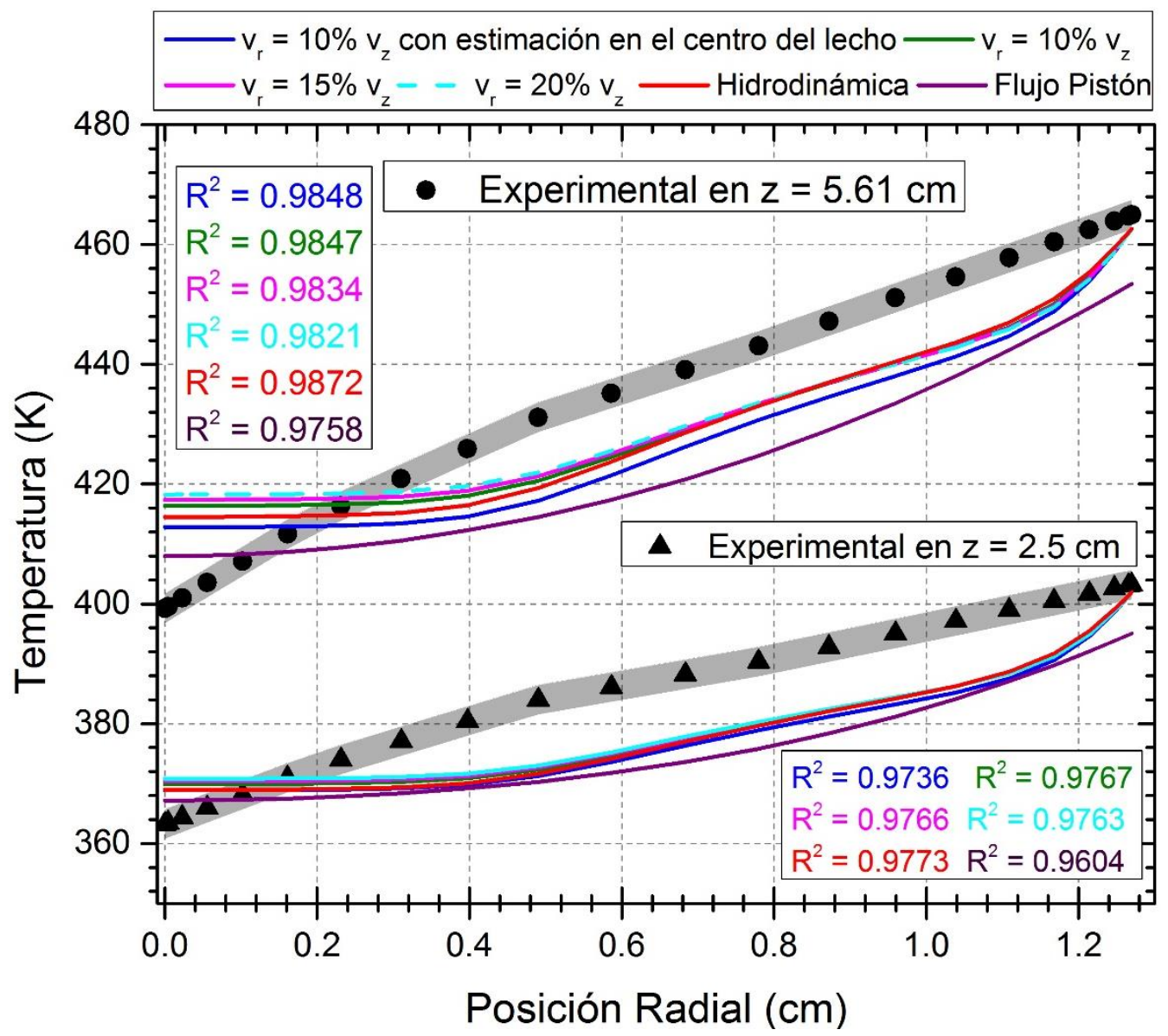

Figura 4.25. Comparación de los perfiles radiales de temperatura en el lecho no isotérmico no adiabático obtenidos con las diferentes aproximaciones, $\mathrm{Re}_{\mathrm{p}}=163.36, \mathrm{z}=2.5 \mathrm{~cm}$ y $5.61 \mathrm{~cm}$. 


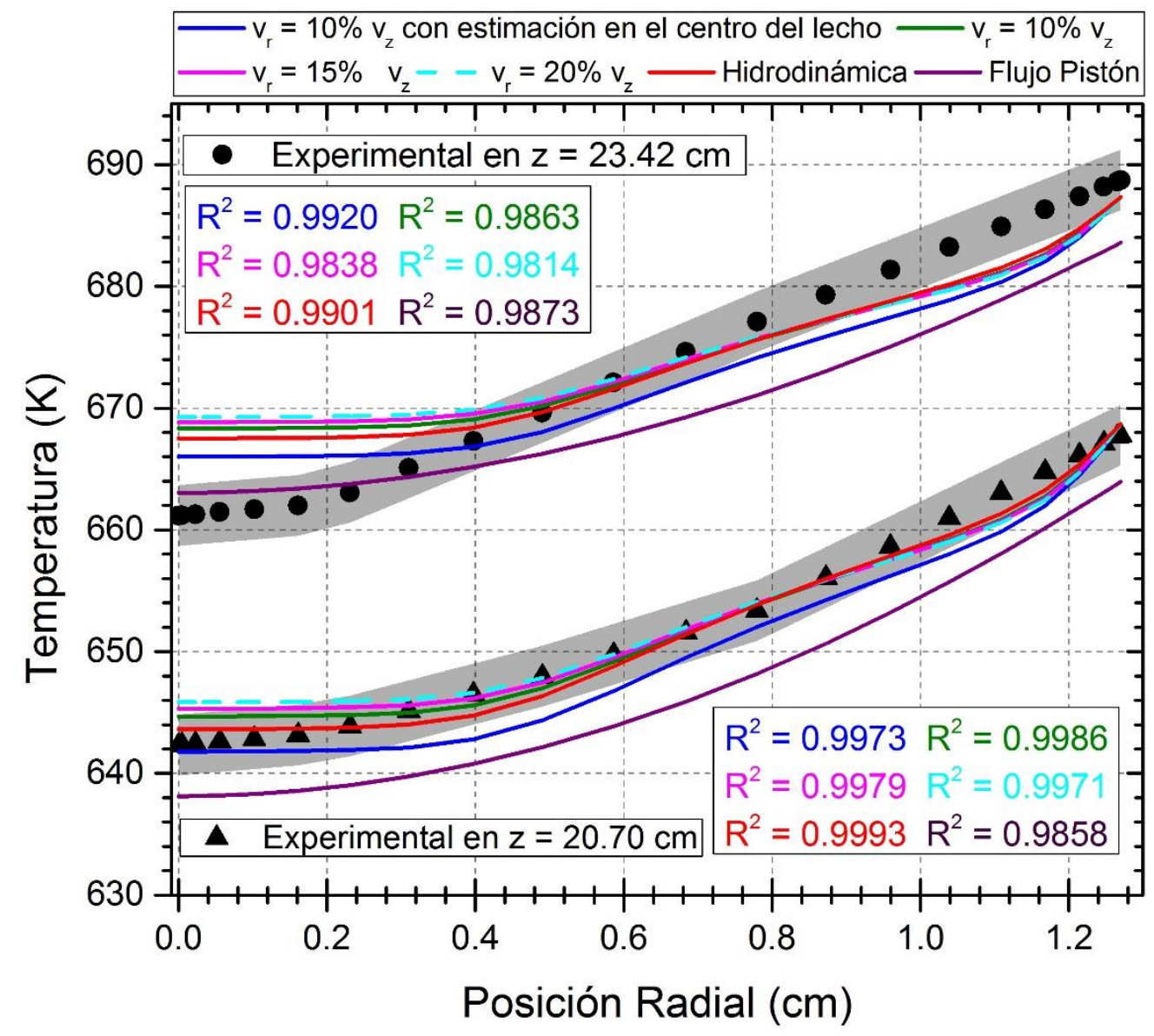

Figura 4.26. Comparación de los perfiles radiales de temperatura en el lecho no isotérmico no adiabático obtenidos con las diferentes aproximaciones, $\operatorname{Re}_{\mathrm{p}}=163.36, \mathrm{z}=20.70 \mathrm{~cm}$ y $23.42 \mathrm{~cm}$.

Estas comparaciones reflejan que el uso de la componente de la velocidad radial disminuye el gradiente de temperatura en el lecho. Sin importar si se emplea un 10\%, 15\% o $20 \%$ de la $\mathrm{v}_{\mathrm{z}}$ en $\mathrm{v}_{\mathrm{r}}$, se mantiene un perfil radial de temperatura muy similar para todos los casos. A diferencia del flujo pistón, la mayoría de los modelos parecen tener mejor predicción de los perfiles de temperatura radiales a estas posiciones axiales, ver coeficientes de correlación en las Figuras 4.25 y 4.26. Se observa que el modelo que estima la keff,r a partir de los datos solamente en el centro del lecho predice mejor la temperatura en el centro del lecho, mientras que el modelo que estimó la k eff,r $_{\mathrm{r}}$ utilizando observaciones axiales y radiales de temperatura predice mejor la transferencia de calor radial. Lo que nos sugiere que la keff,r es un parámetro que es función de la posición radial, y debe considerarse así para tener una mejor descripción de los mecanismos de transferencia de calor en el lecho no isotérmico no adiabático. 


\section{Perfiles axiales de temperatura}

Como se observó anteriormente, el empleo de un 10\%, 15\% y 20\% de $\mathrm{v}_{\mathrm{z}}$ en $\mathrm{v}_{\mathrm{r}}$ no genera una diferencia apreciable entre los perfiles predichos. Por lo que para la comparación de perfiles axiales de temperatura en el lecho no isotérmico no adiabático, se utiliza el caso cuando $\mathrm{v}_{\mathrm{r}}$ tiene el valor del 10\% de $\mathrm{v}_{\mathrm{z}}$. Las figuras 4.27, 4.28 y 4.29 muestran las comparaciones de los perfiles de temperatura axiales con las diferentes aproximaciones en tres posiciones radiales; centro del lecho, pared interna del lecho y $\mathrm{r}=0.3106 \mathrm{~cm}$.

Nuevamente, se observa que la mayoría de los modelos tienen una buena predicción de los perfiles de temperatura axiales, inclusive la aproximación que considera el flujo pistón. Sin embargo, como se había mencionado anteriormente, en la salida del lecho se predice una caída de temperatura que es diferente a la que es observada.

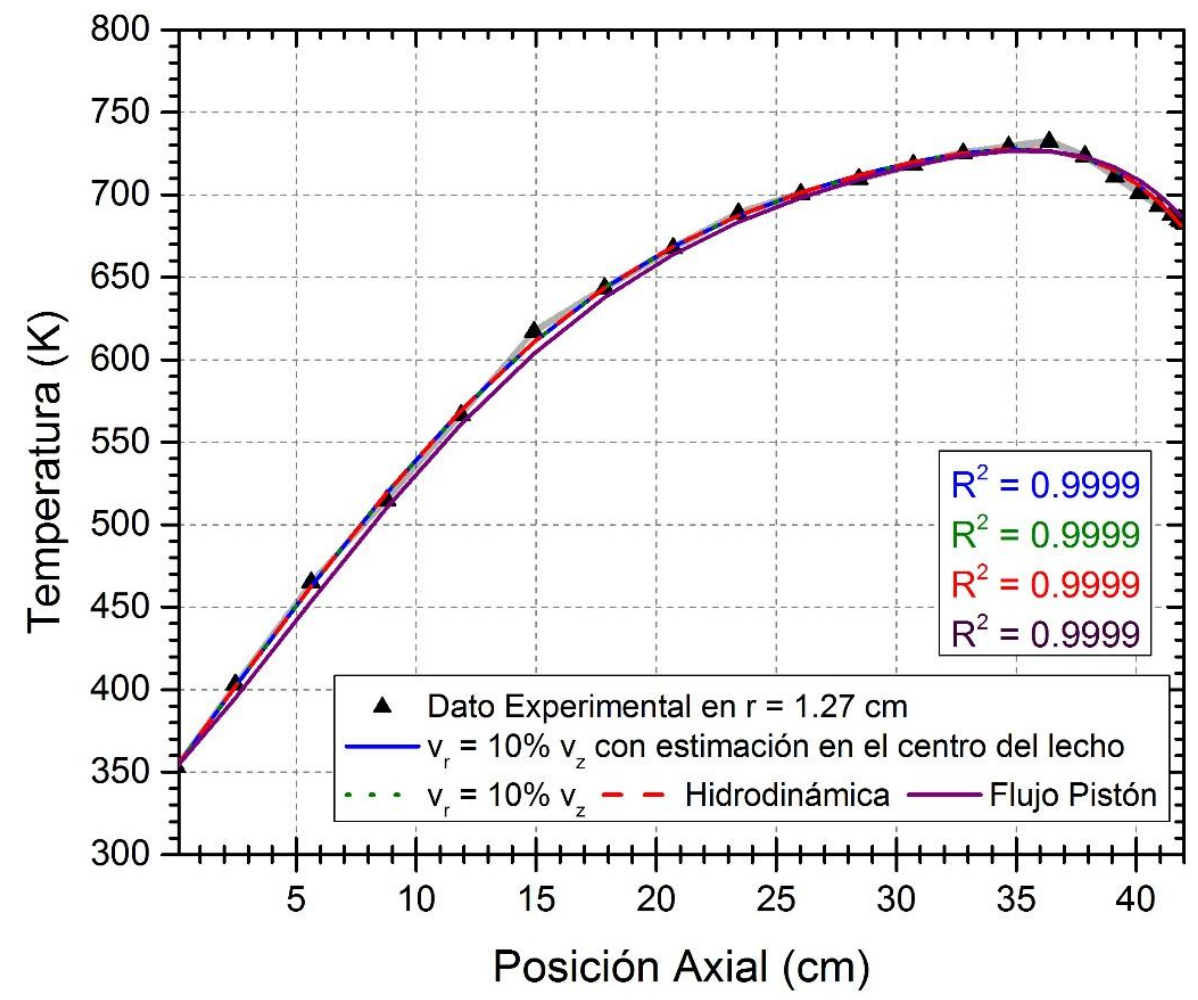

Figura 4.27. Comparación de los perfiles axiales de temperatura en el lecho no isotérmico no adiabático obtenidos con las diferentes aproximaciones, $\mathrm{Re}_{\mathrm{p}}=163.36, \mathrm{r}=1.27 \mathrm{~cm}$. 


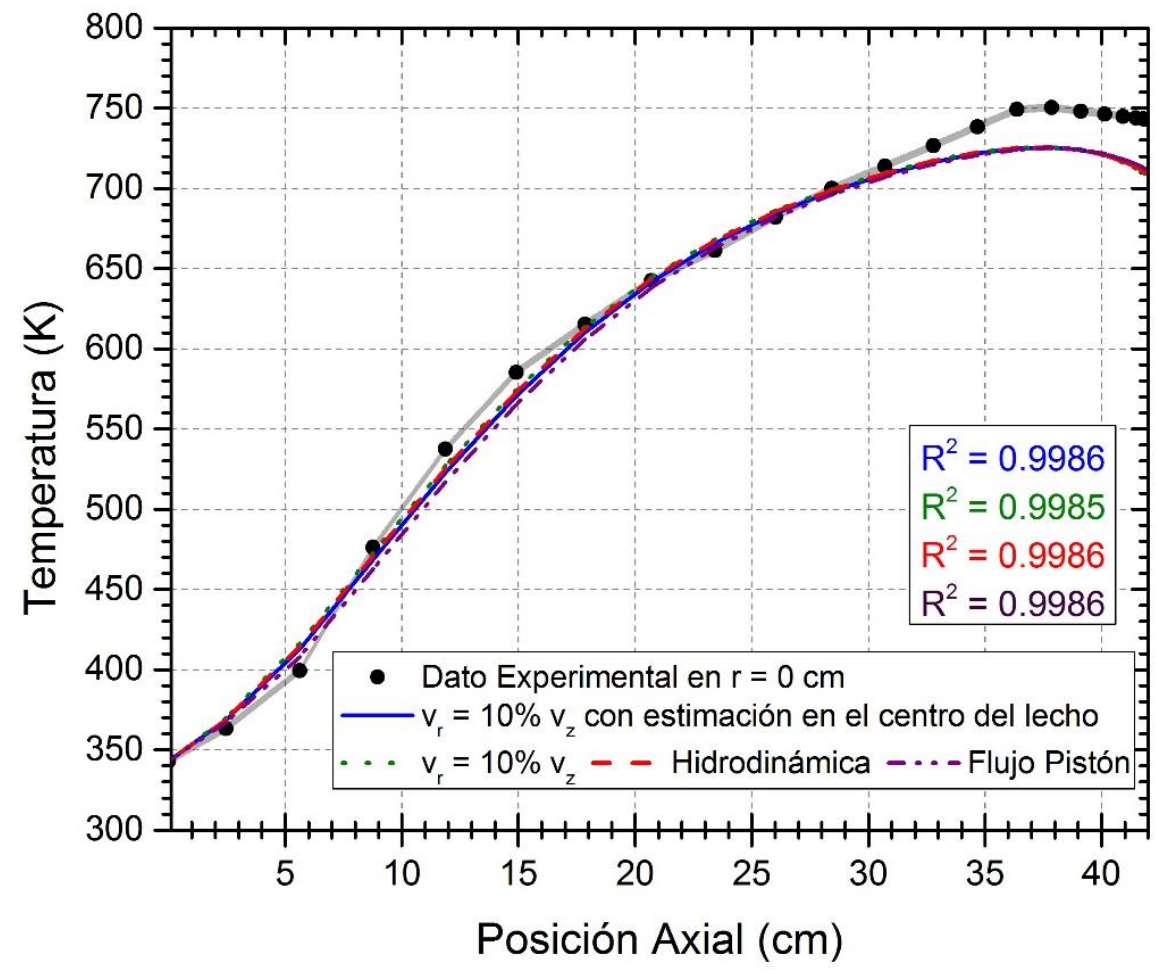

Figura 4.28. Comparación de los perfiles axiales de temperatura en el lecho no isotérmico no adiabático obtenidos con las diferentes aproximaciones, $R_{\mathrm{p}}=163.36, \mathrm{r}=0 \mathrm{~cm}$.

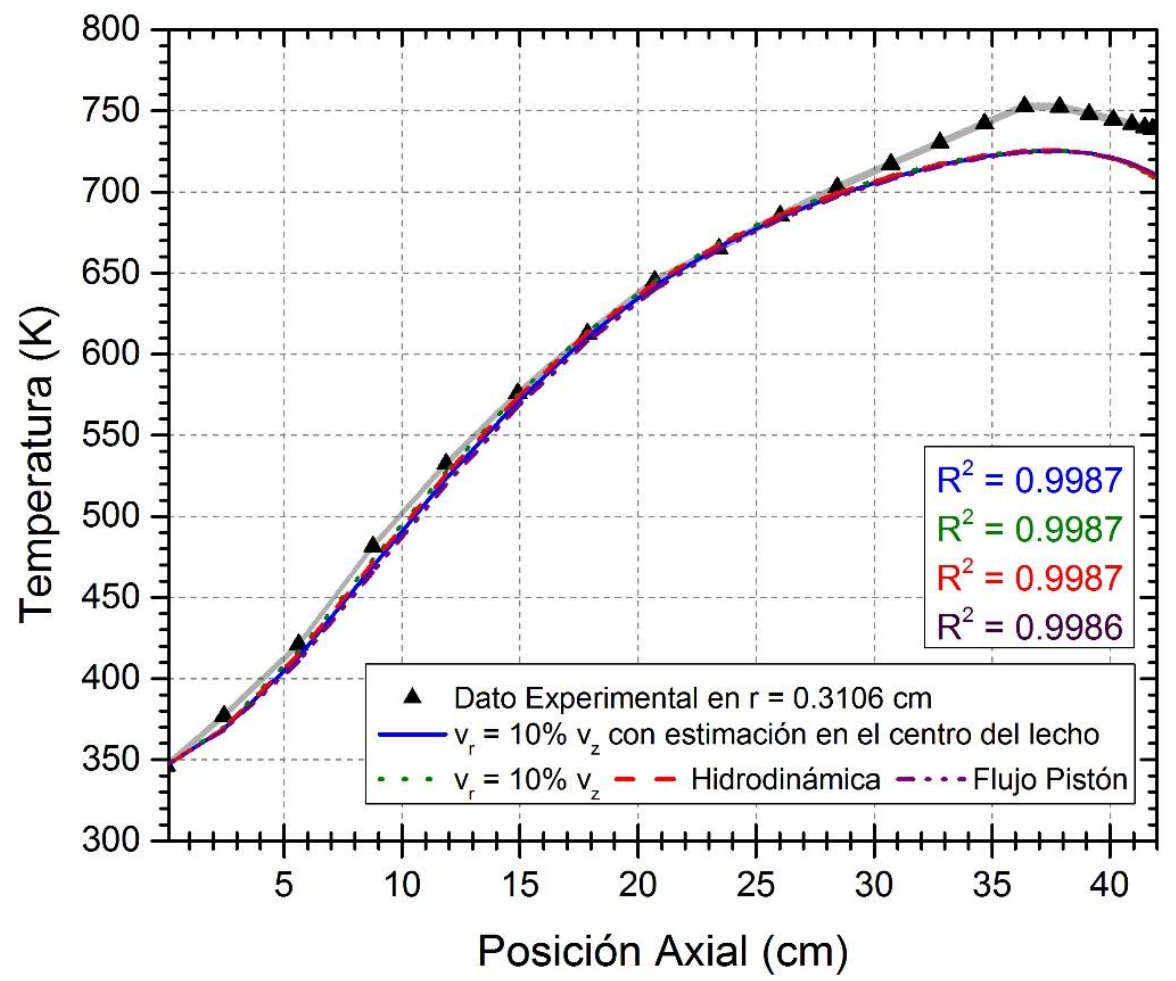

Figura 4.29. Comparación de los perfiles axiales de temperatura en el lecho no isotérmico no adiabático obtenidos con las diferentes aproximaciones, $\mathrm{Re}_{\mathrm{p}}=163.36$, en $\mathrm{r}=0.3106 \mathrm{~cm}$. 


\subsubsection{Evaluación de la transferencia de calor a $\operatorname{Re}_{\mathrm{p}}=40.83$ y 81.67}

Para la descripción de la transferencia de calor en el lecho con calentamiento en la pared a un $\operatorname{Re}_{\mathrm{p}}$ de 40.83 y 81.67 se utiliza la aproximación de la capa límite y las componentes de la velocidad, axial y radial, en donde $\mathrm{v}_{\mathrm{r}}$ es el $10 \%$ de $\mathrm{v}_{\mathrm{z}}$. Cabe señalar que el área sombreada alrededor de los datos experimentales, en las figuras, indican el $\pm 2.5 \mathrm{~K}$ de error experimental, y que para la estimación de $\mathrm{k}_{\text {eff,r }}$ se tomaron en cuenta todas las temperaturas axiales y radiales observadas.

\section{$\operatorname{Re}_{\mathrm{p}}=\mathbf{4 0 . 8 3}$}

Los perfiles de temperatura axiales y radiales del lecho con calentamiento de pared a un Rep de 40.83 se muestran en las figuras 4.30 y 4.31 . El valor estimado de $k_{\text {eff,r }}$ es de $0.3076 \mathrm{~W} / \mathrm{m}$ K, y sus I.C. del $95 \%$ son 0.3075 - 0.3077. A diferencia de los perfiles de temperatura obtenidos cuando el $\operatorname{Re}_{\mathrm{p}}$ es de 163.36, el punto máximo de temperatura, o punto caliente, se encuentra casi en el centro de la longitud del lecho empacado, lo cual indica que el punto caliente se va desplazando hacia la salida del lecho conforme aumenta el flujo del fluido.

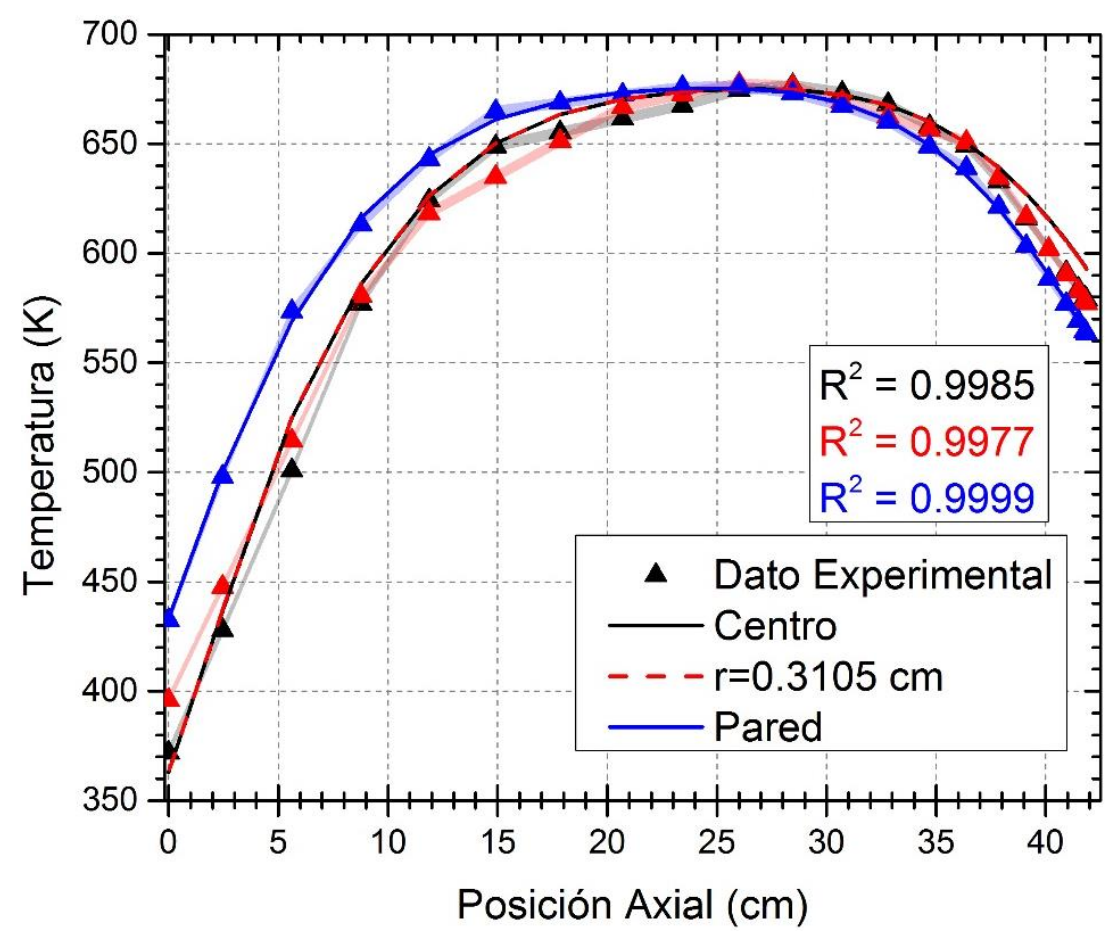

Figura 4.30. Perfil axial de temperatura en el lecho no isotérmico no adiabático con aproximación de la capa límite y empleando el $10 \%$ de $\mathrm{v}_{\mathrm{z}}$ en $\mathrm{v}_{\mathrm{r}}, \operatorname{Re}_{\mathrm{p}}=40.83$,

a 3 posiciones radiales diferentes; ( $\mathbf{\Lambda})$ dato experimental, (-) modelo. 


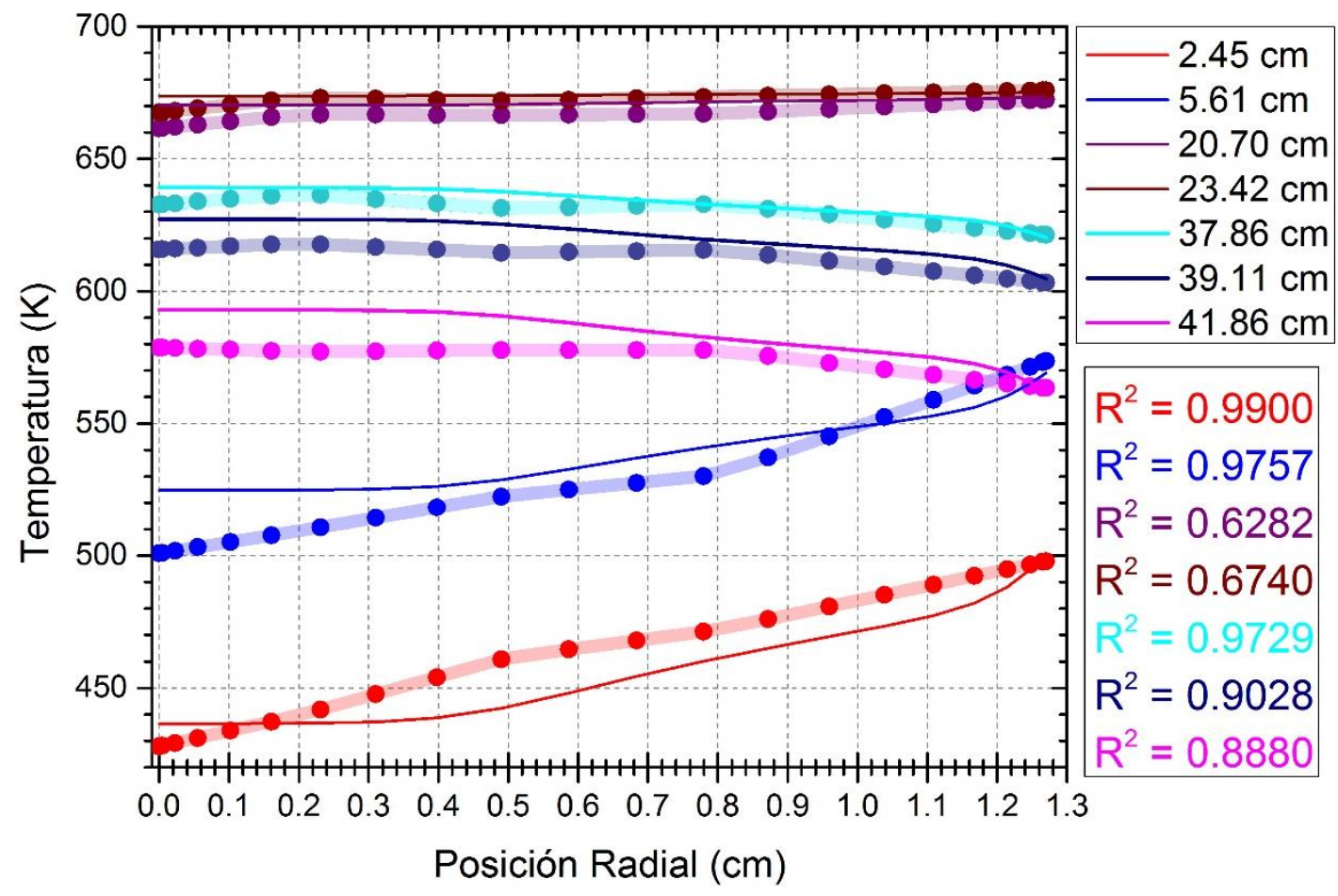

Figura 4.31. Perfil radial de temperatura en el lecho no isotérmico no adiabático con aproximación de la capa límite y empleando el $10 \%$ de $\mathrm{v}_{\mathrm{z}}$ en $\mathrm{v}_{\mathrm{r}}, \mathrm{Re}_{\mathrm{p}}=40.83$,

a 7 posiciones axiales diferentes; ( $\mathbf{\Lambda})$ dato experimental, (-) modelo.

El coeficiente de correlación, $\mathrm{R}^{2}$, de los perfiles de temperatura axial en el centro del lecho y en la pared están por encima del 0.99 , mientras que el $\mathrm{R}^{2}$ en los perfiles radiales de temperatura en la salida del lecho, $\mathrm{z}=41.86 \mathrm{~cm}, 39.11 \mathrm{~cm}$ y $37.86 \mathrm{~cm}$, son de $0.8880,0.9028$ y 0.9729. Es importante señalar que el gradiente de temperatura radial experimental a la salida del lecho es aproximadamente de $15 \mathrm{~K}$, a diferencia del caso donde se tiene un $\operatorname{Re}_{\mathrm{p}}$ de 163.36, cuyo gradiente es de $60 \mathrm{~K}$, lo cual indica que efectivamente existe una pérdida de calor que se incrementa conforme aumenta el flujo del fluido, favorecido por los efectos convectivos de transferencia de calor.

\section{$\operatorname{Re}_{\mathrm{p}}=\mathbf{8 1 . 6 7}$}

Los perfiles de temperatura axiales y radiales del lecho con calentamiento de pared a un Rep de 81.67 se muestran en las figuras 4.32 y 4.33 . El valor estimado de la $k_{\text {eff,r }}$ es de 0.4758 $\mathrm{W} / \mathrm{m} \mathrm{K}$, y sus I.C. del $95 \%$ son $0.4757-0.4759$. Nuevamente se observa que el punto caliente se va desplazando hacia la salida del lecho conforme aumenta el flujo del fluido. 


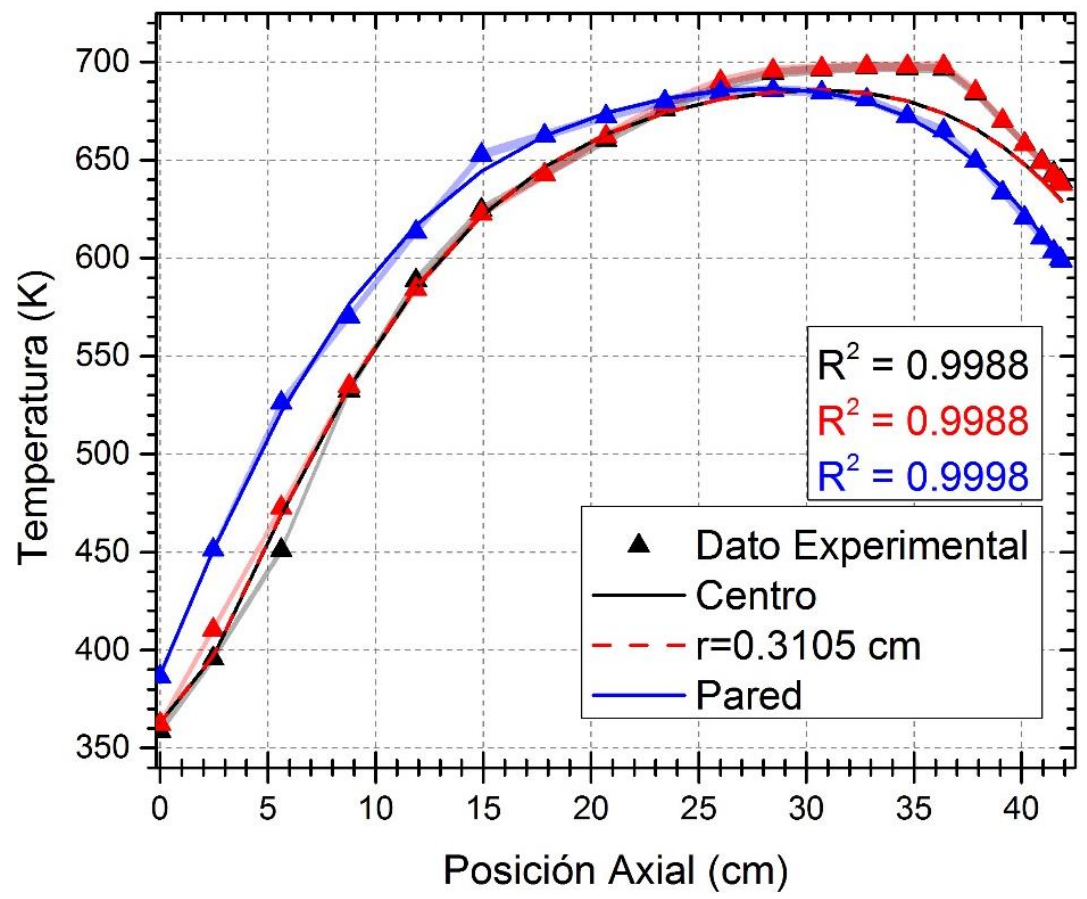

Figura 4.32. Perfil axial de temperatura en el lecho no isotérmico no adiabático con aproximación de la capa límite y empleando el $10 \%$ de $v_{z}$ en $v_{r}, R_{p}=81.67$,

a 3 posiciones radiales diferentes; ( $\mathbf{\Delta})$ dato experimental, (-) modelo.

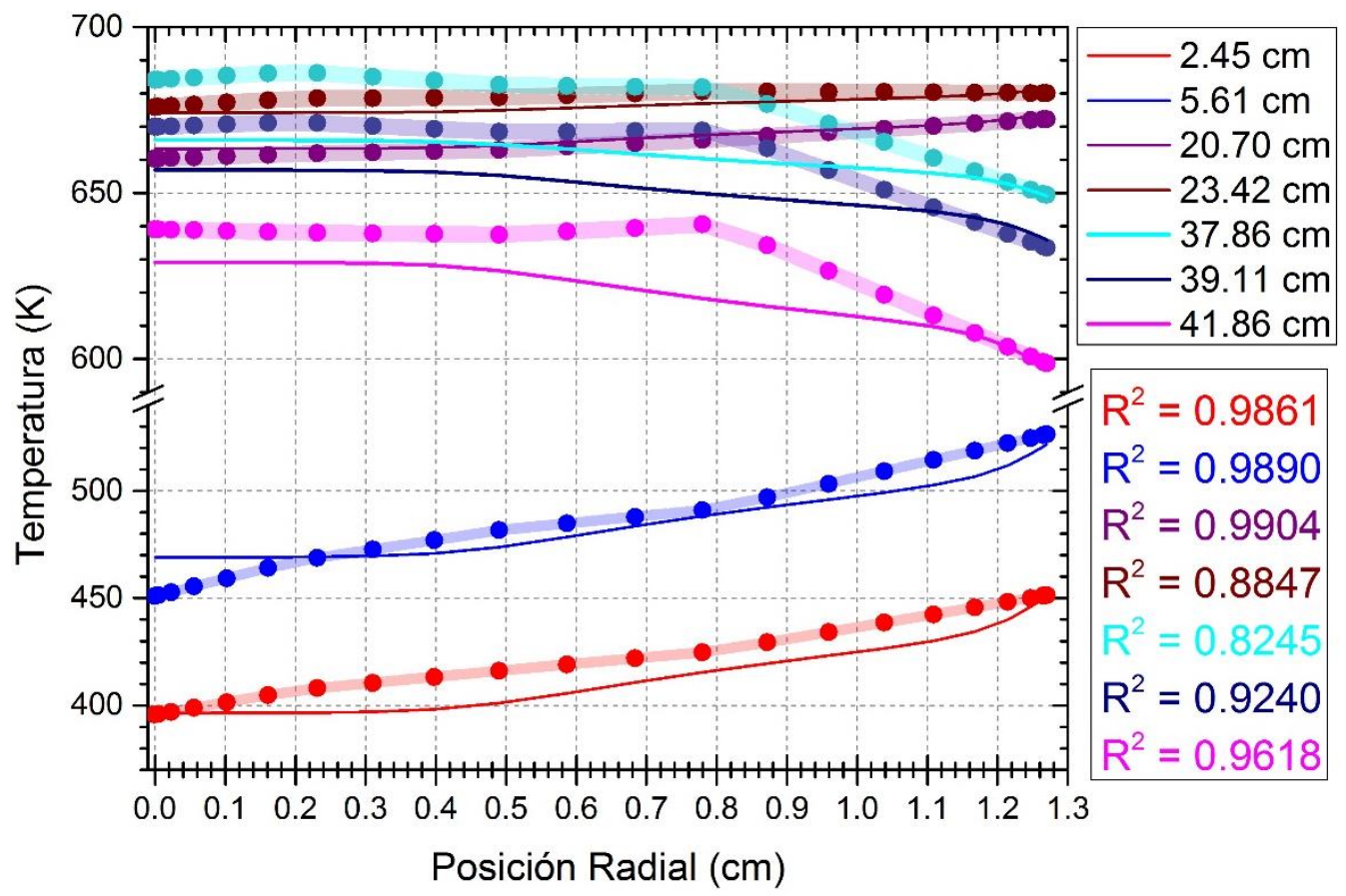

Figura 4.33. Perfil radial de temperatura en el lecho no isotérmico no adiabático con aproximación de la capa límite y empleando el $10 \%$ de $v_{z}$ en $v_{r}, \operatorname{Re}_{p}=40.83$,

a 7 posiciones axiales diferentes; ( $\mathbf{\Delta})$ dato experimental, (-) modelo. 
El coeficiente de correlación, $\mathrm{R}^{2}$, de los perfiles de temperatura axial en el centro del lecho y en la pared están por encima del 0.99 , mientras que el $\mathrm{R}^{2}$ en los perfiles radiales de temperatura en la salida del lecho, $\mathrm{z}=41.86 \mathrm{~cm}, 39.11 \mathrm{~cm}$ y $37.86 \mathrm{~cm}$, son de $0.9618,0.9240$ y 0.8245 , en donde el gradiente de temperatura radial experimental a la salida del lecho es aproximadamente de $40 \mathrm{~K}$.

\subsubsection{Correlación de keff,r}

Con la información obtenida de la caracterización de la transferencia de calor a tres $\operatorname{Re}_{\mathrm{p}}$ en el lecho con calentamiento en la pared, se puede obtener una correlación para el valor de la $\mathrm{k}_{\mathrm{eff}, \mathrm{r}}$ en este sistema con la configuración geométrica presentada en el capítulo 3 , y sus respectivas propiedades físicas.

Teniendo un coeficiente de correlación de 1.00 entre los datos obtenidos por la correlación propuesta y las $\mathrm{k}_{\mathrm{eff}, \mathrm{r}}$ estimadas para los $\mathrm{Re}_{\mathrm{p}}$ empleados en este trabajo, a continuación se presenta dicha correlación

$$
k_{e f f, r}=0.1424+\delta \operatorname{PrR} e_{p}
$$

En donde $\delta$ tiene un valor de 0.005556 , y sus I.C. del 95\% son $0.005504-0.005608$. Cabe señalar que las correlaciones de la literatura presentas en la Figura 2.4 tienen una diferencia entre el $16 \%$ y el $84.5 \%$ con la correlación propuesta en este trabajo. 


\section{Capítulo V-Conclusiones}

Las conclusiones principales de este trabajo se resumen a continuación:

- La contribución sólida (estática) tiene un efecto importante en la caracterización de la transferencia de calor del lecho empacado quasi-adiabático y con calentamiento en la pared, esencialmente cuando este opera en estado transitorio.

- La hidrodinámica, considerando la componente de velocidad axial y/o radial, influye significativamente en los mecanismos radiales de transferencia de calor.

- No es adecuado el uso de correlaciones de la literatura para emplearlas en modelos que describan los mecanismos de transferencia de calor del sistema de estudio, por lo que es necesario el desarrollo de correlaciones propias del sistema, las cuales evalúan los parámetros de transferencia de calor tomando en cuenta las contribuciones estática y dinámica.

- A pesar de que existan distintas aproximaciones que caracterizan parcialmente de manera adecuada la transferencia de calor en un lecho con calentamiento en la pared, existe incertidumbre en el valor empleado de la $\mathrm{k}_{\mathrm{eff}, \mathrm{r}}$. En este trabajo se evaluaron siete aproximaciones de la transferencia de calor en un lecho empacado con calentamiento en la pared, en donde los resultados son parcialmente favorables. El valor de $\mathrm{k}_{\text {eff,r }}$ de cada aproximación varía entre un $8.11 \%$ y un $42.13 \%$ de los otros valores estimados de $\mathrm{k}_{\mathrm{eff}, \mathrm{r}}$. Lo anterior hace notar que, actualmente, no existe un acuerdo de qué modelo describe mejor fenomenológicamente los mecanismos de transferencia de calor en un lecho empacado.

Conclusiones más específicas se muestran a continuación para el lecho quasi-adiabático y el lecho con calentamiento en la pared.

Las conclusiones obtenidas del lecho quasi-adiabático son las siguientes:

- El modelo adiabático pseudo-homogéneo en estado transitorio describe satisfactoriamente los datos observados en el estado estacionario del lecho quasiadiabático; $\mathrm{R}^{2}$ mayores a 0.99 . No obstante, es necesario el desarrollo de correlaciones propias del sistema de estudio, debido a la diversidad de correlaciones que existen en la literatura y a la sensibilidad de los parámetros efectivos en la 
descripción de los perfiles de temperatura en las condiciones de operación que se emplea.

- El modelo adiabático pseudo-heterogéneo en estado transitorio describe satisfactoriamente los datos observados en el estado estacionario del lecho quasiadiabático; $\mathrm{R}^{2}$ mayores a 0.99 . Este modelo no muestra una diferencia de temperatura apreciable con respecto al modelo adiabático pseudo-homogéneo en estado transitorio, lo anterior debido a que al considerar el sistema como un pseudocontinuo, los mecanismos dependientes e independientes del fluido son capturados en este parámetro sin importar la fase utilizada. También es observado que el parámetro $h_{g}$ no tiene un efecto apreciable para describir el estado estacionario del lecho, debido a que no existe un gradiente de temperatura apreciable entre ambas fases, sin embargo, este parámetro juega un papel importante en la transitoriedad de la transferencia de calor.

- El modelo adiabático pseudo-homogéneo en estado transitorio no describe la transitoriedad de la transferencia de calor en el lecho; $R^{2}$ entre 0.84 y 0.96 . Lo anterior es debido a que a pesar de que si describe el estado estacionario, se llega a este en un tiempo rápido a comparación de los datos observados en la transitoriedad. Se cree que al utilizar solamente un parámetro que captura todos los mecanismos de transferencia de calor, este no puede describir por sí solo la transitoriedad. También se comprueba que no existe una diferencia apreciable en la estimación de keff,z en estado transitorio o estacionario, debido a que este parámetro captura los mecanismos de transferencia de calor que describen el gradiente de temperatura en el estado estacionario.

- El modelo adiabático pseudo-heterogéneo en estado transitorio no describe la transitoriedad de la transferencia de calor en el lecho, $\mathrm{R}^{2}$ entre 0.81 y 0.97 . Lo anterior es debido a que ambas fases, sólido y fluido, llegan a un estado estacionario en un tiempo menor al observado experimentalmente. Sin embargo, se hipotetiza, mediante un análisis de la capacidad térmica de la fase sólida, $\mathrm{Cp}_{\mathrm{s}}$, que el uso de la $\mathrm{Cp}_{\mathrm{s}}$, la cual fue estimada para una sola partícula, no es adecuado para el modelo pseudoheterogéneo, debido a que se considera la fase sólida como un esqueleto hecho de partículas, en donde las fracciones vacías juegan un papel importante debido al fluido 
que se encuentra en estas, en donde la cantidad de calor necesaria para elevar un grado centígrado un gramo del esqueleto aumenta. Tomando en cuenta lo anterior, se cree que la descripción de la transitoriedad en un lecho empacado es posible, considerando un mecanismo de transporte convectivo, la contribución del sólido y un gradiente de temperatura entre ambas fases en la transitoriedad para la estimación confiable del parámetro $h_{g}$.

Las conclusiones obtenidas del lecho con calentamiento en la pared son las siguientes:

- Los modelos no isotérmico y no adiabático en estado transitorio, considerando el uso de un flujo pistón o la hidrodinámica, en su componente de velocidad axial, los parámetros $\mathrm{k}_{\mathrm{eff}, \mathrm{r}} \mathrm{y} \mathrm{h}_{\mathrm{w}}$, y tomando en cuenta todas las observaciones para la estimación de parámetros, describen parcialmente los perfiles de temperatura en la mayor parte de la longitud del lecho. Sin embargo, ambos modelos son incapaces de describir la salida del lecho, principalmente en la región central. Lo anterior es debido a que no se cuenta con una medición del calor perdido en la salida del lecho. Además, en la estimación de los parámetros $\mathrm{k}_{\mathrm{eff}, \mathrm{r}} \mathrm{y} \mathrm{h}_{\mathrm{w}}$, existe una alta correlación estadística.

- El modelo no isotérmico y no adiabático en estado transitorio, considerando el uso de la hidrodinámica en la componente de velocidad axial, la aproximación de la capa límite, y tomando en cuenta todas las observaciones para la estimación de parámetros, describe parcialmente de manera adecuada los perfiles de temperatura en la mayor parte de la longitud del lecho. Sin embargo, el modelo no describe la salida del lecho, principalmente en la región central del lecho, debido a una pérdida de calor no tomada en cuenta en la salida del lecho. Cabe señalar que este modelo no cuenta con una correlación estadística, debido a que sólo la $\mathrm{k}_{\mathrm{eff}, \mathrm{r}}$ es utilizada para describir los mecanismos de transferencia de calor radiales.

- El uso de los modelos no isotérmico y no adiabático en estado transitorio, considerando el uso de la hidrodinámica en la componente de velocidad axial, la aproximación de la capa límite, una velocidad radial del 10\%, 15\% y 20\%, y tomando en cuenta todas las observaciones para la estimación de parámetros, describen parcialmente de manera adecuada los perfiles de temperatura en la mayor parte de la longitud del lecho. Sin embargo, el no contar con una pérdida de calor en la condición 
de frontera a la salida del lecho, deteriora la caracterización de transferencia de calor en el lecho. El uso de una $v_{r}$ muestra un aumento en la $k_{\text {eff,r }}$ estimada conforme el aumento de $v_{r}$. En estos modelos es evitada una correlación estadística debido a que sólo se hace uso de una keff,r.

- El modelo no isotérmico y no adiabático en estado transitorio, considerando el uso de la hidrodinámica en la componente de velocidad axial, la aproximación de la capa límite, una velocidad radial del 10\%, y tomando en cuenta las observaciones en el centro del lecho para la estimación de parámetros, describe parcialmente de manera adecuada los perfiles de temperatura en la mayor parte de la longitud del lecho. No obstante, la descripción de los mecanismos de transferencia de calor a la salida del lecho son inadecuados debido a la no consideración de una pérdida de calor a la salida del mismo. Además, la descripción en la región de la pared son ligeramente deficientes a aquellos predichos por la keff,r estimada con todas las observaciones del lecho.

- Al comparar las distintas aproximaciones de los modelos no isotérmico y no adiabático en estado transitorio, y la estimación de las k keff,r, se concluyó que todas las aproximaciones dan parcialmente una adecuada descripción de los perfiles de temperatura dentro del lecho empacado con calentamiento en la pared. Sin embargo, la diferencia entre los valores estimados de $\mathrm{k}_{\mathrm{eff}, \mathrm{r}}$ entre estas aproximaciones varían entre un $8.11 \%$ y $42.13 \%$, lo cual genera incertidumbre en la caracterización de los mecanismos de transferencia de calor radial que sucedan dentro del lecho. También se cree que el uso de una keff,r que varíe en función de la posición radial pudiese dar una caracterización adecuada de los mecanismos de transferencia de calor en todo el lecho. Además, el lecho con calentamiento en la pared, al contar con gradientes axiales de temperatura mucho mayores a los estimados en el lecho quasi-adiabático, y gradientes radiales de temperatura diferentes a lo largo del mismo, se cree que el uso de parámetros estimados para un gradiente de temperatura no es adecuado para cuando se tiene otro gradiente con una diferencia notable de temperaturas.

- Se caracterizó parcialmente adecuada la transferencia de calor en el lecho con calentamiento en la pared a dos $\operatorname{Re}_{\mathrm{p}}$ diferentes, 40.83 y 81.67 . Aunado a la anterior, 
se desarrolló una correlación para obtener la keff,r en este lecho con las propiedades físicas y configuración geométrica mostradas en el capítulo 3.

Las propuestas de trabajo a futuro se muestran a continuación:

- Realización de un estudio experimental que sea capaz de medir con certidumbre la temperatura en ambas fases en la transitoriedad para el caso quasi-adiabático y con calentamiento en la pared, así como también más mediciones de temperatura a la salida del lecho, zona en donde se tienen los mayores gradientes de temperatura. También se propone el empleo de un radiómetro que mida la radiación con la que se calienta el lecho con la lámpara de luz infrarroja y sus variaciones cuando exista un cambio de flujo, así como también la lectura de las pérdidas de calor a la salida del lecho cuando se estudie el lecho con calentamiento en la pared. Lo anterior para tener una mayor certidumbre para el este estudio teórico de la transferencia de calor en lechos con relación de $\mathrm{d}_{\mathrm{t}} / \mathrm{d}_{\mathrm{p}}<10$.

- Obtención del $\mathrm{Cp}_{\mathrm{s}}$ del lecho en ausencia de flujos de fluido, y emplearlo un estudio teórico posteriormente.

- Realización de un estudio teórico que evalúe el uso de una keff,r que varíe en función de una posición radial, principalmente para el lecho con calentamiento en la pared, y el empleo de una $\mathrm{Cp}_{\mathrm{s}}$ del esqueleto conformado por las partículas dentro del lecho, esencialmente para la descripción de la transitoriedad del lecho quasi-adiabático y con calentamiento en la pared. 


\section{Referencias bibliográficas}

1. Che-Galicia, G., Ruiz-Martínez, R. S., López-Isunza, F. \& Castillo-Araiza, C. O. Modeling of oxidative dehydrogenation of ethane to ethylene on a $\mathrm{MoVTeNbO} / \mathrm{TiO} 2$ catalyst in an industrial-scale packed bed catalytic reactor. Chem. Eng. J. 280, 682-694 (2015).

2. Castillo-Araiza, C. O. \& López-Isunza, F. Modeling the partial oxidation of o-xylene in an industrial packed-bed catalytic reactor: The role of hydrodynamics and catalyst activity in the heat transport. Ind. Eng. Chem. Res. 49, 6845-6853 (2010).

3. Aparicio-Mauricio, G., Ruiz, R. S., López-isunza, F. \& Castillo-araiza, C. O. A simple approach to describe hydrodynamics and its effect on heat and mass transport in an industrial wall-cooled fixed bed catalytic reactor: ODH of ethane on a MoVNbTeO formulation. Chem. Eng. J. 321, 584-599 (2017).

4. Castillo-Araiza, C. O. Estudio de los Procesos de Transferencia de Calor en un Lecho Empacado. (Universidad Autónoma Metropolitana - Iztapalapa, 2004).

5. Castillo-Araiza, C. O., Jiménez-Islas, H. \& López-Isunza, F. Heat-Transfer Studies in Packed-Bed Catalytic Reactors of Low Tube / Particle Diameter Ratio HeatTransfer Studies in Packed-Bed Catalytic Reactors of Low Tube / Particle Diameter Ratio. Ind. Eng. Chem. Res. 46, 7426-7435 (2007).

6. Özgümüş, T., Mobedi, M., Özkol, Ü. \& Nakayama, A. Thermal Dispersion in Porous Media-A Review on the Experimental Studies for Packed Beds. Appl. Mech. Rev. 65, 31001-1-31001-19 (2013).

7. Kulkarni, B. D. \& Doraiswamy, L. K. Catalysis Reviews : Science and Engineering. Estimation of Effective Transport Properties in Packed Bed Reactors. (1980).

8. Yagi, S. \& Kunii, D. Studies on Effective Thermal Conductivities in Packed Beds. A.I.Ch.E. J. 3, 373-381 (1957).

9. Yagi, S., Kunii, D. \& Wakao, N. Studies on axial effective thermal conductivities in packed beds. AIChE J. 6, 543-546 (1960).

10. Votruba, J., Hlavacek, V. \& Marek, M. Packed bed axial thermal conductivity. Chem. Eng. Sci. 27, 1845-1851 (1972). 
11. Vortmeyer, D. \& Adam, W. Steady-state measurements and analytical correlations of axial effective thermal conductivities in packed beds at low gas flow rates. Int. J. Heat Mass Transf. 27, 1465-1472 (1984).

12. Bunnel, D. G., Irvin, H. B., Olson, R. W. \& Smith, J. M. Effective thermal conductivitiesin gas-solidsystems. Ind. andEngineering Chem. 41, 1977-1981 (1949).

13. Metzger, T., Didierjean, S. \& Maillet, D. Optimal experimental estimation of thermal dispersion coefficients in porous media. Int. J. Heat Mass Transf. 47, 3341-3353 (2004).

14. Elsari, M. \& Hughes, R. Axial effective thermal conductivities of packed beds. Appl. Therm. Eng. 22, 1969-1980 (2002).

15. Wen, D. \& Ding, Y. Heat transfer of gas flow through a packed bed. Chem. Eng. Sci. 61, 3532-3542 (2006).

16. Dixon, A. G. \& Cresswell, D. L. Theoretical prediction of effective heat transport parameters in packed beds. A.I.Ch.E. J. 25, 663-676. (1979).

17. Dixon, A. G. \& van Dongeren, J. H. The influence of the tube and particle diameters at constant ratio on heat transfer in packed beds. Chem. Eng. Process. 37, 23-32 (1998).

18. López-Isunza, F. L. STEADY STATE AND DYNAMIC BEHAVIOUR OF AN INDUSTRIAL FIXED BED CATALYTIC REACTOR. (University of London, 1983).

19. Vortmeyer, D. \& Haidegger, E. Discrimination of three approaches to evaluate heat fluxes for wall-cooled fixed bed chemical reactors. Chem. Eng. Sci. 46, 2651-2660 (1991).

20. Winterberg, M. \& Tsotsas, E. Correlations for effective heat transport coefficients in beds packed with cylindrical particles. Chem. Eng. Sci. 55, 5937-5943 (2000).

21. Winterberg, M., Tsotsas, E., Krischke, A. \& Vortmeyer, D. A simple and coherent set of coeffcients for modelling of heat and mass transport with and without chemical reaction in tubes filled with spheres. Chem. Eng. Sci. 55, 967-979 (2000).

22. Papageorgiou, J. N. \& Froment, G. F. Simulation models accounting for radial 
voidage profiles in fixed-bed reactors. Chem. Eng. Sci. 50, 3043-3056 (1995).

23. Daszkowski, T. \& Eigenberger, G. A reevaluation of fluid flow heat transfer and chemical reaction in catalyst filled tubes. Chem. Eng. Sci. 47 (9-11), 2245-2250 (1992).

24. Borkink, J. G. H. \& Westerterp, K. R. Determination of Effective Heta Transport Coefficients for Wall-Cooled Packed Beds. Chem. Eng. Sci. 47, 2337-2342 (1992).

25. Borkink, J. G. H., Borman, P. C. \& Westerterp†, K. R. Modelling of Radial Heat Transport in Wall-Cooled Packed Beds Confidence Intervals of Estimated Parameters and Choice of Boundary Conditions. Chem. Eng. Commun. 121, 135155 (1993).

26. Michelsen, M. L. Estimation of heat transfer parameters in packed beds from radial temperature measurements. Chem. Eng. J. 18, 67-72 (1979).

27. Tsang, T. H., Edgar, T. F. \& Hougen, J. O. Estimation of Heat Transfer Parameters in a Packed Bed. Chem. Eng. J. 11, 57-66 (1976).

28. De Wasch, A. P. \& Froment, G. F. Heat transfer in packed beds. Chem. Eng. Sci. 27, 567-576 (1972).

29. Fernández, J. R., Alarcón, J. M. \& Abanades, J. C. Investigation of a Fixed-Bed Reactor for the Calcination of $\mathrm{CaCO} 3$ by the Simultaneous Reduction of $\mathrm{CuO}$ with a Fuel Gas. Ind. Eng. Chem. Res. 55, 5128-5132 (2016).

30. Wan, Z. et al. A study of the heat transfer characteristics of novel Ni-foam structured catalysts. Can. J. Chem. Eng. 94, 2225-2234 (2016).

31. Jorge, L. M. de M., Jorge, R. M. M. \& Giudici, R. Experimental and numerical investigation of dynamic heat transfer parameters in packed bed. Heat Mass Transf. 46, 1355-1365 (2010).

32. Anderson, R., Shiri, S., Bindra, H. \& Morris, J. F. Experimental results and modeling of energy storage and recovery in a packed bed of alumina particles. Appl. Energy 119, 521-529 (2014).

33. Paterson, W. R. \& Carberry, J. J. Fixed bed catalytic reactor modelling: The heat transfer problem. Chem. Eng. Sci. 38, 175-180 (1983).

34. Thoméo, J. C. \& Freire, J. T. Heat transfer in fixed bed: a model non-linearity 
approach. Chem. Eng. Sci. 55, 2329-2338 (2000).

35. Dixon, A. G. \& Cresswell, D. L. Effective heat transfer parameters for transient packed-bed models. AIChE J. 32, 809-819 (1986).

36. Dixon, A. G. The length effect on packed bed effective heat transfer parameters. Chem. Eng. J. 31, 163-173 (1985).

37. Smirnov, E. I., Kuzmin, V. a. \& Zolotarskii, I. a. Radial Thermal Conductivity in Cylindrical Beds Packed by Shaped Particles. Chem. Eng. Res. Des. 82, 293-296 (2004).

38. Foumeny, E. A. \& Ma, J. Non-Darcian non-isothermal compressible flow and heat transfer in cylindrical packed beds. Chem. Eng. Technol. 17, 50-60 (1994).

39. Bey, O. \& Eigenberger, G. Gas flow and heat transfer through catalyst filled tubes. Int. J. Therm. Sci. 40, 152-164 (2001).

40. McGreavy, C., Foumeny, E. A. \& Javed, K. H. Characterization of transport properties for fixed bed in terms of local bed structure and flow distribution. Chem. Eng. Sci. 41, 787-797 (1986).

41. Dixon, A. G. Fixed bed catalytic reactor modelling-the radial heat transfer problem. Can. J. Chem. Eng. 90, 507-527 (2012).

42. Behnam, M., Dixon, A. G., Nijemeisland, M. \& Stitt, E. H. A New Approach to Fixed Bed Radial Heat Transfer Modeling Using Velocity Fields from Computational Fluid Dynamics Simulations BT - Industrial \& Engineering Chemistry Research. Ind. Eng. Chem. Res. 52, 15244-15261 (2013).

43. Smirnov, E. I., Muzykantov, A. V., Kuzmin, V. A., Kronberg, A. E. \& Zolotarskii, I. A. Radial heat transfer in packed beds of spheres, cylinders and Rashig rings Verification of model with a linear variation of ker in the vicinity of the wall. Chem. Eng. J. 91, 243-248 (2003).

44. Dekhtyar, R. A., Sikovsky, D. P., Gorine, A. V. \& Mukhin, V. A. Heat transfer in a packed bed at moderate values of the Reynolds number. High Temp. 40, 693-700 (2002).

45. SPECCHIA, V., BALDI, G. \& SICARDI, S. Heat Transfer in Packed Bed Reactors With One Phase Flow. Chem. Eng. Commun. 4, 361-380 (1980). 
46. Yagi, S. \& Wakao, N. Heat and Mass Transfer from Wall to Fluid in Packed Beds. AIChE J. 5, 79-85 (1959).

47. Gunn, D. J. \& KHALID, M. Thermal Dispersion and Wall Thermal Dispersion And Wall Heat Transfer In Packed Beds. Chem. Eng. Sci. 30, 261-267 (1975).

48. Schertz, W. W. \& Bischoff, K. B. Thermal and material transport in nonisothermal packed beds. AIChE J. 15, 597-604 (1969).

49. Marivoet, J., Teodoroiu, P. \& Wajc, S. J. Porosity, velocity and temperature profiles in cylindrical packed beds. Chem. Eng. Sci. 29, 1836-1840 (1974).

50. Lerou, J. J. \& Froment, G. F. Velocity, temperature and conversion profiles in fixed bed catalytic reactors. Chem. Eng. Sci. 32, 853-861 (1977).

51. Clement, K. \& Jorgensen, B. Experimental Investigation Of Axial And Radial Thermal Dispersion In A Packed Bed. Chem. Eng. Sci. 38(6), 835-842 (1983).

52. Borkink, J. G. \& Westerterp, K. R. Influence of tube and particle diameter on heat transport in packed beds. AIChE J. 38, 703-716 (1992).

53. Nie, X. D., Besant, R. W. \& Evitts, R. W. Heat Transfer Between Gas-Solid Phases Within Packed Particle Beds. Part. Sci. Technol. 29, 151-162 (2011).

54. Cong, T. N., He, Y., Chen, H., Ding, Y. \& Wen, D. Heat transfer of gas-solid twophase mixtures flowing through a packed bed under constant wall heat flux conditions. Chem. Eng. J. 130, 1-10 (2007).

55. Polesek-Karczewska, S. Effective thermal conductivity of packed beds of spheres in transient heat transfer. Heat Mass Transf. 39, 375-380 (2003).

56. Schröder, E., Class, A. \& Krebs, L. Measurements of heat transfer between particles and gas in packed beds at low to medium Reynolds numbers. Exp. Therm. Fluid Sci. 30, 545-558 (2006).

57. Laguerre, O., Ben Amara, S., Alvarez, G. \& Flick, D. Transient heat transfer by free convection in a packed bed of spheres: Comparison between two modelling approaches and experimental results. Appl. Therm. Eng. 28, 14-24 (2008).

58. Finlayson, B. A. Packed bed reactor analysis by orthogonal collocation. Chem. Eng. Sci. 26, 1081-1091 (1971). 
59. Delmas, H. \& Froment, G. F. A simulation model accounting for structural radial nonuniformities in fixed bed reactors. Chem. Eng. Sci. 43, 2281-2287 (1988).

60. Ströhle, S., Haselbacher, A., Jovanovic, Z. R. \& Steinfeld, A. Transient discretegranule packed-bed reactor model for thermochemical energy storage. Chem. Eng. Sci. 117, 465-478 (2014).

61. Castillo-Araiza, C. O. Estudio del comportamiento del reactor de oxidación parcial de o-xileno a anhídrido ftálico. Universidad Autónoma Metropolitana (2008).

62. Wakao, N., Kaguei, S. \& Funazkri, T. Effect of fluid dispersion coefficients on particle-to-fluid heat transfer coefficients in packed beds. Chem. Eng. Sci. 34, 325336 (1979). 


\section{Nomenclatura}

\section{Letras latinas}
$\mathrm{a}_{\mathrm{s}} \quad$ Área específica
$\mathrm{C}_{\mathrm{p}} \quad$ Calor específico, $\mathrm{J} / \mathrm{g} \mathrm{K}$
$\mathrm{d}_{\mathrm{p}} \quad$ Diámetro de partícula, $\mathrm{m}$
$\mathrm{d}_{\mathrm{t}} \quad$ Diámetro de tubo, $\mathrm{m}$
F Función objetivo
G Flux másico, $\mathrm{kg} / \mathrm{m}^{2} \mathrm{~s}$
$\mathrm{g}_{\mathrm{z}} \quad$ Componente axial de la gravedad, $\mathrm{m} / \mathrm{s}^{2}$
$\mathrm{h}_{\mathrm{g}} \quad$ Coeficiente efectivo de transporte de calor interfacial, $\mathrm{W} / \mathrm{m}^{2} \mathrm{~K}$
$\mathrm{h}_{\mathrm{w}} \quad$ Coeficiente efectivo de transporte de calor en la pared, $\mathrm{W} / \mathrm{m}^{2} \mathrm{~K}$
K Permeabilidad del lecho empacado
$\mathrm{k}_{\mathrm{eff}} \quad$ Conductividad térmica efectiva, $\mathrm{W} / \mathrm{m} \mathrm{K}$
$\mathrm{k}_{\mathrm{f}} \quad$ Conductividad térmica del fluido, W/m K
ks Conductividad térmica del sólido, W/m K
$\mathrm{K}_{\mathrm{z}} \quad$ Pseudo-permeabilidad del lecho empacado
L Longitud del lecho empacado, $\mathrm{m}$
$\mathrm{p}_{\mathrm{z}} \quad$ Componente axial de la presión, $\mathrm{kg} / \mathrm{m} \mathrm{h}^{2}$
q Flux de calor, $\mathrm{W} / \mathrm{m}^{2}$
r Posición radial, $m$
$\mathrm{Re}_{\mathrm{p}} \quad$ Número de Reynolds de partícula, $\mathrm{m}$
$\mathrm{t}$ Tiempo, $\mathrm{s}$
T Temperatura, $\mathrm{K}$
$\mathrm{U}$ Coeficiente global de transferencia de calor, $\mathrm{W} / \mathrm{m}^{2} \mathrm{~K}$
$\mathrm{v}$ velocidad, $\mathrm{m} / \mathrm{s}$
z Posición axial, m 


\section{Letras griegas}

$\alpha \quad$ Parámetro que captura los efectos hidrodinámicos de la $\mathrm{k}_{\mathrm{eff}, \mathrm{z}}$

$\beta \quad$ Parámetro que captura los efectos hidrodinámicos de la keff,z

$\delta \quad$ Parámetro que captura los efectos hidrodinámicos de la keff,r

$\varepsilon \quad$ Fracción vacía

$\rho \quad$ Densidad, $\mathrm{kg} / \mathrm{m}^{3}$

$\mu_{\text {eff }} \quad$ Viscosidad efectiva, Pa s

\section{Subíndices}

0 Referencia a una condición inicial

b Referencia al baño que calienta externamente el lecho

exp Referencia a mediciones experimentales

f Referencia a la fase fluida

in Referencia a la entrada del lecho

mod Referencia a predicciones del modelo

S Referencia a la fase sólida

ss Referencia al estado estacionario

t Referencia a la pared del tubo

W Referencia a la pared del lecho

z Referencia a la coordenada axial

\section{Superíndices}

0 Referencia a la ausencia de flujo de un fluido

CR Referencia a la zona central del lecho empacado

WR Referencia a la zona de la pared del lecho empacado 